\title{
Questioning the orbital picture of magnetic spin coupling: a real space alternative
}

\author{
A. Martín Pendás* and E. Francisco \\ Departamento de Química Física y Analítica, Facultad de Química, Universidad de Oviedo, \\ E-33006-Oviedo, Spain. \\ E-mail: ampendas@uniovi.es
}

\begin{abstract}
The prevailing magnetic spin coupling paradigm is based on a one-electron picture, thus being orbital dependent and unsatisfactory from a physical point of view. We examine it under a truly invariant real space perspective, focusing on the role of electron delocalization. We show that this view, compatible with orbital thinking, overcomes its limitations. By examining simple model systems we show that it is electron delocalization that drives any singlet-triplet gap, and that delocalization and ionic mixing are two sides of the same reality. It is in the end delocalization, fostered or hindered by the specific structure of a system, that lies behind its preferred magnetic coupling mode. In the case of superexchange-mediated coupling through atomic bridges, we also point out the non-essential role of the bridge's electrons in setting up singlet-triplet preferences. We show that the use of real space thinking allows for tuning singlet-triplet gaps using knobs that are not easily grasped from the orbital standpoing, opening new avenues in the design and control of molecular magnets.
\end{abstract}




\section{Introduction}

The fascination of humankind by magnets has accompanied our species for millennia, ${ }^{1}$ although it took a considerable amount of time and ingenuity until Faraday and Maxwell's systematizations led to the electromagnetic field equations. The discovery of the electron and its consecration at the International Congress of Physics held in Paris in 1900 laid the grounds to solve the problem of the sources of the fields, but opened others. Langevin, for instance, noted that paramagnetism required the existence of atoms with permanent magnetic moment, but it was not until Goudsmit and Uhlenbeck ${ }^{2}$ demonstrated the existence of an intrinsic magnetic moment for the electron and Dirac combined Einstein's relativity with quantum mechanics, ${ }^{3}$ that the electron's spin was finally understood.

The rapid success of quantum theory in providing a successful explanation of magnetic phenomena was impressive, and by the early 1930's two influential books on the subject had already been written by Van Vleck $(1932)^{4}$ and Stoner (1934). ${ }^{5}$ Since then, the theory of magnetic couplings has been intimately linked to the concept of exchange, ${ }^{6}$ and to various phenomenological models like those of Heisenberg, Dirac, and Van Vleck, ${ }^{4,6,7}$ or Ising. ${ }^{8}$ In the former, for instance, an effective spin Hamiltonian $H=-\sum_{i j} J_{i j} \boldsymbol{S}_{i} \cdot \boldsymbol{S}_{j}$ is written in terms of intercenter magnetic exchange coupling constants $J_{i j}$, from which collective behaviors can be simulated and macroscopic susceptibilities computed. The J's have been traditionally fitted against experimental results, and soon efforts were made to compute them ab initio. Historically (an authorized account can be found in the book by Mattis ${ }^{1}$ ), this problem was initially tackled with the Heitler-London (HL) methodology, which coined much of the language still used today, but the inherent difficulties of using non-orthogonal one-electron functions in the incipient valence-bond (VB) theory led to catastrophic simplifications in dealing with any but the simplest systems. This led Slater, ${ }^{9}$ among others, to advocate for using orthogonal (Wannier) functions and ultimately to the adoption of the method of Mulliken and co-workers which resulted in molecular orbital (MO) theory.

In the simplest case of the bare coupling between two spin $1 / 2$ centers, $a$ and $b$, the 
HL method predicts the correct singlet ground state when using just two localized nonorthogonal functions $\left(\varphi_{a}, \varphi_{b}\right)$, and has provided chemists with a set of primary tools with which to rationalize and control the magnetic interactions in a wealth of transition metal or organic radical compounds. ${ }^{10}$ In the weak overlap limit, the HL machinery leads to the KahnBriat $(\mathrm{KB})$ model, ${ }^{11}$ with a singlet-triplet splitting $\Delta_{S T}=E_{S}-E_{T} \approx 2 K_{a b}+4 \beta S_{a b}$, where $K_{a b}$ is the bare exchange integral between the $a, b$ functions, $\beta$ is the (negative) non-diagonal one-electron hamiltonian matrix element $\left\langle\varphi_{a}|h| \varphi_{b}\right\rangle$, and $S_{a b}$ is the overlap integral $\left\langle\varphi_{a} \mid \varphi_{b}\right\rangle$. The use of localized orthogonal orbitals in the HL ansatz (the so-called Coulson-Fischer orbitals), gives rise to an unbound singlet, and to a triplet ground state, with $\Delta_{S T}=2 K_{a b}$. Slater himself ${ }^{9}$ showed that this catastrophic result can be remedied by mixing the covalent and ionic HL wavefunctions, introducing the idea that it is the larger variational flexibility of the singlet which stabilizes it below the triplet.

Meanwhile, the antiferromagnetic spin pattern revealed by neutron diffraction in $\mathrm{MnO}^{12}$ led Anderson ${ }^{13}$ to explain the inconceivably large singlet coupling among the $\mathrm{Mn}^{2+}$ ions in terms of a modified version of Kramers' ${ }^{\prime 14}$ mediated spin-coupling theory. This superexchange mechanism is essentially a through-bond (instead of a through-space) coupling which is more efficient for singlets due to their larger variational space or, in more chemical terms, due to the presence of exchange pathways in the singlet that include ionic structures involving the bridging ligands, absent in the triplet. After several modifications, advanced by Goodenough, Kanamori, Anderson, and many others, ${ }^{15,16}$ Anderson's superexchange became the standard interpretive model of magnetic coupling through barriers for physicists.

In the chemical literature, the MO equivalent to the Kahn-Briat model appeared in 1975 in a seminal paper by Hay, Thibeault, and Hoffmann ${ }^{17}(\mathrm{HTH})$. In its simplest version the pair of singly occupied orbitals obtained for the triplet state (those involved in the magnetic coupling), $\phi_{1}, \phi_{2}$, are subjected to a localizing unitary rotation to give two orthogonal localized magnetic orbitals (OLOs), $\phi_{a}, \phi_{b}$. The variational space of this effective two-orbital, two-electron system contains two singlets and one triplet (being thus larger for the singlet). 
Diagonalization in this $2 \times 2$ space followed by approximations equivalent to those of the Kahn-Briat model leads to $\Delta E_{S T}=2 K_{a b}-\left(\epsilon_{1}-\epsilon_{2}\right)^{2} /\left(J_{a a}-J_{a b}\right)$, where $K_{a b}$ is the bare exchange integral between the OLOs, $\epsilon_{1}, \epsilon_{2}$ the orbital energies of the triplet state MOs, and $J_{a a}, J_{a b}$ the onsite and inter-site Coulomb repulsions between the OLO's. A simple change of notation transforms the HTH expression into $\Delta E_{S T}=2 K_{a b}-4 t_{a b}^{2} / U$, where $t_{a b}=\left\langle\phi_{a} \bar{\phi}_{b}|H| \phi_{a} \bar{\phi}_{a}\right\rangle$ is the transfer integral and $U$ Hubbard's on-site repulsion. It is not difficult to show with the help of quasi-degenerate perturbation theory ${ }^{18}$ that the presence of a closed-shell bridge (described by bridge localized orbitals) between the magnetic centers provides an equivalent $\Delta E_{S T}$ expression with an enhanced effective, or dressed, $t_{a b}^{e f f}$ element. This provides the MO basis for superexchange pathways.

Over the years, these ideas have crystallized in computationally efficient procedures that use large active spaces to capture the orbital quasi-degeneracies of the multi-electron states of transition metal ions, and in specific perturbation techniques, like the very successful difference dedicated configuration interaction method (DDCI) of Malrieu and co-workers, ${ }^{19}$ to deal with the multitude of exchange pathways and the subtleties of dynamic correlation. State of the art calculations are now precise to the $\mathrm{cm}^{-1}$, representing accuracies of one part in $10^{7},{ }^{20}$ and DFT methods, using broken symmetry approaches, have also been extremely successful. ${ }^{21}$ In most cases, calculations are interpreted by mapping back the results of the accurate computations onto HTH-like qualitative valence-only models that allegedly capture the essential physics of the problem. ${ }^{22,23}$

The simplicity and ability of the HTH or KB models to rationalize the magnetic behavior of simple systems has forged several generations of chemists and physicists. The small, everpresent ferromagnetic direct exchange contributions are usually outweighed by generally mediated antiferromagnetic terms, which can be tuned by modulating the overlap integrals in the KB model or the transfer and on-site terms in the HTH expression. This has been also the basis for understanding magnetostructural correlations. For instance, the vast literature on dinuclear $3 d$ complexes $^{10}$ shows a subtle dependence of $J$ with the bridging ligand (L) 
and the $\widehat{\mathrm{MLM}}$ angle ( $\mathrm{M}$ is the metal). The rather rare ferromagnetic ground states are found near the allowed crossings of the $\epsilon_{1}$ and $\epsilon_{2}$ functions, i.e. when the KB overlap term $4 \beta S_{a b}$ is zero or negligible and the positive $K_{a b}$ prevails. When simple atoms act as bridging species, it is easily seen that such a crossing lies when the $\widehat{\mathrm{MBM}}$ angle is close to $90^{\circ}$, in agreement with the experimental evidence. ${ }^{10}$

Despite the success of this simple orbital paradigm, its roots are conceptually unsatisfactory from several independent fronts, even when taking the dihydrogen molecule as the simplet possible model system. On one hand, if a non-orthogonal, HL, descriptions is used, the ground state is correctly assigned as a singlet at all internuclear separations. Partitioning $J$ into ferromagnetic and antiferromagnetic contributions is here unjustified, for the $K_{a b}$ integral is always smaller in magnitude than the other negative terms. On the other hand, if an orthogonal picture is assumed, the HL functions built with Coulson-Fischer orbitals leads to a catastrophic triplet unbound ground state. Finally, if a general orthogonal MO framework is adopted, a minimal configuration interaction mixing the two closed-shell singlet determinants is needed. Interpreting the results now requires an orbital localization step which provides the basic elements of the HTH machinery. Aside from cases where symmetry fixes the answer, the localization can be performed in infinitely many ways, and different values for the ferro- and antiferro- components of $\Delta_{S T}$ will be obtained thereafter.

In other words, since the prevailing magnetic spin coupling paradigm is based on a oneelectron picture, it is orbital dependent. Any satisfactory explanation of the physics of magnetic coupling should be orbital invariant, a requisite that can be fulfilled by examining the problem in real space, as we advocate in the present work. As a simple example, it has been written many times that the HL unbound singlet built from Coulson-Fischer orbitals becomes stabilized in dihydrogen thanks to the mixing with ionic structures. ${ }^{24}$ This is also, as already noted, the basis of the superexchange enhancement of $J$. However, the standard non-orthogonal HL singlet is a rather accurate approximation to the bound $\mathrm{H}_{2}$ ground state, while VB-oriented contemporary researchers ${ }^{25}$ would surely agree that it does not contain 
any ionic mixing. If a real space perspective is adopted, then it is clear that in all these cases a certain amount of ionic mixing exists, since when the tail of an a localized orbital approaches the $b$ nucleus the probability of finding the two electrons in the $b$ atom (an ionic contribution) is not zero. ${ }^{26}$ Since the amount of ionic structures is easily found to be smaller (vide infra) in the Coulson-Fischer HL function, the argument of singlet stabilization by ionic mixing does actually work. In fact, as we are showing, there is no bonding and no singlet-triplet splitting without ionic mixing whatsoever.

It is our aim in this manuscript to offer a consistent real space story of magnetic spin coupling in the simplest possible model systems. As it will be seen, we have selected them to show our arguments with clarity, but our conclusions are of general validity. We will start by showing that electron delocalization is a necessary requisite for binding to occur, and that without it there can be no singlet-triplet splitting. When an orbital-free perspective is adopted, the $K_{a b}$ ferromagnetic direct exchange gives way to antisymmetry based arguments that flow into the electron-electron repulsion energy components. We also show that angular dependent singlet-triplet switching occurs in the simplest $\mathrm{H}-\mathrm{He}-\mathrm{H}$ chain, even with minimal basis sets, when the overlap logic cannot simply work. This switching is present even when the atomic bridge is substituted by a one-electron pseudopotential, a finding that casts doubts on the true necessity of the superexchange mechanism. A real space perspective is then found to offer an orbital invariant view compatible with the basics of the HTH scheme, but independent of it. To that end, we apply a battery of real space techniques, using a quantum theory of atoms in molecules (QTAIM) atomic partition, ${ }^{27}$ the interacting quantum atoms (IQA) energy decomposition, ${ }^{28,29}$ real space resonance structures from the theory of electron distribution functions (EDFs), ${ }^{30,31}$ and an open systems compatible local spin decomposition, ${ }^{32}$ among others. Altogether, these methods build a narrative in which antisymmetry requirements justify the success of orbital models, but overcome their limitations. We clearly show that singlets tend to be the electronic ground state in bridged systems when they are able to exploit their variational flexibility. This can be prevented, for 
instance, and triplets can then become ground states, when the energetic cost of bending the bridge does not compensate configuration mixing. In this situation, the one-particle density of the singlet is forced to resemble considerably that of the triplet, much like in the case of the Coulson-Fischer HL function in $\mathrm{H}_{2}$. Being now the one-particle energy terms alike in both states, the larger mean interelectron separation of the triplet makes itself felt, and its smaller electron repulsion can make it fall below the singlet. We also find that the local energy of the two magnetic atoms evolves as the total energy of the system, supporting the non-essential energetic role of the bridge in determining the ground spin state. The systems are found to behave as rather localized diradicals, with relatively small local spins on the bridge. Although not essential, exchange pathways can also be sensed with our real space methodology, and we clearly show that they are more important in singlets, as expected.

We try to present our arguments as didactically as possible. First we provide a succinct summary of the model systems and model wavefunctions that we use, together with the real space techniques chosen. Then we show that strict localization leads to a necessarily unbound state in $\mathrm{H}_{2}$ with no singlet-triplet gap. After this we study the $\mathrm{H}-\mathrm{He}-\mathrm{H}$ system and rationalize the singlet-triplet switching through an orbital-based energetic window that paves the way to the subsequent real space analysis.

\section{Basic models and techniques}

We consider the coupling of two $S=1 / 2$ centers without and with an atomic bridge. The first case will be exemplified by the $\mathrm{H}_{2}$ system, and the second by the $\mathrm{H}-\mathrm{He}-\mathrm{H}$ chain. Since we are considering four electrons at most, full configuration interaction (FCI) calculations provide appropriate solutions capturing the full physics of the problem. Minimal basis sets (STO-6G) as well as extended ones (6-311G(p)) will be used. All trends and qualitative results obtained with the extended bases are reproduced with the minimal sets, allowing an immediate fruitful chemical interpretation. 
To perform real space analyses, a partition of space into chemically meaningful regions is in need. We choose the atomic partition provided by the quantum theory of atoms in molecules (QTAIM), ${ }^{27}$ which provides a physically rooted decomposition. Only reduced density matrices (e.g. one- and two-particle densities) are used, so that the formalism is orbital invariant by construction. We also evaluate (see the SI for a succinct presentation) the probability of finding an exact, integer number of electrons in the different atomic regions, the so-called electron distribution functions (EDF), ${ }^{30,31}$ that provide the weights of real space resonance structures, the expectation values of the squared spin operator within an atomic region as well as that of the spin coupling between two regions, ${ }^{32}$ and perform an exact energy decomposition using the interacting quantum atoms methodology. ${ }^{28,29}$ In the latter, the total energy of a system is partitioned as a sum of local intra-atomic or self-energies, $E_{\text {self }}^{A}$, that take into account how much atoms deform when forming molecules, and pairwise additive interatomic interaction energies, $E_{\text {int }}^{A B}$, so that $E=\sum_{A} E_{\text {self }}^{A}+\sum_{A>B} E_{\text {int }}^{A B}$. The atomic self-energies are physically composed of the kinetic energy of their electrons, their attraction to the nucleus, and their mutual interelectron repulsion.

Let us label the magnetic centers as $a$ and $b$. In the absence of bridge, the traditional non-orthogonal Heitler-London solutions for the singlet and triplet are

$$
\psi_{S, T}=N_{ \pm}\left(\varphi_{a}(1) \varphi_{b}(2) \pm \varphi_{b}(1) \varphi_{a}(2)\right) \times f_{S, T},
$$

where $f_{S, T}=(\alpha(1) \beta(2) \mp \beta(1) \alpha(2)) / \sqrt{2}, \varphi_{a}, \varphi_{b}$ are $1 s$ non-orthogonal functions centered at the $a, b$ sites, and $N_{ \pm}=1 / \sqrt{2\left(1 \pm S_{a b}^{2}\right)}$. The covalent singlet HL ansatz provides a very reasonable description of $\mathrm{H}_{2}$ binding, with $D_{e} \approx 96 \mathrm{kcal} / \mathrm{mol} .{ }^{33}$ Mixing it with ionic structures leads to a covalent weight of about 0.76 and a much better $D_{e}=105 \mathrm{kcal} / \mathrm{mol}$. The triplet is unbound, always above the singlet, and $\Delta_{S T}$ adopts the Kahn-Briat expression when $S_{a b}$ is small enough. With a minimal STO-6G basis, $S_{a b} \approx 0.68$ at the equilibrium distance, so that the overlap tails are all but negligible. The spatial part of the wavefunction is symmet- 

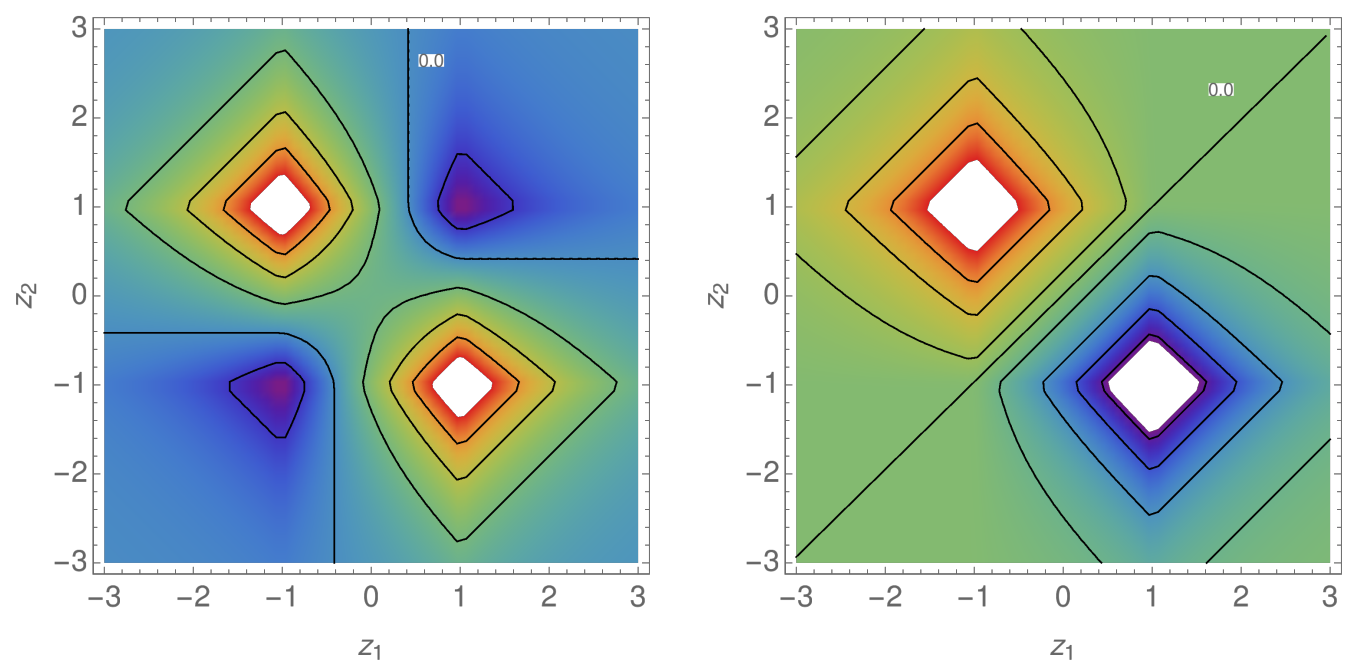

Figure 1: Projection density plot for $\psi_{S, T}^{o}\left(\boldsymbol{r}_{1}, \boldsymbol{r}_{2}\right)$ onto a plane contaning the nuclei in the $\mathrm{H}_{2}$ (left, right) molecule at an internuclear distance $R=2 \mathrm{au}$, as computed with $\varphi=\pi^{-1 / 2} e^{-r}$ 1s functions. The color code goes from dark blue to red, spanning the $[-0.15,+0.15]$ au range. The zero isoline has been highlighted, and white is used at saturation to avoid the nuclear cusps. The two electrons are constrained to move onto the internuclear $z$ axis with nuclei at $(0.0,0.0, \pm 1.0)$ au, the independent axes being the $z_{1}$ and $z_{2}$ coordinates.

ric/antisymmetric for the singlet/triplet, so that the singlet is nodeless, but the triplet is not. This increases the mean interelectron separation in the latter and leads to an electron density that, to first order in $S_{a b}$ equals $\rho(\boldsymbol{r})_{S, T}=\left|\varphi_{a}(\boldsymbol{r})\right|^{2}+\left|\varphi_{b}(\boldsymbol{r})\right|^{2} \pm 2 \varphi_{a}(\boldsymbol{r}) \varphi_{b}(\boldsymbol{r}) S_{a b}$.

Since modern electronic structure theory is MO based, the equivalent orthogonal picture starts with symmetry adapted functions $\phi_{g, u}=1 / \sqrt{2\left(1 \pm S_{a b}\right)}\left(\varphi_{a} \pm \varphi_{b}\right)$. In this variational space there are two ${ }^{1} \Sigma_{g}^{+}$singlet determinants, $\left|\phi_{g} \bar{\phi}_{g}\right|$ and $\left|\phi_{u} \bar{\phi}_{u}\right|$, and one ${ }^{3} \Sigma_{u}^{+}$triplet, whose $M_{S}=0$ component is $1 \sqrt{2}\left(\left|\phi_{g} \bar{\phi}_{u}\right|-\left|\phi_{u} \bar{\phi}_{g}\right|\right)$. The variational space of the singlet is thus larger than that of the triplet. At the mean-field level, $\left|\phi_{g} \bar{\phi}_{g}\right|$ is the singlet ground state, with a $50 \%$ ionic component that can be decreased by CI mixing with $\left|\phi_{u} \bar{\phi}_{u}\right|$, leading to a solution indistinguishable from the covalent plus ionic HL resonance. It is very important to notice that the HL singlet built with $\varphi_{a}$ and $\varphi_{b}\left(\psi_{S}\right.$, Eq. 1) increases its $\left(\varphi_{a}(1) \varphi_{a}(2)+\varphi_{b}(1) \varphi_{b}(2)\right)$ ionic component upon mixing, while the contrary is true when a $\mathrm{CI}(2,2)$ is performed on the Hartree-Fock (HF) determinant, i.e. mixing the $\left|\phi_{g} \bar{\phi}_{g}\right|$ and $\left|\phi_{u} \bar{\phi}_{u}\right|$ determinants. A full 
consideration of these states in real space has already been presented. ${ }^{26}$

Localized (Coulson-Fischer) orthogonal orbitals can be immediately built from the symmetry adapted gerade and ungerade functions: $\phi_{a, b}=1 / \sqrt{2}\left(\phi_{g} \pm \phi_{u}\right)$ (notice the use of $\phi$ instead of $\varphi$ when using orthogonal functions). With these orbitals one can build HL-like singlet and triplet functions,

$$
\psi_{S, T}^{o}=\frac{\left(\phi_{a}(1) \phi_{b}(2) \pm \phi_{b}(1) \phi_{a}(2)\right)}{\sqrt{2}} \times f_{S, T}=\frac{\left(\left|\phi_{a} \bar{\phi}_{b}\right| \pm\left|\phi_{b} \bar{\phi}_{a}\right|\right)}{\sqrt{2}} .
$$

When restated in terms of the canonical orbitals, these are simply the $1 / \sqrt{2}\left(\left|\phi_{g} \bar{\phi}_{g}\right|-\left|\phi_{u} \bar{\phi}_{u}\right|\right)$ function for the singlet and the $1 / \sqrt{2}\left(\left|\phi_{g} \bar{\phi}_{u}\right|-\left|\phi_{u} \bar{\phi}_{g}\right|\right)$ one for the triplet. Obviously, the triplet's space is one-dimensional and remains fixed in the canonical or localized pictures. The orthogonal HL singlet does not. Straightforward application of the Slater-Condon rules leads to $\Delta_{S, T}^{o}=2 K_{a b}$, where the exchange integral is computed over the localized orthogonal $\phi$ 's. The singlet is thus unbound and above the triplet, which is said to fall below the former as a consequence of Hund's rules in the case of two degenerate $\phi_{a, b}$ functions. Noticing that the two determinants differ in two spin-orbitals, the one-particle density matrices $\rho_{1}\left(\boldsymbol{r}_{1} ; \boldsymbol{r}_{1}^{\prime}\right)$ of the two states $\psi_{S, T}^{o}$ are equal, and so are the natural orbitals (with occupation equal to one in the singlet and the triplet), the electron densities, $\rho$, and the kinetic and electron-nucleus energies. Only the pair densities are different, with a $\Delta \rho_{2}\left(\boldsymbol{r}_{1}, \boldsymbol{r}_{2}\right)_{S, T}=2 \phi_{a}\left(\boldsymbol{r}_{1}\right) \phi_{b}\left(\boldsymbol{r}_{1}\right) \phi_{a}\left(\boldsymbol{r}_{2}\right) \phi_{b}\left(\boldsymbol{r}_{2}\right)$ value that provides the $2 K_{a b}$ energy difference. This is a crystal clear example of how a one-particle description (and thus the orbital model) is insufficient to understand even the simplest problems. Interestingly, given the orthogonality of $\phi_{a}$ and $\phi_{b}$, the two orbitals are also orthogonal in any of the two equivalent atomic regions (for instance, in those provided by the QTAIM), so that $\int_{a} \phi_{a}(\boldsymbol{r}) \phi_{b}(\boldsymbol{r}) d \boldsymbol{r}=\int_{b} \phi_{a}(\boldsymbol{r}) \phi_{b}(\boldsymbol{r}) d \boldsymbol{r}=0$. This implies (see the SI) that the localization and delocalization indices, that measure the degree of spatial electron delocalization, ${ }^{34,35}$ are identical in both states, i.e. that the $\psi_{S}^{o}$ singlet is as (de)localized as the triplet $\psi_{T}^{o}$, and that the probability of finding two electrons (see also the SI) in any of 
the atomic regions and none in the other, $p(2,0)$, an orbital invariant descriptor of the ionic character of the wavefunction, is also equal in both states. ${ }^{26}$ For instance, $p(2,0)=0.058$ when a $6-311 \mathrm{G}(\mathrm{p})$ basis set is used at $R=1.42$ bohr. Let us recall that the HF singlet has $p(2,0)=0.25$ (and so the ionic terms weigh exactly 50\%) and that the FCI one decreases this value to $p(2,0) \approx 0.21$. This provides a clear clue about the weirdness of the $\psi_{S}^{o}$ singlet. It displays the same density and the same number of electron pairs in each atomic basin, differing only in their average separation, which is higher in the triplet as a result of the antisymmetry of the same spin pair density, $\rho_{2}^{\sigma \sigma}\left(\boldsymbol{r}_{1}, \boldsymbol{r}_{1}\right)=0$. As it is clear from Fig. 1 , the electrons try to stay away from each other in both states, but the two negative peaks along the main diagonal in the singlet give rise to a larger electron-electron repulsion. It is also interesting to understand how $p(2,0)$, whose $z$ projection is obtained after integrating the density displayed in Fig. 1 in the $\left(z_{1} \geq 0, z_{2} \geq 0\right)$ or the $\left(z_{1} \leq 0, z_{2} \leq 0\right)$ quadrants, can be equal in both states. The singlet displays a wider nodal line than the triplet, violating both the nodeless nature of the ground state, and the Lieb-Mattis theorem ${ }^{36}$ for two electron systems. We stress that it is this artificially localized state which is used in one way or another to build magnetic coupling intuition in the orbital model. Both the bare $K_{a b}$ values and the weights of the ionic mixing that transforms the $\psi_{S}^{o}$ singlet into the ground state are as pathological as the $\psi_{S}^{o}$ function itself. By preventing electron delocalization through the use of OLOs we artificially force the triplet below the singlet. And, as we show in the next section, not even in this case we avoid ionic mixing.

\section{Electron delocalization and magnetic coupling}

We now show that fully localized electrons prevent binding and any singlet-triplet splitting in our $\mathrm{H}_{2}$ model. The proof is simple. Let us consider that each of the two electrons is spatially localized in one of the two equivalent atomic domains, and let us use the real space energetic stance provided by IQA (see the SI). The total energy of the system is 
$E=E_{\text {self }}^{a}+E_{\text {self }}^{b}+E_{\text {int }}^{a b}$. Since the electrons are strictly localized, $E_{\text {self }}$ can be built from the one-particle density matrix only, for no intra-domain electron-electron repulsion exists. It is thus the $\operatorname{trace}^{28}$ of the $\mathrm{H}$ atom one-particle Hamiltonian over a density which is not variational, being thus above its in vacuo energy. Similarly, since one electron sits always in $a$ while the other sits in $b$, the pair density is just the product of the densities for each electron, so that $E_{i n t}^{a b}$ is the classical electrostatic interaction of two point nuclear charges and two symmetric extended electron densities. By Earnshaw's theorem, ${ }^{37}$ this interaction energy is positive, so the final binding energy of the system is destabilizing at any internuclear distance: localization prevents binding.

We can now build an explicit solution for singlet and triplet states by imagining general $\phi_{a}$ and $\phi_{b}$ OLOs which are different from zero only in the $a$ or $b$ domains, respectively, i.e. $\phi_{a}(\boldsymbol{r})=0$ if $\boldsymbol{r} \in b$, and viceversa. The complex squares of these orbitals provide the electron density in each region. Properly symmetrized state functions for the singlet and triplet are immediately built as in Eq. 2. From them we can easily find that the one-particle density matrix is $\rho_{1}\left(\boldsymbol{r} ; \boldsymbol{r}^{\prime}\right)=\phi_{a}(\boldsymbol{r}) \phi_{a}^{*}\left(\boldsymbol{r}^{\prime}\right)+\phi_{b}(\boldsymbol{r}) \phi_{b}^{*}\left(\boldsymbol{r}^{\prime}\right)$ and that the pair density becomes $\rho_{2}\left(\boldsymbol{r}_{1}, \boldsymbol{r}_{2}\right)=\left|\phi_{a}\left(\boldsymbol{r}_{1}\right)\right|^{2}\left|\phi_{b}\left(\boldsymbol{r}_{2}\right)\right|^{2}+\left|\phi_{b}\left(\boldsymbol{r}_{1}\right)\right|^{2}\left|\phi_{a}\left(\boldsymbol{r}_{1}\right)\right|^{2}$, regardless of whether the state is a singlet or a triplet, since $\phi_{a}\left(\boldsymbol{r}_{1}\right) \phi_{b}\left(\boldsymbol{r}_{1}\right) \phi_{a}\left(\boldsymbol{r}_{2}\right) \phi_{b}\left(\boldsymbol{r}_{2}\right)$ is permanently zero for any $\boldsymbol{r}_{1}$ and $\boldsymbol{r}_{2}$ values. Since no difference exists now in the pair densities, $\Delta E_{S, T}=0$. Thus, a strict localization in real space prevents magnetic coupling.

In our opinion, this academic example shows the potential problems of any orbital-based decomposition of $\Delta E_{S, T}$. The conducting wire filling the gaps is the amount of electron delocalization. If absent, no ionic structures are present, since the probability that the two electrons be found in the same region vanishes, $p(2,0)=p(0,2)=0$, so $p(1,1)=1$, and no spin-triplet splitting arises. If present, both $p(2,0)$ and $p(0,2)$ are non-zero, and any OLOs-based description will also lead to positive $K_{a b}$ and $t_{a b}^{2} / U$ integrals which will conspire to offer the final orbital invariant $J$ value. An eye-catching summary of these ideas can be found in Fig. 2. Separating artificially ferro and antiferromagnetic components depends on 

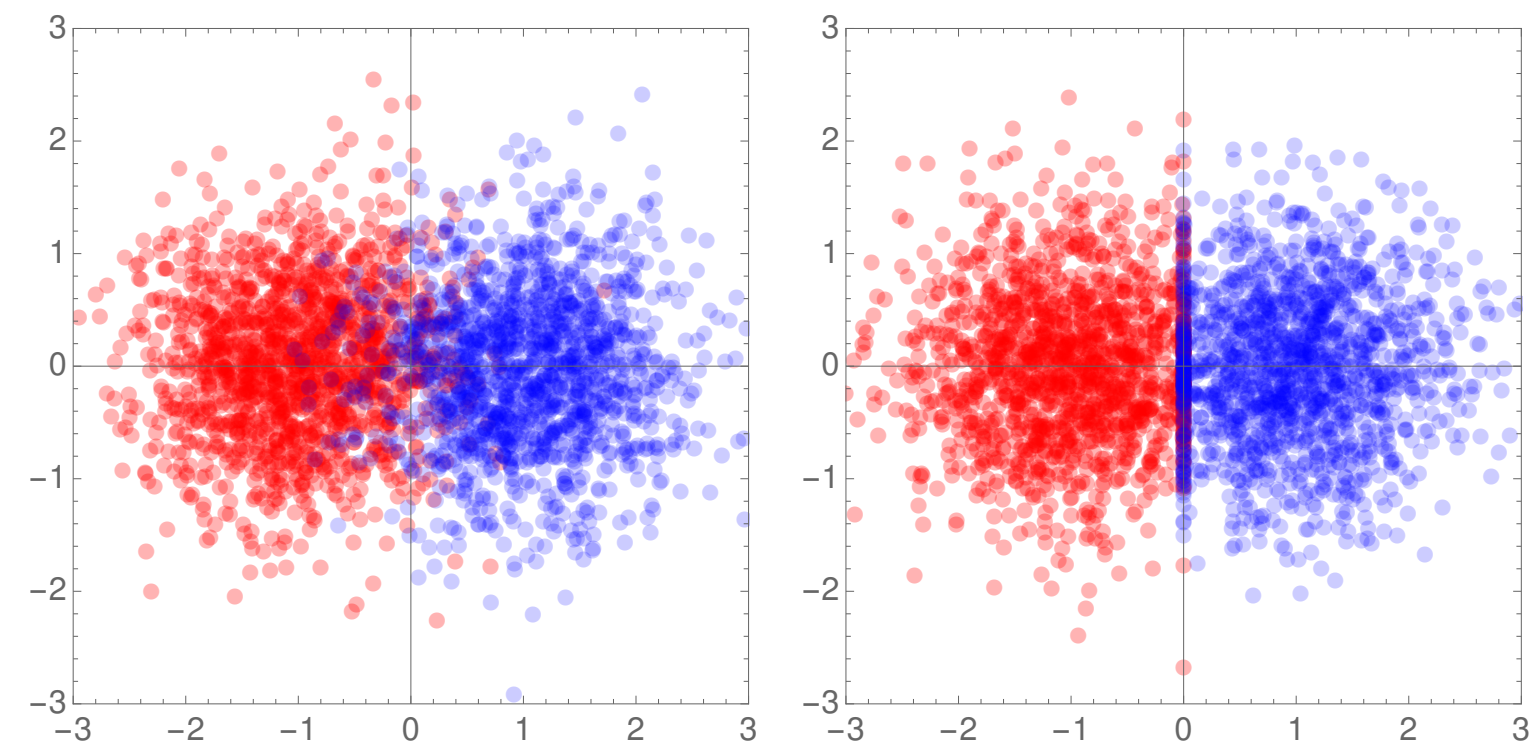

Figure 2: Statistical sampling of the positions of the two electrons of a model $\mathrm{H}_{2}$ molecule with an internuclear distance of $R=2$ bohr. In the left and right panels we allow and forbid, respectively, electron delocalization between the two equivalent atomic regions. The distribution has been projected onto a plane that contains the internuclear axis, with the nuclei at $0.0,0.0, \pm 1.0 \mathrm{bohr}$, so that the atomic division becomes the $y$ axis. The two electrons are artificially labeled by color. Notice that when electrons delocalize, the probability that the two of them lie in, e.g., the left atom, as given by the $p(2,0)$ ionic probability described in the text, is non zero. In this case, binding as well as singlet-triplet splitting, occurs. On the contrary, when delocalization is forbidden, the blue (red) electron remains always on the right (left) regions. Any state like this is unbound and its singlet-triplet gap vanishes. 
choosing a given orbital picture. As we have shown, allowing electrons to delocalize switches on both $K_{a b}$ and ionic mixing, simultaneously. Considering them independent actors in the coupling game is not justified.

\section{Coupling through an atomic bridge: the $\mathrm{H}-\mathrm{He}-\mathrm{H}$ orbital}

\section{image}

We now turn to consider the possibly simplest model system that displays a structural dependent switch of magnetic states: the symmetric H-He-H aggregate. Aside from van der Waals stabilized complexes, the ground state potential energy surface of this system collapses over a free He atom and a $\mathrm{H}_{2}$ molecule, so we simply use constrained geometries to show our arguments. It is easily found that the system's ground state is a singlet for linear configurations and a triplet when the $\mathrm{H}-\mathrm{He}-\mathrm{H} \alpha$ angle is close to $90^{\circ}$. This order is maintained for a wide range of $\mathrm{H}-\mathrm{He} R$ distances.

Being a four electron system, the $\mathrm{H}-\mathrm{He}-\mathrm{H}$ aggregate can be solved at the FCI level effortlessly with large basis sets. Although the quantitative energies and $J$ splittings do obviously change with the quality of the basis set, interestingly the singlet-triplet state ordering is maintained even with minimal descriptions, so that clear qualitative chemical pictures can be drawn easily. Notice that in a minimal description no functions with $l>0$ are used in the cases examined, so that both polarization effects as well as participation of higher energy virtual pathways have to be abandoned as key actors in the coupling. We have performed STO-6G (minimal) as well as 6-311G(p) (extended) FCI calculations at linear (L) and bent (B) geometries that exemplify the singlet-triplet switching. To understand the role of the atomic bridge, we have also added calculations for the isolated $\mathrm{H}_{2}$ molecule at the same H-H separations. Since $\Delta E_{S, T}$ falls quickly with $R$, we report data for $R$ values which provide splittings in the usual magnetic energy scale. We enforce $D_{2 h}$ and $C_{2 v}$ geometries for the $\mathrm{L}$ and $\mathrm{B}$ geometries, but it will suffice us to label states as $a_{1}$ or $b_{1}$ in $C_{2 v}$ symmetry. Our 
main discussion will use $R_{\mathrm{HeH}}=1.2587 \AA$, and $\alpha$ either $180^{\circ}$ or $91.3^{\circ}$, with $R_{\mathrm{HH}}=1.8000 \AA$ in the latter case. When analyzing triplets, we compute always the $M_{S}=0$ component. To better understand the arguments below, Fig. 3 shows a sketch of the STO-6G orbital picture for both the singlet and the triplet states.
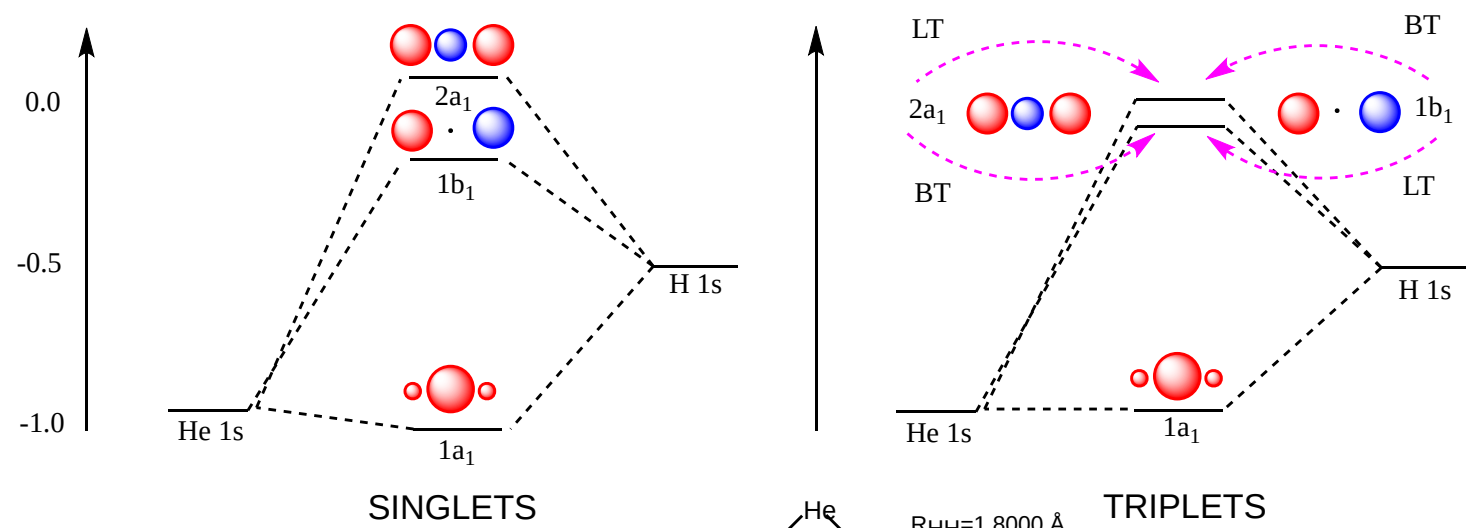

$\mathrm{RHH}=2.5174 \AA \quad \mathrm{H}-\mathrm{He}-\mathrm{H}$

Figure 3: STO-6G orbital diagram for the minimum energy singlet and triplet states of $\mathrm{H}-\mathrm{He}-\mathrm{H}$ in both the linear and bent geometries. The canonical orbital energies $\epsilon$ have been drawn approximately on an energy scale in au. The magnetic orbitals of the two singlets and the two triplets show similar $\epsilon$ 's. Notice the ordering change in the $1 b_{1}$ and $2 a_{1}$ functions in the linear and bent triplet.

The extended calculations in $\mathrm{H}_{2}$ yield academic results. At $R_{\mathrm{HH}}=2.5174 \AA, \Delta E_{S, T}=$ $-6.80 \mathrm{mE}_{h}$, and the wavefunction is dominated by two configurations, $\psi_{S} \approx 0.795\left|a_{1} \bar{a}_{1}\right|-$ $0.571\left|b_{1} \bar{b}_{1}\right|+\ldots$, that lead to $a_{1}$ and $b_{1}$ natural orbitals with occupations equal to 1.302 and 0.698 , respectively. At $R_{\mathrm{HH}}=1.8000 \AA, \Delta E_{S, T}=-49.0 \mathrm{mE}_{h}$, and the wavefunction increases its single-determinant character, $\psi_{S} \approx 0.912\left|a_{1} \bar{a}_{1}\right|-0.363\left|b_{1} \bar{b}_{1}\right|+\ldots$, with natural orbital occupancies equal to 1.680 and 0.318 , respectively. The triplets are in both cases almost pure, with $\psi_{T} \approx 0.707\left|a_{1} \bar{b}_{1}\right|-0.707\left|b_{1} \bar{a}_{1}\right|$, as it is well known. Let us recall that both geometries are well past equilibrium, so that in the singlets the kinetic energy is lower than at dissociation, while the contrary is true for the triplets, which show smaller interelectron repulsion than that of their corresponding singlets thanks to their spatially antisymmetric wavefunctions. The singlets are in both cases rather far from the $\psi_{S}^{o}$ limit already discussed. 
It is an interesting exercise to construct a pure $\psi_{S}^{o}$ function from the triplet's canonical orbitals (as done in conventional MO theory). At $R_{\mathrm{HH}}=2.5174 \AA$, for instance, this HL Coulson-Fischer singlet lies $2.00 \mathrm{mE}_{h}$ above the FCI triplet, a quantity which is essentially equal to the $2.07 \mathrm{mE}_{h}$ increase in interelectron repulsion suffered by the triplet on going to the artificial Coulson-Fischer state.

Introduction of the He atomic bridge yields singlet linear and triplet bent ground states, and now $\Delta E_{S, T}=-21.2$ and $+4.2 \mathrm{mE}_{h}$ for the linear and bent configurations, respectively. The preferentially occupied canonical orbitals are a $1 a_{1}$ function which is basically the $1 \mathrm{~s} \mathrm{He}$ orbital and two $2 a_{1}$ and $1 b_{1}$ functions in which the symmetric and antisymmetric combination of the $1 \mathrm{~s} \mathrm{H}$ orbitals dominate. We will call the latter the active $a_{1}$ and $b_{1}$ orbitals. The occupancy of the $1 a_{1}$ natural orbital is greater than 1.98 in all cases, so that the orbital game basically involves the $a_{1}$ and $b_{1}$ functions, as expected.

Table 1: 6-311G(p) FCI data for the linear (L) and bent (B) H-He-H geometries described in the text. S stands for singlet and $\mathrm{T}$ for triplet, and $\phi$ is the same $a_{1}$ or $b_{1}$ function as that written explicitly in the determinant for the singlets, while it is the other one in the triplets. $n$ are the occupation numbers of the $2 a_{1}$ and $1 b_{1}$ active natural orbitals. $\mathrm{K}$, ne, ee are the total kinetic, nucleus-electron, and electron repulsion energies, and $\epsilon$ the canonical orbital energies. All data in au.

\begin{tabular}{c|rr|rr}
\hline & LS & LT & BS & BT \\
\hline $\mathrm{E}$ & -3.8328 & -3.8116 & -3.8102 & -3.8144 \\
$\mathrm{~K}$ & 3.9700 & 4.0902 & 4.1079 & 4.0788 \\
$\mathrm{ne}$ & -12.3468 & -12.4305 & -12.6211 & -12.5804 \\
$\mathrm{e}$ & 2.6523 & 2.6369 & 2.7274 & 2.7116 \\
$c\left(\left|a_{1} \bar{\phi}\right|\right)$ & -0.4983 & 0.7033 & 0.7360 & 0.7040 \\
$c\left(\left|b_{1} \bar{\phi}\right|\right)$ & 0.8601 & -0.7033 & -0.6288 & -0.7040 \\
$n\left(a_{1}\right)$ & 0.5044 & 0.9993 & 1.1700 & 0.9999 \\
$n\left(b_{1}\right)$ & 1.4942 & 0.9992 & 0.8282 & 0.9997 \\
$\epsilon\left(a_{1}\right)$ & -0.0269 & -0.0994 & -0.0639 & -0.1585 \\
$\epsilon\left(b_{1}\right)$ & -0.3124 & -0.1854 & -0.2854 & -0.1286 \\
\hline
\end{tabular}

Table 1 summarizes the extended FCI data. All wavefunctions are dominated by two configurations, and the triplets are very close to the open shell HF solution (or to the HL 
function) due to their limited variational space. It is of utmost importance that the linear singlet (LS) preferentially occupied determinant is $\left|b_{1} \bar{b}_{1}\right|$, while the $\left|a_{1} \bar{a}_{1}\right|$ one dominates the bent one $(\mathrm{BS})$, as in dihydrogen. Considering a mean-field (restricted open shell for the $M_{S}=1$ triplet) solution helps in the discussion. The single-determinant LS, with configuration $1 a_{1}^{2} 1 b_{1}^{2}$ and $E_{S}=-3.6749 \mathrm{au}$, is way above the LT, with configuration $1 a_{1}^{2} 2 a_{1} 1 b_{1}$ and $E_{T}=-3.7746$ au. Moreover, a $1 a_{1}^{2} 2 a_{1}^{2}$ singlet built with the triplet's orbitals lies at $E=-3.4788 \mathrm{au}$. The two nodal planes of the $\phi_{a_{1}}$ function give rise to a considerably higher kinetic energy, so that electrons prefer occupying the one-node $\phi_{b_{1}}$ function if forced on a single determinant. This gives rise to the linear triplet, with only one $a_{1}$ electron, falling below the singlet. The bridging atom thus acts as a Pauli barrier to the pumping of electrons into the $\mathrm{H}-\mathrm{H}$ internuclear region, that now contains the $\mathrm{He}$ atom. In its absence, the $\mathrm{H}_{2}$ subsystem prefers occupying the $\phi_{a_{1}}$ function. In its presence, this becomes a double node orbital, which is avoided. As shown in Table 1, configuration mixing renders a linear singlet ground state thanks to a sharp decrease in the kinetic energy.

Restriction to a minimal STO-6G basis, where no orbital polarization is allowed and the wavefunction structure is severely constrained, changes the quantitative, but not the qualitative picture. As expected, energy differences are decreased by the much lower orbital flexibility, but $\Delta E_{S, T}=-15.1$ and $+1.8 \mathrm{mE}_{h}$ in the $\mathrm{L}$ and $\mathrm{B}$ geometries.

Understanding the switch to a triplet ground state in the bent configuration starts by noticing that it clearly fulfills the expectations from the HTH model. Using the triplet's orbitals, the HTH $\left(\epsilon_{a_{1}}-\epsilon_{b_{1}}\right)^{2}$ expression introduces a much larger antiferromagnetic term in the linear than in the bent configuration. Notice that no Kahn-Briat orthogonality arguments can be used here. From the physical perspective, it is interesting to acknowledge how close the energies of the BS, LT, and BT states are. This is particularly striking, since the bent $\mathrm{H}-\mathrm{H}$ distance is considerably shorter than the linear one. Physically, it means that approaching the $\mathrm{H}$ atoms in the triplets does not vary the energy substantially, so that almost perfect nuclear shielding occurs that avoids the increased nuclear repulsions. Since symmetry constrains the 
triplet functions (just notice the very close to 1.0 occupation numbers of the $a_{1}, b_{1}$ natural orbitals or their very close to $\pm 1 / \sqrt{2}$ CI coefficients), an orbital localization leads to almost perfectly localized $\mathrm{H}$ functions. Contrarily, the LS is much more delocalized and, upon bending, it has to respond in order to shift electron density into the $\mathrm{H}-\mathrm{H}$ region to shield the enlarged proton-proton repulsion. Since the $b_{1}$ function has a nodal plane forbidding this density accumulation, the response implies increasing the $\phi_{a_{1}}$ contribution, so that the coefficients of the $\left|a_{1} \bar{a}_{1}\right|$ and $\left|b_{1} \bar{b}_{1}\right|$ determinants approach those in the $\psi_{S}^{o}$ model. With this, its kinetic energy and electron repulsion increase substantially upon bending (Table 1), but the ne attraction does its work to counteract the +0.084 au increase in the nn repulsion. In this process, the LS wavefunction approaches the $\psi_{S}^{o}$ model, and the lower electron repulsion of the triplet does the trick.

Summarizing, the atomic bridge's Pauli barrier drives the two electrons of the $\mathrm{H}_{2}$ subsystem to prefer a $b_{1}^{2}$ configuration over a $a_{1}^{2}$ one in the LS. Configuration mixing leads to an inversion of the magnitude of the CI coefficients with respect to the free $\mathrm{H}_{2}$ molecule, with an occupation number of the $b_{1}$ natural orbital larger than one and close to 1.5 (the $b_{1}$ occupation in the bare dihydrogen at the same internuclear separation is 0.698). On bending, the need to pump charge in between the hydrogens changes the bonding mode to $a_{1}^{2}$, giving rise to a considerable electron reorganization that approaches the singlet to the weird CoulsonFischer model. All triplets, on the contrary, are well localized at any geometry, suffering much smaller energy changes. It is thus possible to use orbital arguments to understand the singlet-triplet switching without invoking the HTH paradigm. Now we turn to show that a real space analysis provides a directly consistent narrative of the above ideas. 


\section{Coupling through an atomic bridge: the alternative real}

\section{space picture}

The analysis of wavefunctions in real space provides strong orbital invariant physical images. Local energies, electron localization and delocalization descriptors, the probabilities of different electron distributions, local spins, etc, are all available once an initial partition of the space into chemically appealing regions is available. As noted, we choose the QTAIM partition. A summary of the real space artillery can be found in the SI.

Table 2: 6-311G(p) FCI real space data for the $\mathrm{H}_{2}$ molecule at the linear (L) and bent (B) $\mathrm{H}-\mathrm{He}-\mathrm{H}$ geometries. $\mathrm{S}$ stands for singlet and $\mathrm{T}$ for triplet. All data in au. The self-energy of the $\mathrm{H}$ atom in vacuo is -0.49981 au.

\begin{tabular}{c|rr|rr}
\hline & $\mathrm{LS}$ & $\mathrm{LT}$ & $\mathrm{BS}$ & $\mathrm{BT}$ \\
\hline$\rho_{b c p}$ & 0.0065 & 0.0049 & 0.0289 & 0.0116 \\
$\nabla^{2} \rho_{b c p}$ & 0.0103 & 0.0137 & 0.0176 & 0.0453 \\
$E_{\text {self }}^{\mathrm{H}}$ & -0.4956 & -0.4954 & -0.4866 & -0.4826 \\
$E_{\text {int }}$ & -0.0128 & -0.0063 & -0.0585 & -0.0176 \\
$\delta^{\mathrm{HH}}$ & 0.0920 & 0.0427 & 0.3503 & 0.1042 \\
$p(2,0)$ & 0.0230 & 0.0107 & 0.0876 & 0.0347 \\
$S_{\mathrm{H}}^{2}$ & 0.7155 & 0.7340 & 0.6186 & 0.6979 \\
\hline
\end{tabular}

We start again by summarizing the bare $\mathrm{H}_{2}$ results, which can be found in Table 2 . At both geometries we are still in the closed-shell regime, where the Laplacian at the internuclear midpoint (the QTAIM bond critical point, bcp) is positive. As expected and known, the larger $\phi_{a 1}$ occupation number of the singlet yields larger bonding densities, and less positive Laplacians. Also to be noticed are the larger deformation energies (defined as the selfenergy cost of passing from an in vacuo atom to the atom-in-the-molecule situation, $E_{\text {def }}^{A}=$ $\left.E_{\text {self }}-E_{\text {self }}^{A, 0}\right)$ of the $\mathrm{H}$ atoms in the triplet as $R_{\mathrm{HH}}$ decreases, as well as the considerably larger electron delocalization indices found in the singlet. Yet, deformations are small in the regime examined, peaking at about $11 \mathrm{kcal} / \mathrm{mol}$ in the BT case. The amount of ionic structures, given by $p(2,0)=p(0,2)$, shows that even the triplet state leads to a measurable 
delocalization and non-vanishing probability to find the two electrons in the same atomic region: the weight of ionic structures is approximately $5,2,17$, and $7 \%$ for the LS, LT, BS, and BT states, respectively. The local spin analysis ${ }^{32}$ is compatible with the small overall delocalization. All systems are clear diradical species, coupled to singlet or triplet total spin states. The diradical character is larger in the triplet, also as expected.

Table 3: 6-311G(p) FCI real space data for the H-He-H system at the linear (L) and bent (B) geometries. S stands for singlet and $\mathrm{T}$ for triplet. The isolated FCI energies of the $\mathrm{H}$ and He atoms are -0.49981 and -2.89057 au, respectively. All data in au.

\begin{tabular}{c|rr|rr}
\hline & LS & LT & BS & BT \\
\hline$\rho_{b c p}$ & 0.0781 & 0.0685 & 0.0676 & 0.0705 \\
$\nabla^{2} \rho_{b c p}$ & 0.0917 & 0.1592 & 0.1750 & 0.1556 \\
$\rho_{\text {bcp* }}$ & - & - & 0.0268 & 0.0260 \\
$\nabla^{2} \rho_{b c p *}$ & - & - & 0.1323 & 0.1373 \\
$Q^{\mathrm{He}}$ & -0.0123 & -0.0218 & -0.0380 & -0.0249 \\
\hline$E_{\text {self }}^{\mathrm{He}}$ & -2.7712 & -2.7832 & -2.7872 & -2.7813 \\
$E_{\text {self }}^{\mathrm{H}}$ & -0.4685 & -0.4659 & -0.4603 & -0.4626 \\
$E_{\text {self }}^{\mathrm{HH}}$ & -0.9423 & -0.9306 & -0.9839 & -0.9881 \\
$E_{\text {int }}^{\mathrm{HeH}}$ & -0.0597 & -0.0489 & -0.0468 & -0.0504 \\
$E_{\text {int }}^{\mathrm{HH}}$ & -0.0052 & 0.0012 & -0.0082 & -0.0062 \\
\hline$\delta^{\mathrm{HeH}}$ & 0.2442 & 0.2096 & 0.1967 & 0.2113 \\
$\delta^{\mathrm{HH}}$ & 0.0824 & 0.0138 & 0.0750 & 0.0619 \\
$\delta^{3}$ & 0.0107 & 0.0061 & 0.0130 & 0.0146 \\
\hline$p(1,2,1)$ & 0.7278 & 0.7919 & 0.7792 & 0.7714 \\
$p(0,3,1)$ & 0.0571 & 0.0529 & 0.0537 & 0.0537 \\
$p(2,1,1)$ & 0.0528 & 0.0439 & 0.0364 & 0.0432 \\
$p(2,2,0)$ & 0.0228 & 0.0051 & 0.0192 & 0.0161 \\
\hline$S_{\mathrm{He}}^{2}$ & 0.198 & 0.181 & 0.154 & 0.183 \\
$S_{\mathrm{H}}^{2}$ & 0.663 & 0.712 & 0.690 & 0.717 \\
$\boldsymbol{S}_{\mathrm{He}} \cdot \boldsymbol{S}_{\mathrm{H}}$ & -0.050 & -0.030 & -0.039 & -0.017 \\
$\boldsymbol{S}_{\mathrm{H}} \cdot \boldsymbol{S}_{\mathrm{H}}$ & -0.564 & +0.209 & -0.613 & +0.200 \\
\hline & & & &
\end{tabular}

Examining the inclusion of the He bridge in real space allows us to follow the change in all these properties smoothly. A summary of the data can be found in Table 3. Taking a look at just plain density descriptors, it is clearly found that all interactions are closed-shell like with positive Laplacians, and that the He-H bcps display density values which correlate with the observed ground state. This has been found to be general, so that the interaction energy 


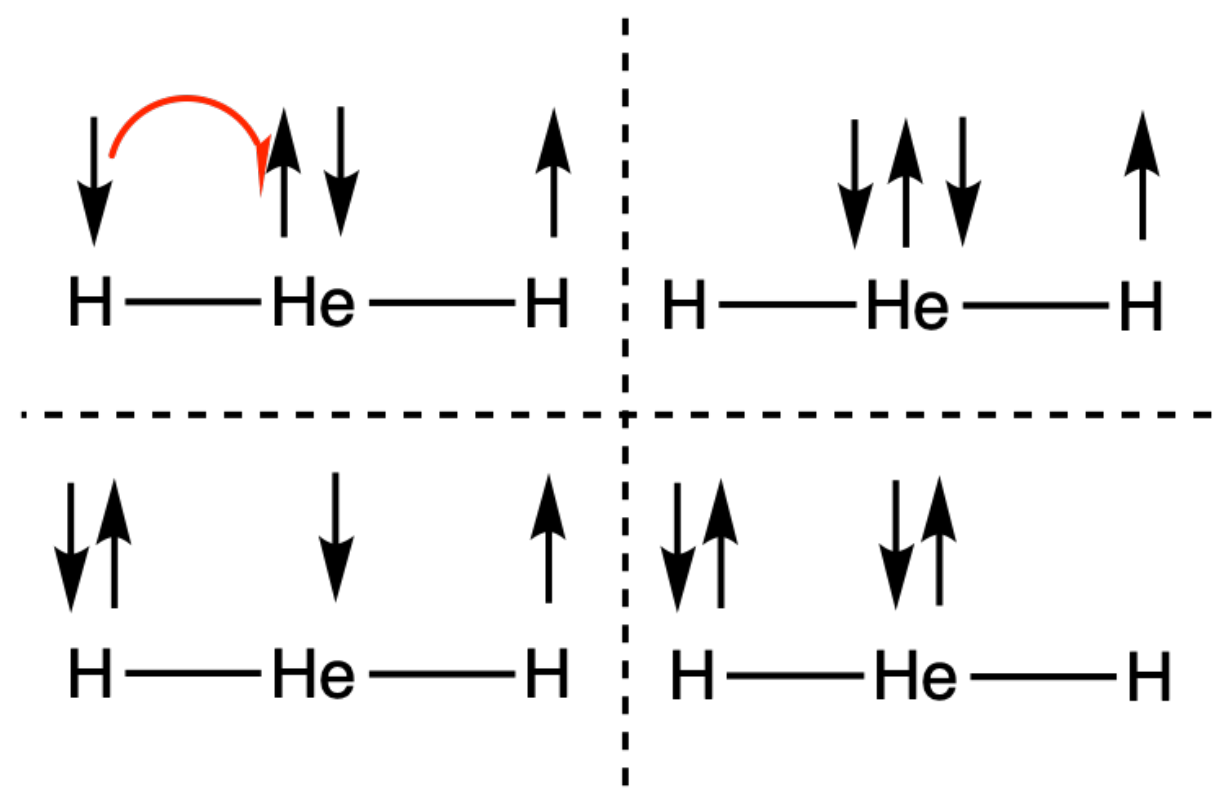

Figure 4: FCI/6311-G(p) dominant electron distributions of the several states of H-He-H, starting with the $(1,2,1)$ one on the top-left panel. Both the top-right and bottom-left arrangements are $\mathrm{H}$-He one-electron exchanges, but the bottom-right one, $(2,2,0)$, implies either one direct $\mathrm{H}-\mathrm{H}$ or two coupled $\mathrm{H}-\mathrm{He}$ one-electron exchanges.

between the magnetic center and the bridge predicts the ground state. For the bent structures there is no $\mathrm{H}-\mathrm{H}$ bcp, but we have also added density descriptors at the $\mathrm{H}-\mathrm{H}$ midpoint (bcp* in the Table). Notice how the BS pumps density into the bcp* region, as expected from orbital arguments. Topological charges talk about a considerably electronegative He atom. This electronegativity is maximal when the interaction energy between the magnetic centers and the bridge $\left(E_{i n t}^{\mathrm{HeH}}\right)$ is small, as in the BS case, and decreases as the He-H interaction increases.

We turn now to the energetic realm. By comparing with the bare results of Table 2, it is found that the $\mathrm{H}$ atoms deformation is around $20 \mathrm{kcal} / \mathrm{mol}$ larger in the bridged systems. This is accompanied by sizable He deformation energies that reach $90 \mathrm{kcal} / \mathrm{mol}$. The $E_{\text {self }}^{\mathrm{He}}$ values for the LT, BS, and BT systems are similar, in agreement with their overall energies, and the larger He deformation energy in LS points toward a more intense bonding. This is corroborated by comparing the $E_{i n t}^{\mathrm{HeH}}$ energies, which show a larger $\mathrm{HeH}$ interaction in this system. Notice that this descriptor does also correlate with the ground state, reinforcing 
the previous paragraph. Notice also that the behaviour of $E_{\text {self }}^{\mathrm{He}}$ on going from the singlet to the triplet differs in the $\mathrm{L}$ and $\mathrm{B}$ geometries. This again reflects the different $\mathrm{H}$-He bonding strengths.

An important insight is obtained from examining the energy of the $\mathrm{H}_{2}$ subsystem in the triatomic. This is the $E_{\text {self }}^{\mathrm{HH}}$ quantity, which can be directly compared with the bare $\mathrm{H}_{2}$ energy. The linear geometries distort more intensely the $\mathrm{H}_{2}$ subsystem than the bent ones, as expected, but, most importantly, the energetic ordering of the subsystem is again correlated with the total ground state. This means that if the electronic structure of the dihydrogen subsystem is appropriately modified by the bridge but the latter is afterwards ignored, the sign of $J$ is appropriately predicted. This casts doubts on the necessity of the bridge to understand magnetic couplings, see below. Quantitatively speaking, at the linear configuration $\Delta E_{S, T}$ for the $\mathrm{H}_{2}$ subsystem is enhanced from -6.8 to $-11.7 \mathrm{mE}_{h}$ on introducing the bridge, a value to be compared to the total $\mathrm{H}-\mathrm{He}-\mathrm{H} \Delta E_{S, T}$ of $-22.6 \mathrm{mE}_{h}$. This shows that the presence of the He atom is not necessary to yield the correct ground state, but that it clearly contributes to the singlet-triplet gap, as expected from all the accumulated MO knowledge. Similarly, at the bent geometry, we pass from -49.0 to +4.2 to $+2.8 \mathrm{mE}_{h}$, respectively, and the He atom contributes stabilizing slightly the singlet with respect to the triplet as a result of the smaller He deformation in the singlet.

Regarding electron delocalization, electrons prefer to delocalize with the He bridge, as evidenced by the much larger $\delta^{\mathrm{HeH}}$ than $\delta^{\mathrm{HH}}$ values in all cases. The former does also correlate with the preferred ground state, so that increased He-H interaction leads to smaller energy. We stress that the smaller $E_{i n t}^{\mathrm{HeH}}$ in the BS with respect to the BT indicates that the electronic structure reorganization that is needed for the BS to minimize its energy and shield the increased nn repulsions upon bending hinders direct $\mathrm{HeH}$ delocalization, in complete agreement with the previous orbital analysis. The HH delocalization index behavior is also illuminating. In the linear geometries it is much larger in the singlet, which displays a value similar to that found in the bare dihydrogen molecule. Contrarily, the BS and 
BT show similar $\delta^{\mathrm{HH}}$ values, which contrast with the bare $\mathrm{H}_{2}$ data of Table 2 . The threecenter delocalization index, measuring simultaneous three-body population fluctuations, ${ }^{38}$ is positive and small, correlating again with the ground state preference.

Let us jump to consider the role of ionic structures. A reasonable route to identify exchange pathways in real space is the consideration of the probability of finding different electron arrangements, as found in the fourth set of rows in Table 3 and in Fig. 4. We consider the atoms in the $\mathrm{H}, \mathrm{He}, \mathrm{H}$ order, so that $p(1,2,1)$ refers to the probability of finding one electron in each $\mathrm{H}$ atom and two in Helium. The neutral structure with one electron in each $\mathrm{H}$ atom and two in the He bridge dominates, being a minimum in the LS, and a maximum in the LT. As expected from the HTH rationale, the one-electron exchanges from $\mathrm{H}$ to $\mathrm{He}$, as given by $p(0,3,1)$ and $p(2,1,1)$, are maximum in the LS, which corroborates the enhancing role of the bridge in stabilizing the singlet in the linear geometry. The sum of both probabilities correlates with the He-H interaction, and tells the traditional story about how exchange with the bridge's electrons is facilitated in singlets but not in triplets. A relevant point here is that it is $p(2,1,1)$ which is considerably hindered in the BS, not $p(0,3,1)$. This has a very simple rationalization in terms of our basic shielding argument. Too many electrons in the $\mathrm{H}$ regions do not help in this case (they increase the electron repulsion between the two $\mathrm{Hs})$. Finally, $p(2,2,0)$ is a measure of direct $\mathrm{H}-\mathrm{H}$ delocalization. Comparing it with $p(2,0)$ in the bare $\mathrm{H}_{2}$ molecule gives rise to the same narrative already commented when explaining the $\delta^{\mathrm{HH}}$ values. It is maximum and minimum for the LS and LT systems, and the two bent systems provide similar small values.

We leave the local spin analysis ${ }^{32}$ for the end of this section. A pictorial representation is found in Fig. 5. It is first noteworthy to notice that the He atom holds a residual, though nonnegligible squared spin. This is a result of H-He delocalization and, in consequence, largest for LS, and smallest for BS. This spin transfer leads to a partial quenching of the spin at the magnetic centers. It would be interesting to examine to what point this affects the hypothesis leading to Heisenberg modeling in real magnetic systems. Secondly, the local spins of the H 


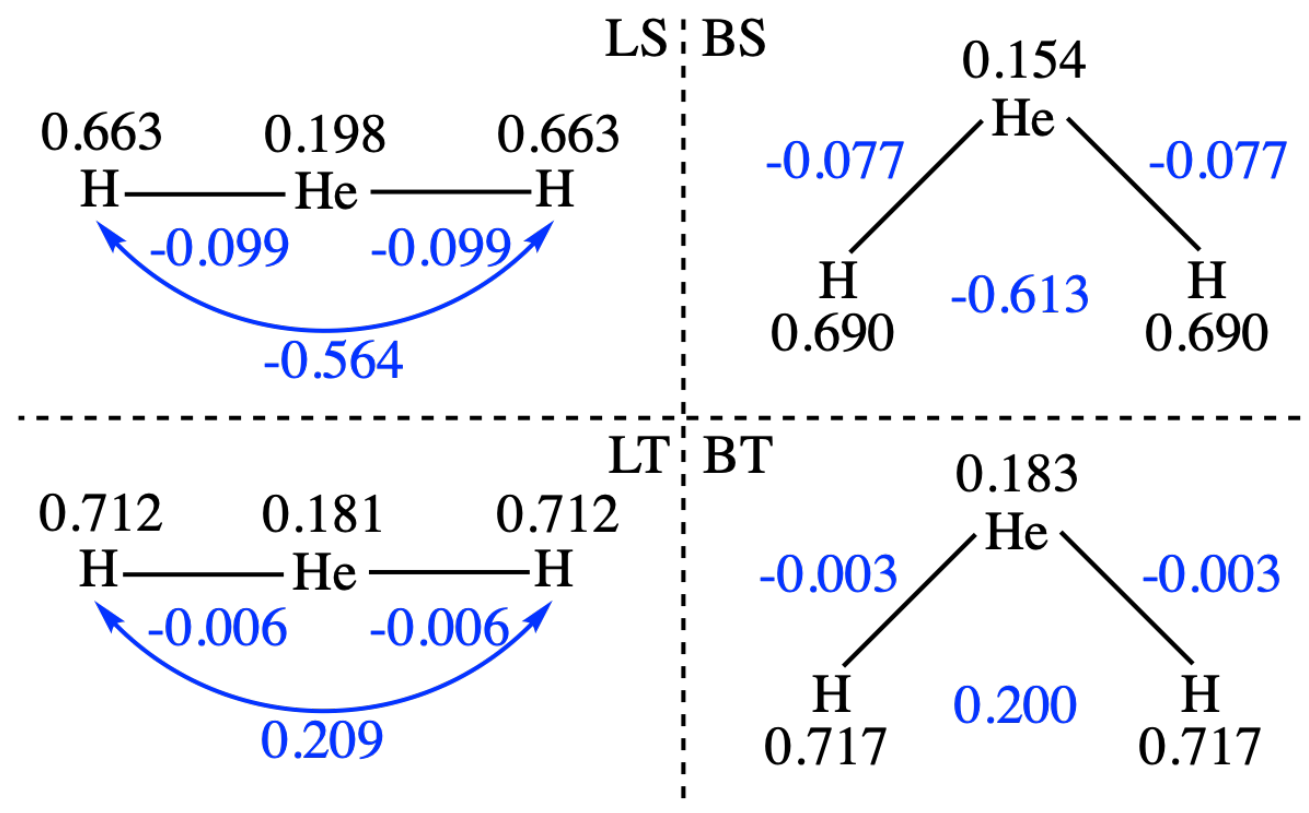

Figure 5: FCI/6311-G(p) local spin analysis for the H-He-H system. $\left\langle S_{A}^{2}\right\rangle$ values in black, $\left\langle\boldsymbol{S}_{A} \cdot \boldsymbol{S}_{B}\right\rangle$ couplings in blue.

atoms are rather large in all cases, approaching the strictly localized values of $3 / 4=0.75$. In this sense, all the systems behave as rather localized diradical species. The worst localized LS system shows the largest deviations of this limiting model. As the atomic spin couplings are regarded, recall that negative/positive $\boldsymbol{S}_{i} \cdot \boldsymbol{S}_{j}$ imply anti/ferromagentic behaviour. The $\mathrm{H}-\mathrm{He}$ values are all small, particularly in the triplets, favoring the local magnetic image, and negative. This is important, since in the triplets each $\mathrm{He}, \mathrm{H}$ pair couples very weakly and antiferromagnetically, as it would be expected from standard weak chemical bonding. ${ }^{32}$ It also means that all ferromagnetic coupling in the triplets lies between the $\mathrm{H}$ atoms, again supporting a localized picture. Finally, we comment on the $\boldsymbol{S}_{\mathrm{H}} \cdot \boldsymbol{S}_{\mathrm{H}}$ values. Admitting the Heisenberg picture, with $H=-J_{\mathrm{HH}} \boldsymbol{S}_{\mathrm{H}} \cdot \boldsymbol{S}_{\mathrm{H}}, \Delta E_{S, T}$ would arise from $-0.773 J$ in the linear and from $-0.813 J$ in the bent configurations. This is also worthwhile exploring in the future. 


\section{Are the bridge's electrons needed at all?}

The real space picture we have just detailed provides a physical explanation of the magnetic coupling phenomenology that is dealt with, for instance, the HTH model. Moreover, real space reasonings lack the problems associated to orbital arbitrariness, although they are many times criticized for their lack of predictivity. We now show that they can lead to truly new insights.

As it has been shown, the basic role of the atomic bridge is to impose a Pauli barrier to the presence of electrons, leading to a $b_{1}^{2}$ instead of $a_{1}^{2}$ preferred configuration in linear geometries. Chemical interaction with the barrier enhances the $J$ values, but is not essential in the singlet-triplet preference, i.e. in determining the sign of $J$. It is thus strongly suggested by our findings that any object that is able to divert electrons from invading it, will elicit the observed behavior when it is put in between the two magnetic centers. This hypothesis is easily verified if we use a pseudopotential or effective core potential (ECP) to simulate the bridge. In our case it leads to a two-electron system, so that to circumvent the Lieb-Mattis theorem, ${ }^{36}$ we must choose a non-local potential, for instance one that includes non-local projection operators of the $|\phi\rangle\langle\phi|$ form. We have chosen a standard He core ECP with $p$ and $s-p$ components (see the SI for details) to simulate our systems again. Both at the 6-311G(p) and STO-6G FCI levels we reproduce the singlet to triplet ground state switching as we bend the system. We have found this to be also the case in other systems examined, like in the Li-Be-Li moiety. Table 4 summarizes the results. Notice that the ECP is absorbed as a one-electron contribution. $\Delta E_{S, T}=-24.1$ and $+4.3 \mathrm{~m} E_{h}$ for the $\mathrm{L}$ and $\mathrm{B}$ geometries, quite consistent with the global FCI values already presented.

As shown, the substitution of the He moiety by an effective non-local potential does not alter the picture much, as expected from the overall good performance of ECPs in modeling the electron structure of molecules. Grossly speaking, much as the main job of standard ECPs is to avoid the variational collapse of valence electrons over the cores by maintaining the nodal structure of the former, here the He ECP avoids invasion of the He 
Table 4: 6-311G(p) FCI data for the linear (L) and bent (B) H-V-H geometries described in the text in the presence of an ECP modeling the He atom, represented as V. S stands for singlet and $\mathrm{T}$ for triplet, and $\phi$ is the same $a_{1}$ or $b_{1}$ function as that written explicitly in the determinant for the singlets, while it is the other one in the triplets. $n$ is the occupation number of the $2 a_{1}$ and $1 b_{1}$ active natural orbitals. K, ne, ee are the total kinetic, nucleuselectron, and electron repulsion energies, and $\epsilon$ the canonical orbital energies. ECP is the one-electron expectation value of the effective core potential. All data in au.

\begin{tabular}{c|rr|rr}
\hline & LS & LT & BS & BT \\
\hline $\mathrm{E}$ & -0.8928 & -0.8687 & -0.8669 & -0.8712 \\
$\mathrm{~K}$ & 1.1799 & 1.2416 & 1.2499 & 1.2360 \\
$\mathrm{ne}$ & -2.5467 & -2.6226 & -2.7784 & -2.7614 \\
$\mathrm{ee}$ & 0.2178 & 0.2026 & 0.2734 & 0.2691 \\
$\mathrm{ECP}$ & 0.0461 & 0.0995 & 0.0945 & 0.0910 \\
$c\left(\left|a_{1} \bar{\phi}\right|\right)$ & -0.5236 & 0.7071 & 0.7094 & 0.7070 \\
$c\left(\left|b_{1} \bar{\phi}\right|\right)$ & 0.8517 & -0.7071 & -0.6467 & -0.7070 \\
$n\left(a_{1}\right)$ & 0.5483 & 0.9999 & 1.0327 & 0.9997 \\
$n\left(b_{1}\right)$ & 1.4509 & 0.9999 & 0.9669 & 0.9997 \\
$\epsilon\left(a_{1}\right)$ & -0.1654 & -0.3925 & -0.2499 & -0.4609 \\
$\epsilon\left(b_{1}\right)$ & -0.0078 & -0.4826 & -0.0022 & -0.4336 \\
\hline$\rho_{\text {bcp* }}$ & - & - & 0.0141 & 0.0147 \\
$\nabla^{2} \rho_{\text {bcp* }}$ & - & - & 0.0536 & 0.0577 \\
\hline$E_{\text {self }}^{\mathrm{H}}$ & -0.4630 & -0.4826 & -0.4752 & -0.4746 \\
$E_{\text {int }}^{\mathrm{HH}}$ & -0.0129 & -0.0031 & -0.0108 & -0.0131 \\
\hline$\delta^{\mathrm{HH}}$ & 0.1455 & 0.0277 & 0.0722 & 0.0837 \\
\hline$p(2,0)$ & 0.0364 & 0.0070 & 0.0181 & 0.0209 \\
\hline$S_{\mathrm{H}}^{2}$ & 0.6954 & 0.7395 & 0.7228 & 0.7187 \\
\hline
\end{tabular}


nuclear region, reinforcing our points above. Since most of the descriptors in Table 4 follow the same trends as those already discussed in Tables 1-3, we will only comment on a few of them. On the one hand, the majority of ECP orbital descriptors evolve as in the full electron calculation, particularly the kinetic and electron repulsion energies, as well as the $a_{1}$ and $b_{1}$ natural occupations. The large $b_{1}^{2}$ contribution in the LS lies again behind its large $\delta^{\mathrm{HH}}$ value. Similarly, as in the full electron calculation, the LT system displays the lowest ionic contribution. On the other hand, the total FCI energies excluding the ECP one-electron potential contributions favor the triplet in both the linear and angular configuration. The ECP row of Table 4 shows that the destabilizing effect of the pseudopotential is about 0.05 au lower in the LS than in any of the LT, BS, or BT geometries, in line with the stabilizing singlet contributions of the HTH model.

Finally, it is noteworthy that in the absence of the perturbing effect of the He electrons, the traditional correlation between stabiliy and density at the critical point is also found in the bent configuration. The more stable triplet leads to the largest $\rho_{b c p} *$. This has been found quite general in several cases, see below.

\section{Tuning the coupling in real space}

Using the insights derived above regarding the physical role of the bridge, it is illuminating to examine how the singlet-triplet gaps evolve when the nature of the bridge is modified. This can be done without leaving the toy models we have explored so far. We briefly review the outcome of: a) substituting the He closed-shell barrier by an absorbing $\mathrm{He}^{2+}$ potential; b) allowing for the electrons of the barrier to significantly mix with the magnetic states by changing $\mathrm{He}$ by a $\mathrm{Be}^{2+}$ core c) using a more realistic Li-Be-Li triatomic system to show how the results resemble those of the the H-He-H example. The full data can be found in the supplementary information.

Removing the He core while maintaining the linear and bent geometries already examined 


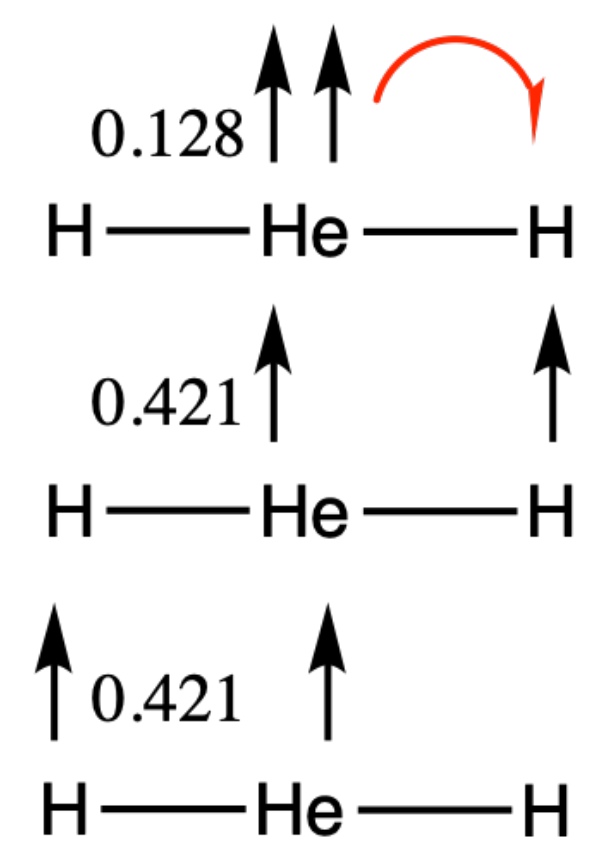

Figure 6: FCI/6311-G(p) dominant probability distribution of the LT state of $\mathrm{H}-\mathrm{He}-\mathrm{H}^{2+}$ at $R_{\mathrm{HHe}}=1.2587 \AA$.

provides a very deep attractive potential to the electrons residing in the magnetic centers, since the orbital energy of the He 1s orbital is about -0.917 au. This means that a huge electronegativity difference exists that forces the electrons to invade the He region. Since the orbital energy of the $2 a_{1}$ function is more negative than that of the $1 b_{1}$ one in the LT and BT states, both prefer a predominant $1 a_{1} 2 a_{1}$ configuration that maximizes the now allowed delocalization. As the LS and BS states are regarded, they can be considered distorted He atoms, with very small $\mathrm{H}$ atomic basins (hosting about 6 me). Their FCI wavefunctions are fully dominated by the $\mathrm{HF} 1 a_{1}^{2}$ configuration, with coefficients larger than 0.995. In the linear configuration, $\Delta E_{S, T}=-402.5 \mathrm{kcal} / \mathrm{mol}$ at the FCI $/ 6-311(\mathrm{p})$ level. Bending does not alter the picture and the gap is now $-369.7 \mathrm{kcal} / \mathrm{mol}$. However, since $\Delta E_{S, T}=-689.6 \mathrm{kcal} / \mathrm{mol}$ for the He atom at the current FCI level, a considerable lowering of the triplet energy is obtained by delocalizing into the $\mathrm{H}$ basins, which now host $0.451 e$ each in the LT configuration. Its probability distribution of finding electrons in the $\mathrm{H}, \mathrm{He}, \mathrm{H}$ basins, respectively is dominated by three contributions: $p(1,1,0)=p(0,1,1)=0.421$ and $p(0,2,0)=0.128$, with a considerable $\mathrm{H}-\mathrm{He}$ delocalization index of 0.148 , and a remarkable 
$\mathrm{H}-\mathrm{H}$ one rising to 0.354 , see Fig. 6. This shows how the pure He triplet is avoided. A local spin analysis (SI) shows that the spin coupling between the two $\mathrm{H}$ regions is essentially negligible (even slightly antiferromagnetic, $\left\langle\boldsymbol{S}_{\mathrm{H}} \cdot \boldsymbol{S}_{\mathrm{H}}\right\rangle \approx-0.0009$ ). This example offers a global picture of atomic-like singlets and delocalized triplets, very far from the isolated magnetic centers already discussed. In real space, removing the Pauli barrier fosters delocalization, and this is much more efficient in the singlets, irrespective of the geometry.
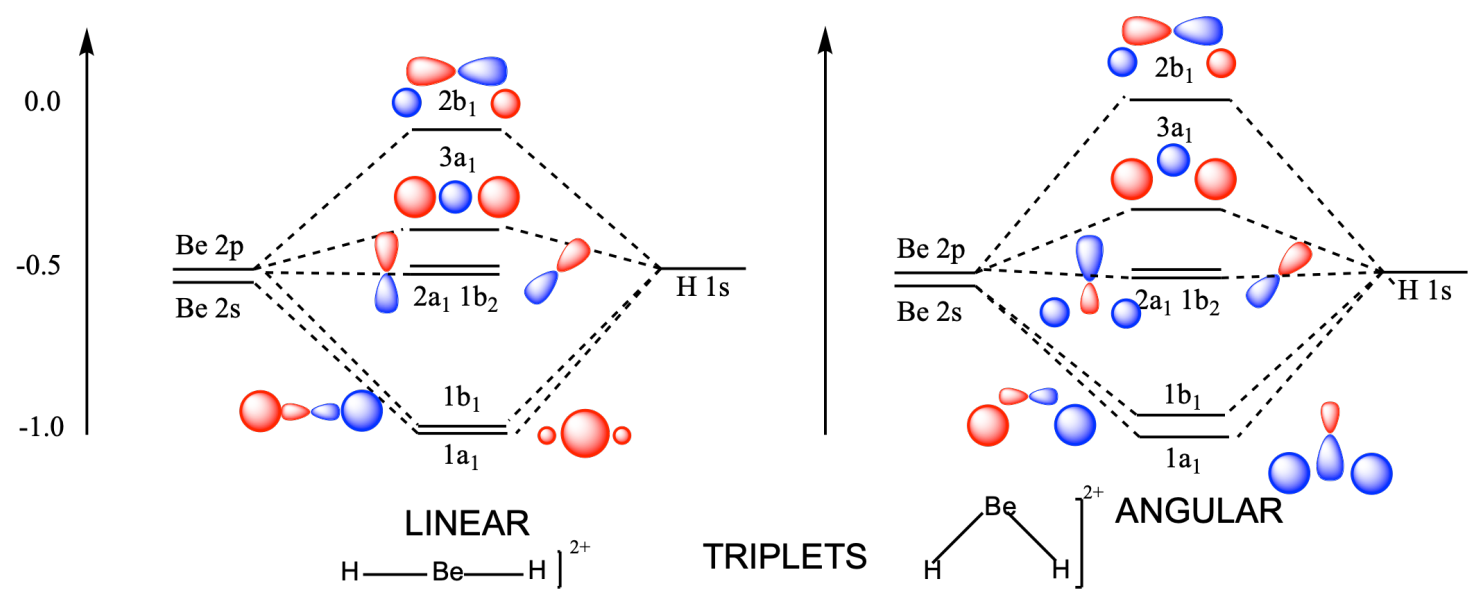

Figure 7: STO-6G orbital diagram for the linear and bent triplet states of $\mathrm{H}-\mathrm{Be}-\mathrm{H}^{2+}$ in both the linear and bent geometries. The canonical orbital energies have been drawn approximately on an energy scale in au, and the Be 1s core has been excluded.

The scenario changes completely if the Pauli barrier is substituted by available states that can mix strongly with the magnetic centers. This is easily done by introducing a $\mathrm{Be}^{2+}$ bridge, with $\epsilon_{2 s, 2 p} \approx-0.665,-0.519$ au, respectively, when a 6-311G(p) basis is used. At the fixed linear and bent geometries examined, the FCI $\Delta_{S, T}$ are +243 and $-11724 \mathrm{~cm}^{-1}$, respectively, so now the triplet is the ground state in the linear geometry. As in our main example, this ordering is stable even at the STO-6G level. Fig. 7 shows the qualitative orbital diagram for the two triplet states. The BS falls well below the BT thanks to the polarizable nature of the Be states (the presence of available $2 \mathrm{p}$ functions in orbital parlance). The dominant $1 a_{1}^{2}$ coefficient in the BS CI expansion is 0.922 , showing that the H-He-H constraints forcing the BS to resemble the Coulson-Fischer singlet have now disappeared. Interestingly, it is now the orbital energies of the $1 a_{1}, 1 b_{1}$ states in the LS that are extremely 


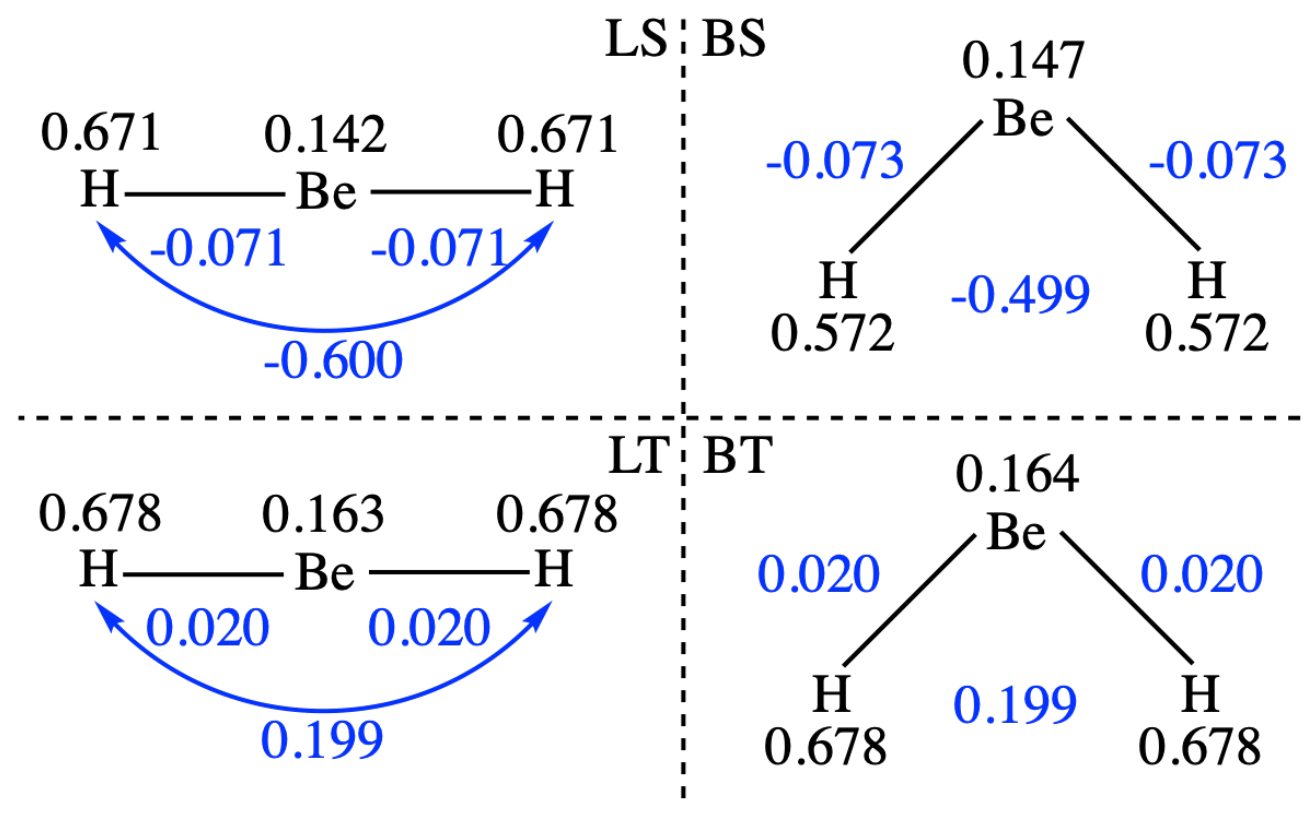

Figure 8: FCI/6311-G(p) local spin analysis for the H-Be-H ${ }^{2+}$ system. $\left\langle S_{A}^{2}\right\rangle$ values in black, $\left\langle\boldsymbol{S}_{A} \cdot \boldsymbol{S}_{B}\right\rangle$ couplings in blue.

close, $-0.981,-0.974 \mathrm{au}$, respectively. This allows the HTH expression to predict a possible triplet ground state. Now the physical root of this behavior is the considerably larger kinetic energy of the $\phi_{1 a_{1}}$ with respect to $\phi_{1 b_{1}}$, at variance with the He barrier. Although the $b_{1}$ function has a nodal plane, its He contribution is 2p-like, with a considerably smaller kinetic energy than the 2 s one. This effect is now the driving force that gives rise to a strong singlet mixing, $\Psi_{\mathrm{BS}} \approx 0.722\left|1 a_{1} 1 \bar{a}_{1}\right|-0.690\left|1 b_{1} 1 \bar{b}_{1}\right|$, switching on the Coulson-Fischer behavior of the singlet. All real space descriptors show that the triplet should be preferred. For instance, $\delta^{\mathrm{HH}}=0.0177,0.0180$ and $\delta^{\mathrm{HHe}}=0.186,0.188$ for the LS and LT states, respectively. Almost all of the linear singlet-triplet gap of $0.69 \mathrm{kcal} / \mathrm{mol}\left(1.1 \mathrm{mE}_{h}\right)$ can be ascribed to a change in the self-energy of the Be atom, which passes from -13.7574 to -13.7588 au on going from the LS to the LT. The local spin analysis (Fig. 8) shows very clearly that the localized magnetic centers picture applies rather well in the LS (singlet diradical), LT, and BT cases, and that clear signs of $\mathrm{H}-\mathrm{H}$ bonding appear in the BS case, since the $\mathrm{H}$ atom local spin is now considerably quenched, and the $\mathrm{H}-\mathrm{H}$ singlet coupling decreased, with respect to the LS state. This is corroborated by examining the delocalization indices and the distribution 


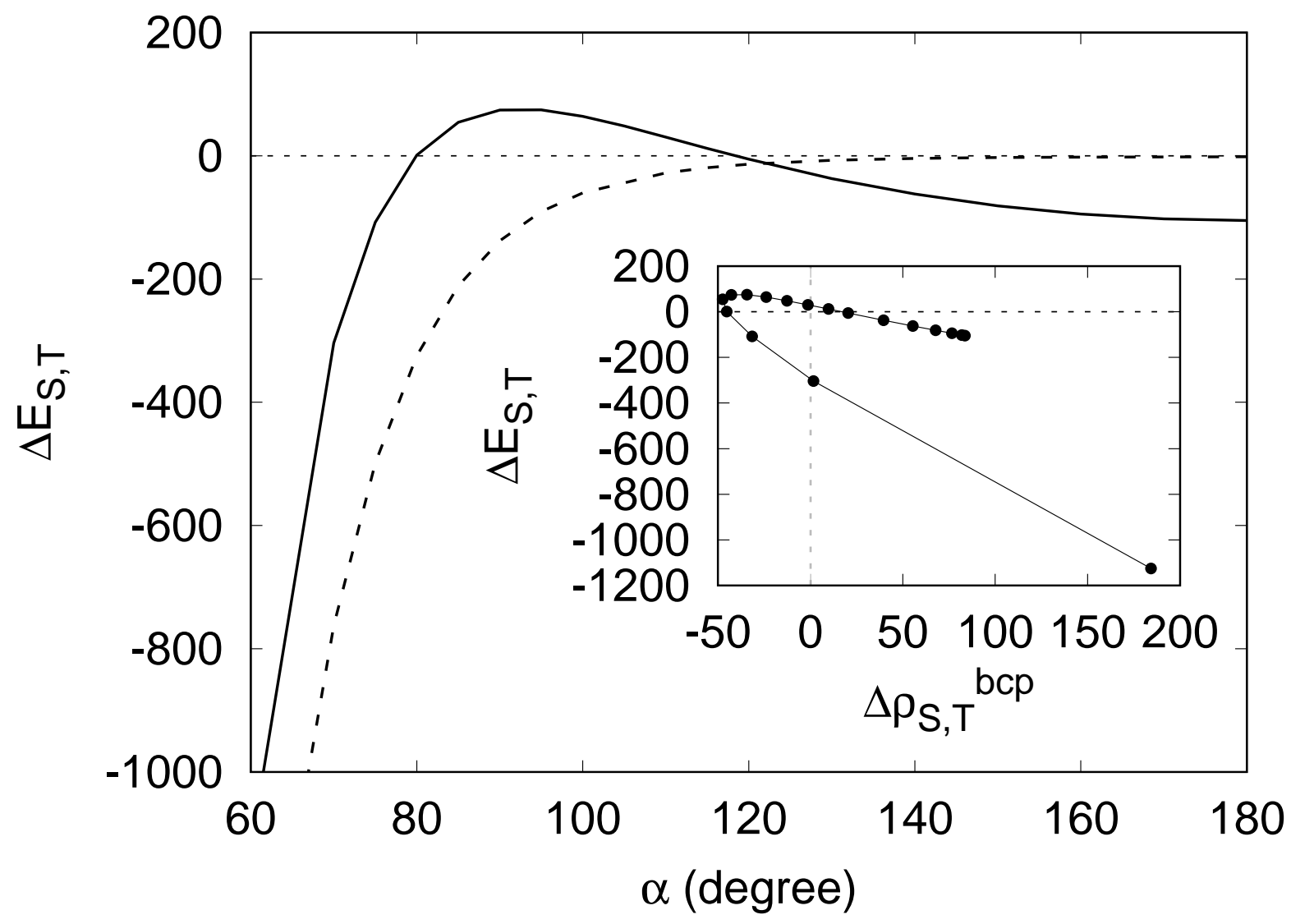

Figure 9: Singlet-Triplet splitting in $\mathrm{Li}_{2} \mathrm{Be}$ (dashed line). The bare $\mathrm{Li}_{2}$ behavior is also included for comparison (solid line). The inset shows the $\Delta E_{S, T}-\Delta \rho_{S, T}^{b c p}$ correlation in the weak coupling regime (upper branch) and in the $\mathrm{Li}_{2}$ molecule regime (lower branch). Energies in $\mathrm{cm}^{-1}$ and densities in $\mu e$.

function probabilities. A polarizable (in contrast to a rigid) atomic bridge with shallow available states provides a triplet linear ground state with interesting real space branches.

To show that our real space arguments can be applied to similar cases, we show the weak interacting behavior of the Li-Be-Li system at $R_{\mathrm{LiBe}}=8.00$ au as we change the $\alpha=\widehat{\mathrm{LiBeLi}}$ angle. In the weak-coupling region a $\mathrm{CAS}[4,7] / / 6-311+\mathrm{G}^{* *}$ wavefunction is of sufficient quality. We only consider how the change in the density at the Li-Be bond critical point evolves with the singlet-triplet gap, shown in Fig. 9. Notice that we can again classify the Be bridge as a Pauli barrier, so that we expect the same behavior as in $\mathrm{H}-\mathrm{He}-\mathrm{H}$. The bridge clearly amplifies the gap, and provides a triplet ground state at intermediate angles. $\Delta \rho$ at the Li-Be bcp's is a good predictor of $\Delta E_{S, T}$ : the state that accumulates more electron 
density in the Li-Be bonding region is the most stable. The $\Delta E_{S, T}$ versus $\Delta \rho_{b c p}$ curve has two branches: one corresponding to the low angle regime, and another one to the high angle one. The spatial antisymmetry of the triplet is again important to explain the angular dependence of it energy. This state must show a slight density depletion at the Li-Li midpoint, which necessarily means a charge buildup out of the Li-Li axis. For $\alpha$ angles smaller than about 120 degrees, such accumulation lies well into the Li-Be bonding region, and the triplet falls below the singlet. At smaller angles the Li-Li distance decreases, and an incipient Li-Li bond forms so that density starts to accumulate again in between the lithiums, and the Li-Li bcp takes over the Li-Be bond, which ceases to determine the behavior of the system (the lower branch of the inset). In this region, the singlet's energy falls quickly, approaching the curve of the bare $\mathrm{Li}_{2}$.

\section{Conclusions}

Physically understanding the roots of the coupling between magnetic centers in the simplest molecular systems is of utmost importance. This knowledge is used to build the models with which we describe the collective magnetic behavior of more complex entities, that ultimately leads to the design and synthesis of novel functional magnetic materials. The problem has been attacked since the earliest days of quantum mechanics and, after the development of modern computational techniques, it is now possible to predict, sometimes even quantitatively, magnetic coupling constants in complex materials ab initio. When the simplest two magnetic centers case is examined, molecular orbital theory led to the Hay, Thilbeault, and Hoffmann (HTH) model, which relies in the use of orthogonal localized magnetic orbitals. The HTH model has become the qualitative paradigm used to interpret the origins of singlet-triplet gaps, characterized by a direct ferromagnetic contribution given by the exchange integral $K_{a b}$ between the magnetic orbitals, and a counteracting antiferromagnetic term rooted in the larger variational flexibility of the singlet state, that implies a magnetic 
exchange mediated by ionic contributions. It also provides the basic ingredients to rationalize the singlet-triplet preference (an example of the so-called magnetostructural correlations) in simple dinuclear metallic complexes.

Successful as it has been, the HTH (or its equivalent Kahn-Briat variant) paradigm rests on a given choice of orthogonal one-electron functions out of an infinitely many set of possibilities. For instance, both the direct exchange as well as the ionic antiferromagnetic contributions can be manipulated, almost at will, by performing unitary rotations of the orbital space. It is then very relevant to examine the problem from an orbital invariant perspective, as that offered by real space analyses. We have concentrated on the very simplest two-center cases that can be imagined, the dihydrogen molecule and the H-He-H system, which already shows a singlet to triplet switch as we bend the $\mathrm{H}-\mathrm{He}-\mathrm{H}$ angle.

We have first shown that it is electron delocalization that drives any singlet-triplet gap and any ionic mixing. It is shown that delocalization and ionic mixing are two sides of the same coin, so that if the HTH paradigm can be restated in an orbital invariant framework, electron delocalization measures should play a major role. By examining the behavior of the H-He-H system using both orbital arguments and then real space ones, we demonstrate that it is the Pauli barrier introduced by the atomic bridge that crafts the electronic structure of the system. It does so by driving the system to choose the antisymmetric linear combination of the H orbitals instead of the normal symmetric one, in an effort to decrease the electron's kinetic energy. In the linear structure, configuration mixing allows the system to come to a beneficial compromise. Bending leads to a new energetic balance that can only be achieved by letting the singlet electron density resemble that of the triplet. In doing this, its advantage is lost.

Second, we have demonstrated that delocalization, allowed or prevented by the different symmetry of the wavefunctions of singlets and triplets, lies behind the preferred coupling mode. In this sense, a real space picture provides direct access to this concept. The systems behave as diradial species, although a non-negligible amount of spin is transferred to the 
bridging atom. As noticed, local spin analyses might have an impact on how state-of-the-art calculations can be used to obtain $J$ coupling constants, and deserve further consideration. Similarly, local energy techniques demonstrate that although the bridge amplifies the stabilization of the singlet in the linear geometry, as it is well known from the HTH model, all the qualitative features including the singlet-triplet preference lie within the subsystem formed by the two magnetic atoms. The amplification of the singlet stabilization induced by the bridge can be measured by the metal-bridge interaction energy, which makes full chemical sense. A number of bridge-magnetic center $(\mathrm{He}-\mathrm{H})$ real space interaction descriptors (bond critical point densities, interaction energies, etc) correlate with the singlet-triplet gap. We have also shown that exchange pathways can be clearly sensed by studying the probabilities of different electron distributions, all of them orbital invariant.

Finally, our findings have pointed toward the non-essential role of the atomic bridge's electrons in setting up the magnetic coupling. To check this idea, we have substituted the bridge by an effective core potential. Not unexpectedly, the absence of the bridge's electrons does not alter the singlet-triplet ordering, showing that any object that mimics appropriately the Pauli barrier of the bridge will induce the correct sign of the singlet-triplet gap. As shown, the nature of the wavefunctions of the singlet and triplet states, as analyzed in real space, follows closely the all-electron results.

Tuning the singlet-triplet gap is also possible using real space thinking. We have shown how this is modified examining three simple cases where the bridge is transformed into an electron absorbing center at two different energies and when a more realistic system, Li-Be-Li is examined.

Real space analyses thus question the accepted magnetic coupling paradigm based on orbital thinking from several different angles. We believe that new avenues can be opened by using these orbital invariant techniques to understand magnetic behavior that may be not obvious from other perspectives. 


\section{Acknowledgments}

We thank the Spanish MINECO, grant PGC2018-095953-B-I00 and the European Union FEDER for funding.

\section{References}

(1) Mattis, D. The theory of magnetism made simple: an introduction to physical concepts and to some useful mathematical methods; World Scientific: Singapore Hackensack, NJ, 2006.

(2) Uhlenbeck, G. E.; Goudsmit, S. Nature 1926, 117, 264-265.

(3) Dirac, P. A. M. Proceedings of the Royal Society of London. Series A, Containing Papers of a Mathematical and Physical Character 1928, 117, 610-624.

(4) Vleck, J. The theory of electric and magnetic susceptibilities; Oxford University Press: New York, 1932.

(5) Stoner, E. C. Magnetism and Matter; Methuen: London, 1934.

(6) Dirac, P. A. M. Proceedings of the Royal Society of London. Series A 1926, 112, 661677.

(7) Heisenberg, W. Zeitschrift für Physik 1926, 38, 411-426.

(8) Ising, E. Zeitschrift für Physik 1925, 31, 253-258.

(9) Slater, J. C. Reviews of Modern Physics 1953, 25, 199-210.

(10) Kahn, O. Molecular magnetism; VCH: New York, NY, 1993.

(11) Kahn, O.; Briat, B. J. Chem. Soc., Faraday Trans. 2 1976, 72, 268-281.

(12) Shull, C. G.; Smart, J. S. Physical Review 1949, 76, 1256-1257. 
(13) Anderson, P. W. Physical Review 1950, 79, 350-356.

(14) Kramers, H. Physica 1934, 1, 182-192.

(15) Goodenough, J. B. Physical Review 1955, 100, 564-573.

(16) Kanamori, J. Journal of Physics and Chemistry of Solids 1959, 10, 87-98.

(17) Hay, P. J.; Thibeault, J. C.; Hoffmann, R. Journal of the American Chemical Society 1975, 97, 4884-4899.

(18) Cabrero, J.; de Graaf, C.; Bordas, E.; Caballol, R.; Malrieu, J.-P. Chemistry - A European Journal 2003, 9, 2307-2315.

(19) Loth, P. D.; Cassoux, P.; Daudey, J. P.; Malrieu, J. P. Journal of the American Chemical Society 1981, 103, 4007-4016.

(20) Rodriguez, J. H.; McCusker, J. K. The Journal of Chemical Physics 2002, 116, 62536270.

(21) Malrieu, J. P.; Caballol, R.; Calzado, C. J.; de Graaf, C.; Guihéry, N. Chemical Reviews 2013, 114, 429-492.

(22) Calzado, C. J.; Cabrero, J.; Malrieu, J. P.; Caballol, R. The Journal of Chemical Physics 2002, 116, 2728-2747.

(23) Calzado, C. J.; Cabrero, J.; Malrieu, J. P.; Caballol, R. The Journal of Chemical Physics 2002, 116, 3985-4000.

(24) de Graaf, C.; Broer, R. Magnetic interactions in molecules and solids; Springer: Heidelberg, Germany, 2016.

(25) Shaik, S. A chemist's guide to valence bond theory; Wiley-Interscience: Hoboken, N.J, 2008. 
(26) Martín Pendás, A.; Francisco, E. Phys. Chem. Chem. Phys. 2018, 20, 12368-12372.

(27) Bader, R. F. W. Atoms in molecules : a quantum theory; Clarendon Press: Oxford, 1990.

(28) Blanco, M. A.; Martín Pendás, A.; Francisco, E. Journal of Chemical Theory and Computation 2005, 1, 1096-1109.

(29) Francisco, E.; Martín Pendás, A.; Blanco, M. A. J. Chem. Theory Comput. 2006, 2, $90-102$.

(30) Francisco, E.; Martín Pendás, A.; Blanco, M. A. Computer Physics Communications 2008, 178, $621-634$.

(31) Francisco, E.; Martín Pendás, A.; Blanco, M. A. Journal of Chemical Physics 2007, 126, 094102-1-094102-13.

(32) Martín Pendás, A.; Francisco, E. Physical Chemistry Chemical Physics 2021, 23, 83758392.

(33) Shaik, S.; Danovich, D.; Wu, W.; Hiberty, P. C. Nat. Chem. 2009, 1, 443-449.

(34) Bader, R. F. W.; Stephens, M. E. Chem. Phys. Lett. 1974, 26, 445-449.

(35) Outeiral, C.; Vincent, M. A.; Martín Pendás, A.; Popelier, P. L. A. Chemical Science 2018, 9, 5517-5529.

(36) Lieb, E.; Mattis, D. Physical Review 1962, 125, 164-172.

(37) Earnshaw, S. Trans. Camb. Phil. Soc., 1842, 7, 97-112.

(38) Francisco, E.; Martín Pendás, A.; García-Revilla, M.; Boto, R. Á. Computational and Theoretical Chemistry 2013, 1003, 71 - 78, Reduced Density Matrices: A Simpler Approach to Many-Electron Problems? 
Questioning the orbital picture of magnetic spin coupling: a real space alternative

Electronic Supplementary Information.

A. Martín Pendás, E. Francisco

July 27, 2021 


\section{Contents}

$1 \quad$ Real Space Chemical bonding in a nutshell 3

1.1 The energetic face of bonding: Interacting Quantum Atoms . . . . . . . . . . . 3

1.2 The electron-counting face of bonding: Electron Distribution Functions . . . . . 4

1.3 Ionic and covalent structures in $2 \mathrm{c}-2 \mathrm{e}$ bonds . . . . . . . . . . . . . . . . 5

1.4 Local spin . . . . . . . . . . . . . . . . . . . . . 6

2 The Coulson-Fischer state and its real space analysis $r$

$\begin{array}{llr}3 & \text { Methodological details } & 9\end{array}$

4 Raw Results 10

$4.1 \mathrm{H}-\mathrm{He}-\mathrm{H} \mathrm{LS} \ldots \ldots \ldots \ldots \ldots \ldots \ldots$

$4.2 \mathrm{H}-\mathrm{He}-\mathrm{H} \mathrm{LT} \ldots \ldots \ldots \ldots \ldots \ldots$

$4.3 \mathrm{H}-\mathrm{He}-\mathrm{H} \mathrm{BS} \ldots \ldots \ldots \ldots \ldots \ldots \ldots$

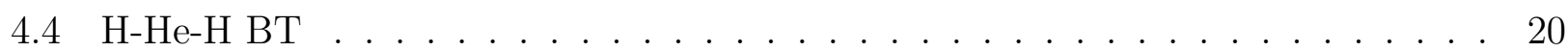

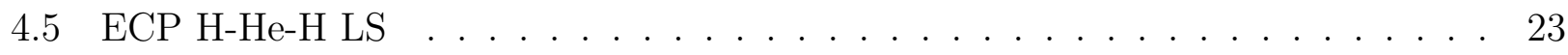

4.6 ECP H-He-H LT . . . . . . . . . . . . . . . . . . . . . . . . . . . . 24

4.7 ECP H-He-H BS . . . . . . . . . . . . . . . . . . . 25

4.8 ECP H-He-H BT . . . . . . . . . . . . . . . . . . . 26

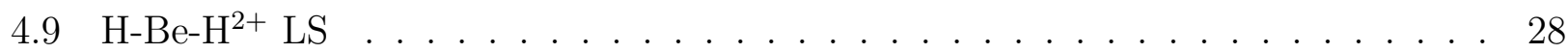

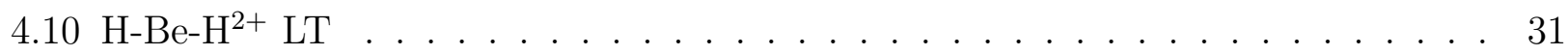

$4.11 \mathrm{H}-\mathrm{Be}-\mathrm{H}^{2+} \mathrm{BS} \ldots \ldots \ldots \ldots \ldots \ldots \ldots \ldots$

$4.12 \mathrm{H}-\mathrm{Be}-\mathrm{H}^{2+} \mathrm{BT} \ldots \ldots \ldots \ldots \ldots \ldots \ldots$

$4.13 \mathrm{H}-\mathrm{He}-\mathrm{H}^{2+} \mathrm{LS} \ldots \ldots \ldots \ldots \ldots$

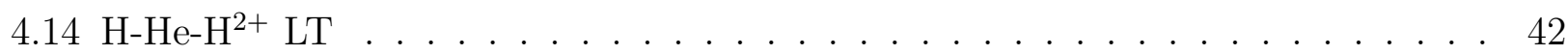

$4.15 \mathrm{H}-\mathrm{He}-\mathrm{H}^{2+} \mathrm{BS} \ldots \ldots \ldots \ldots \ldots \ldots \ldots \ldots$

$4.16 \mathrm{H}-\mathrm{He}-\mathrm{H}^{2+} \mathrm{BT} \ldots \ldots \ldots \ldots \ldots \ldots \ldots$

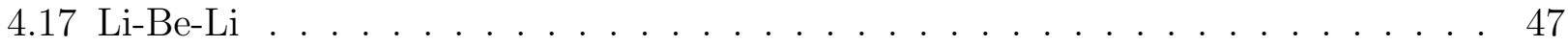




\section{Real Space Chemical bonding in a nutshell}

Real space reasoning uses proper quantum mechanical observables to construct orbital invariant descriptors with chemical meaning. Among them, all reduced densities and density matrices (RDs, RDMs). An identification of spatial regions with chemical concepts is also necessary. This is usually done through spatial partitionings, normally induced by the topology of a scalar field. For instance, the topology of the electron density, $\rho$, induces an atomic partitioning: the Quantum Theory of Atoms in Molecules (QTAIM) explored by R. F. W. Bader and coworkers. ${ }^{1}$ Similarly, the topology of Becke and Edgecombe's ${ }^{2}$ electron localization function (ELF) provides a partition into cores, lone pairs and bonding domains, etc.

Once atoms (or electron-pair domains) are available, chemical bonding descriptors are built. Both the electron-counting perspective (leading to populations and bond orders) as well as the energetic view that provides bond strengths are needed. These are offered by, for instance, electron distribution functions (EDFs) and the interacting quantum atoms approach (IQA).

\subsection{The energetic face of bonding: Interacting Quantum Atoms}

Given an atomic spatial partitioning, the interacting quantum atoms (IQA) energy partition considers the one- and two-domain division of the non-relativistic Born-Oppenheimer electronic energy $^{3}$ described in the following equation,

$$
\begin{aligned}
E & =\sum_{\mathrm{A}} E_{\mathrm{self}}^{\mathrm{A}}+\sum_{\mathrm{A}>\mathrm{B}} E_{\mathrm{int}}^{\mathrm{AB}} \\
& =\sum_{\mathrm{A}} T^{\mathrm{A}}+V_{\mathrm{ne}}^{\mathrm{AA}}+V_{\mathrm{ee}}^{\mathrm{AA}}+\sum_{\mathrm{A}>\mathrm{B}} V_{\mathrm{nn}}^{\mathrm{AB}}+V_{\mathrm{ne}}^{\mathrm{AB}}+V_{\mathrm{ne}}^{\mathrm{BA}}+V_{\mathrm{ee}}^{\mathrm{AB}},
\end{aligned}
$$

wherein $E_{\mathrm{self}}^{\mathrm{A}}$ and $E_{\mathrm{int}}^{\mathrm{AB}}$ are the IQA self and interaction energies of atom $\mathrm{A}$ and pair $\mathrm{AB}$, while $T^{\mathrm{A}}$ denotes the kinetic energy of atom A. Finally, the terms $V_{\mathrm{ne}}^{\mathrm{AB}}$ and $V_{\mathrm{ee}}^{\mathrm{AB}}$ stand for (i) the attraction between the nucleus of domain $\mathrm{A}$ and the electrons of atom $\mathrm{B}$ and (ii) the repulsion between the electrons in atom $\mathrm{A}$ with those in basin $\mathrm{B}$, respectively. The self-energy of an atom is the trace of its in vacuo Hamiltonian over the atomic region it occupies in a molecule. In a process where the atoms of a system dissociate their self-energies tend to the free atomic energies.

We can get further insight about the nature of the interaction between two atoms by separating the electronic repulsion into its Coulombic and exchange-correlation components. This splitting allows, in turn, the separation of the IQA interaction energy of a pair AB as ${ }^{3}$

$$
E_{\mathrm{int}}^{\mathrm{AB}}=V_{\mathrm{cl}}^{\mathrm{AB}}+V_{\mathrm{xc}}^{\mathrm{AB}}=E_{\mathrm{ion}}^{\mathrm{AB}}+E_{\mathrm{cov}}^{\mathrm{AB}} .
$$

Usually binding is measured relative to appropriate reference for the quantum fragments $A$, with $E^{A, 0}$ energies. Then $E_{\text {self }}^{A}-E^{A, 0}=E_{\text {def }}^{A}$ is called the atomic or fragment deformation energy, which corresponds to a combination of the traditional promotion energy and other effects, like spin-recoupling, true electronic deformation, etc. ${ }^{4}$ We have shown that the IQA interaction energies behave as in situ bond energies. IQA thus provides an invariant decomposition of the energy into group deformations and bond contributions in which covalent and ionic energies acquire rather pure forms. 


\subsection{The electron-counting face of bonding: Electron Distribution Functions}

Electron counting provides access to the more qualitative view of chemical bonding in which the number of electrons engaged in sharing or in pure transfer between atoms gives rise to bonding descriptors like bond orders. In real space we simply examine the distribution of the electron population in the atomic regions in which we have divided the space.

EDFs are defined as follow. Given an $N$-electron molecule and an exhaustive partition of the real space $\left(\mathcal{R}^{3}\right)$ into $m$ arbitrary regions $\Omega_{1}, \Omega_{2}, \ldots, \Omega_{m}\left(\Omega_{1} \cup \Omega_{2} \cup \cdots \cup \Omega_{m}=\mathcal{R}^{3}\right)$, an EDF is the distribution function formed by all the probabilities $p\left(n_{1}, n_{2}, \ldots, n_{m}\right)$ of finding exactly $n_{1}$ electrons in $\Omega_{1}, n_{2}$ electrons in $\Omega_{2}, \ldots$, and $n_{m}$ electrons in $\Omega_{m},\left\{n_{p}\right\}$ being integers $\left(n_{i} \in \mathcal{N}\right)$ satisfying $n_{1}+n_{2}+\cdots+n_{m}=N$. This view is in accord with considering subsystems as open quantum systems in which number operators do not commute with the subsystem hamiltonian. In this way, $\Psi$ is not an eigenstate of the operator defining the number of electrons in domain $\Omega_{i}, \hat{N}_{\Omega_{i}}$. This means that the average number of electrons in $\Omega_{i}$ is not an eigenvalue of $\hat{N}_{\Omega_{i}}$, so that measuring the number of electrons in the domain will render values $n_{\Omega_{i}}$ ranging from 0 to $N$, the total number of electrons, with a defined set of probabilities, $p\left(n_{\Omega_{1}}\right)$. This is the one-fragment EDF for domain $\Omega_{i}$. To obtain these probabilities or, in general, the multivariate electron distribution functions $p\left(n_{1}, n_{2}, \cdots, n_{m}\right)$, one needs $\Psi(1, \ldots, N), \Psi$ being the complete wave function,

$$
p\left(n_{1}, n_{2}, \ldots, n_{m}\right)=N ! \Lambda \int_{D} \Psi^{\star} \Psi d \mathbf{x}_{1} \cdots d \mathbf{x}_{N},
$$

where $D$ is a multidimensional domain in which the first $n_{1}$ electrons are integrated over $\Omega_{1}$, the second $n_{2}$ electrons over $\Omega_{2}, \cdots$, and the last $n_{m}$ electrons over $\Omega_{m}$, and $N ! \Lambda=$ $N ! /\left(n_{1} ! n_{2} ! \cdots n_{m} !\right)$ is a combinatorial factor that accounts for electron indistinguishability. The 3D domains of these integrations can be arbitrary, but when using QTAIM atomic basins, a partition of the $N$ electrons of the molecule that assigns a given number of electrons (including possibly 0) to each of these regions will was called a real space resonance structure (RSRS $)^{5}$ and there are $N_{S}=(N+m-1) ! /[N !(m-1) !]$ of these for a given $N, m$ pair. With the notation $S\left(n_{1}, n_{2}, \ldots, n_{m}\right) \equiv S\left(\left\{n_{p}\right\}\right)$, or simply $\left(n_{1}, n_{2}, \ldots, n_{m}\right) \equiv\left\{n_{p}\right\}$, we label the resonance structure having $n_{1}$ electrons in $\Omega_{1}, n_{2}$ electrons in $\Omega_{2}, \ldots$, and $n_{m}$ electrons in $\Omega_{m}$. If electrons are spin-seggregated, then we come to spin-resolved EDFs, and a set of probabilities $p\left(n_{1}^{\alpha}, n_{1}^{\beta}, n_{2}^{\alpha}, n_{2}^{\beta}, \ldots, n_{m}^{\alpha}, n_{m}^{\beta}\right)$ which gives extremely fine-grained information about how electrons and their spins distribute. ${ }^{6}$

The computation of $p\left(n_{1}, n_{2}, \ldots, n_{m}\right)$ for all the RSRSs provides all the statistical moments of the electron populations, including the average number of electrons in a given region, or its fluctuation. The average population is obviously given by

$$
N_{i}=\left\langle n_{i}\right\rangle=\sum_{\left\{n_{p}\right\}} n_{i} \times p\left(\left\{n_{p}\right\}\right)=\sum_{n_{i}} n_{i} p_{i}\left(n_{i}\right) .
$$

It is not difficult to show that the number of shared pairs between two regions may be obtained directly by counting the number of intra- and interpairs. ${ }^{7}$ This has given rise to the so-called localization and delocalization indices, $\left(\lambda^{i i}, \delta^{i j}\right)$, which determine the number of localized and delocalized pairs. The latter, which is the covalent bond-order in real space can be obtained from the $p\left(\left\{n_{p}\right\}\right)$ probabilities as 


$$
\begin{array}{r}
\delta^{i j}=-2 \operatorname{cov}(i, j)=-2\left[\left\langle n_{i} n_{j}\right\rangle-\left\langle n_{i}\right\rangle\left\langle n_{j}\right\rangle\right]= \\
-2\left[\sum_{\left\{n_{p}\right\}} n_{i} n_{j} \times p\left(\left\{n_{p}\right\}\right)-\left\langle n_{i}\right\rangle\left\langle n_{j}\right\rangle\right]= \\
-2 \sum_{n_{i} n_{j}}\left(n_{i}-N_{i}\right)\left(n_{j}-N_{j}\right) p\left(n_{i}, n_{j}\right)=2 N_{i j}
\end{array}
$$

where the -2 factor has been included to comply with the usual definition of $\delta$ in terms of the exchange-correlation density and to ensure that the bond order for an ideal single bond is equal to 1 ,

$$
\delta^{i j}=-2 \int_{\Omega_{i}} \int_{\Omega_{j}} d \mathbf{1} d \mathbf{2} \rho_{x c}(1,2) .
$$

The localization index is given by

$$
\lambda_{i i}=N_{i}-\operatorname{cov}(i, i)=N_{i}-\operatorname{var}(i)=N_{i}-\sum_{n_{i}}\left(n_{i}-N_{i}\right)^{2} p\left(n_{i}\right)=N_{i i}
$$

From equations 5-9 it is clear that $N_{i i}=N_{i}$ if the variance is zero and that $N_{i j}=0$ if the covariance is zero. This is the starting point for a complete theory of chemical bonding based on the fluctuation of electron populations. There is chemical bonding between two regions if their electron populations are not statistically independent. A sum rule, that classifies electrons into localized and delocalized sets appears:

$$
N=\sum_{\Omega_{i}} N_{i}=\sum_{\Omega_{i}} \lambda^{i i}+\frac{1}{2} \sum_{\Omega_{i} \neq \Omega_{j}} \delta^{i j} .
$$

Suitable generalizations in the case of multi-center bonding exist. ${ }^{8}$

\subsection{Ionic and covalent structures in $2 \mathrm{c}-2 \mathrm{e}$ bonds}

The statistical link between the fluctuation of electron populations and the standard energetic and bond order descriptors allows to map all coarse-grained (i.e. condensed at the atomic level) possible $(2 c-2 e)$ bonds through simple models. In a two-center, two-electron system there are only three RSRSs: $(2,0),(1,1),(0,2)$, where we label how many electrons lie in each of the $a, b$ domains. The central structure is obviously identified with the valence-bond covalent structure, while the other two describe ionic distributions. The EDF space is two-dimensional, since $p(2,0)+p(1,1)+p(0,2)=1$, and all bond indices become fully mapped in this $2 \mathrm{D}$ space. A convenient coordinate system can be built with the probability that any of the electrons lie in one of the basins, e.g. the left one, which we call $p$ and provides a measure of heteropolarity, and a correlation factor $-1 \leq f \leq 1$ that determines how the electronic motion is correlated. $f=1$ means that an electron is completely excluded from one domain if the other is already in it (positive correlation) and $f=-1$ implies that the two electrons are always found together within the same domain (negative correlation). The correlation factor here defined plays the same role as that used in density matrix theory, where $\rho^{2}\left(r_{1}, r_{2}\right)=\rho\left(r_{1}\right) \rho\left(r_{2}\right)(1-f)$. The $(p, f)$ pair describes fully a 2c,2e link at this level: $p(2,0)=p^{2}-p(1-p) f, p(1,1)=2 p(1-p)(1+f)$ and $p(0,2)=(1-p)^{2}-p(1-p) f .^{9}$

If we use these $p, f$ parameters, the covalent bond order becomes $\delta=4 p(1-p)(1-f)$. An ionic bond order $\iota=-Q_{a} Q_{b}$ where $Q$ is the net charge of a center has also been defined. ${ }^{10}$ 
In standard weakly correlated bonds with positive $f \sim 0$, the EDF is close to binomial, and $\delta$ peaks at $\delta=1$ for a purely covalent homopolar link with $p=1 / 2$. As electron correlation, $f$, or polarity, $p$, increases $\delta$ decreases. Moreover, for non-correlated links with $f=0 \iota=1-\delta$ so, in agreement with standard wisdom, the ionic and covalent bond orders are inversely correlated.

When $f$ deviates from zero, the model describes positively or negatively correlated bonds. The latter case implies a bosonization of the link. Electrons try to delocalize together, giving rise to very large fluctuations. The most extreme $2 \mathrm{c}, 2 \mathrm{e}$ case with $\delta=2$ occurs when $p(0,2)=$ $p(2,0)=1 / 2$ and $p(1,1)=0$, i.e. when there is a resonance between the two non-orthogonal valence bond (NOVB) ionic structures. Thus, $f<0$ serves to separate cleanly, in real space, large fluctuations from the standard bonding regime.

Several rigorous bond-energy bond-order $(\mathrm{BEBO})^{10}$ relations can be uncovered using these real space descriptors. Under the IQA perspective a multipolar expansions shows that the first order ionic and covalent energies are immediately related to their corresponding bond orders. For an interaction between atoms $A$ and $B$,

$$
E_{\text {ion }}^{A B} \sim-\frac{\iota^{A B}}{R_{i j}} \quad E_{\text {cov }}^{A B} \sim-\frac{1}{2} \frac{\delta^{A B}}{R_{A B}} .
$$

Notice that the arguments posed in Section 3 show that in the absence of ionic contributions (delocalization) a system is unbound. This means, for instance, that the good behavior of the conventional covalent Heitler-London function of $\mathrm{H}_{2}$ is due to the large overlap of the $\mathrm{H}$ 1s functions, that introduce a considerable amount of real space ionic terms.

\subsection{Local spin}

Spin densities have been used for almost three quarters of a century to understand the distribution of spin (and magnetism) in molecular systems. However, the spin density for a singlet state is identically zero everywhere, so that a question remains on how to quantify and localize spins in antiferromagnetic systems, or in singlet di- or polyradicals in molecules. This quest leads to the concept of local spin.

The expectation value of the $\left\langle\hat{S}^{2}\right\rangle$ operator for an arbitrary $N$-electron molecular system described by the wave function $\Psi$ can be expressed as

$$
\begin{aligned}
\left\langle\hat{S}^{2}\right\rangle & =\frac{3}{4} \int \rho\left(\boldsymbol{r}_{1}\right) d \boldsymbol{r}_{1} \\
& +\iint\left[-\frac{1}{4} \rho^{2}\left(\boldsymbol{r}_{1}, \boldsymbol{r}_{2} ; \boldsymbol{r}_{1}, \boldsymbol{r}_{2}\right)-\frac{1}{2} \rho^{2}\left(\boldsymbol{r}_{1}, \boldsymbol{r}_{2} ; \boldsymbol{r}_{2}, \boldsymbol{r}_{1}\right)\right] d \boldsymbol{r}_{1} d \boldsymbol{r}_{2},
\end{aligned}
$$

where $\rho\left(\boldsymbol{r}_{1}\right)$ is the electron density at point $\boldsymbol{r}_{1}$ and $\rho^{2}\left(\boldsymbol{r}_{1}, \boldsymbol{r}_{2} ; \boldsymbol{r}_{1}, \boldsymbol{r}_{2}\right)$ is the spinless diagonal second-order density, normalized to $N$ and $N(N-1)$, respectively. Considering the Dirac representation $\hat{S}^{2}=-N(N-4) / 4+\hat{O}$, where $\hat{O}=\sum_{i<j} \hat{p}_{i j}^{\sigma}$ and $\hat{p}_{i j}^{\sigma}$ is the operator interchanging the spin coordinates of electrons $i$ and $j$, the first two terms in eq 12 add to $-N(N-4) / 4$ and the second one is $\langle\Psi|\hat{O}| \Psi\rangle=-\frac{1}{2} \int \rho^{2}\left(\boldsymbol{r}_{1}, \boldsymbol{r}_{2} ; \boldsymbol{r}_{2}, \boldsymbol{r}_{1}\right) d \boldsymbol{r}_{1} d \boldsymbol{r}_{2}$.

The concept of local spin answers the question of how to distribute $\left\langle\hat{S}^{2}\right\rangle$ in atomic, $\left\langle\hat{S}^{2}\right\rangle_{A}$, and inter-atomic contributions, $\left\langle\hat{S}^{2}\right\rangle_{A B}$, in such a way that

$$
\left\langle\hat{S}^{2}\right\rangle=\sum_{A}\left\langle\hat{S}^{2}\right\rangle_{A}+\sum_{A \neq B}\left\langle\hat{S}^{2}\right\rangle_{A B},
$$

where $A$ and $B$ run over all the atoms of the system or, in general, over the different groups of atoms in which the molecule has been divided. This can generally be done by assigning atomic projectors, as done by Clark and Davidson. ${ }^{11}$ We thus define atomic regions, in our case using 
the QTAIM, and define the one-electron projector for electron $i$ as $P_{A}(i)=1$ if $i \in \Omega_{A}$ and $P_{A}(i)=0$ if $i \notin \Omega_{A}$, where $\Omega_{A}$ is the QTAIM atomic basin associated to atom $A$ (that we will simply call $A$ from now on). With this definition,

$$
\begin{aligned}
\left\langle\hat{S}_{A}^{2}\right\rangle & =\frac{3}{4} \int_{A} \rho\left(\boldsymbol{r}_{1}\right) d \boldsymbol{r}_{1}+\int_{A} \int_{A} F\left(\boldsymbol{r}_{1}, \boldsymbol{r}_{2}\right) d \boldsymbol{r}_{1} d \boldsymbol{r}_{2}, \\
\left\langle\hat{\boldsymbol{S}}_{A} \cdot \boldsymbol{S}_{B}\right\rangle & =\int_{A} \int_{B} F\left(\boldsymbol{r}_{1}, \boldsymbol{r}_{2}\right) d \boldsymbol{r}_{1} d \boldsymbol{r}_{2}, \quad \text { where } \\
F\left(\boldsymbol{r}_{1}, \boldsymbol{r}_{2}\right) & =-\frac{1}{4} \rho^{2}\left(\boldsymbol{r}_{1}, \boldsymbol{r}_{2} ; \boldsymbol{r}_{1}, \boldsymbol{r}_{2}\right)-\frac{1}{2} \rho^{2}\left(\boldsymbol{r}_{1}, \boldsymbol{r}_{2} ; \boldsymbol{r}_{2}, \boldsymbol{r}_{1}\right) .
\end{aligned}
$$

This provides a fully consistent partition of the squared spin operator into atomic and interatomic coupling terms. A fully isolated unpaired electron will offer a value for its local spin equal to $3 / 4$. As this delocalizes, the local spin will quench. In this sense, spin quenching and electron delocalization are fruitfully coupled together.

For instance, in the case of a single determinant closed-shell wavefunction (SDW),

$$
\begin{aligned}
& \rho_{2}\left(\boldsymbol{r}_{1}, \boldsymbol{r}_{2} ; \boldsymbol{r}_{1}, \boldsymbol{r}_{2}\right)=\rho\left(\boldsymbol{r}_{1} ; \boldsymbol{r}_{1}\right) \rho\left(\boldsymbol{r}_{2} ; \boldsymbol{r}_{2}\right)-\frac{1}{2} \rho\left(\boldsymbol{r}_{1} ; \boldsymbol{r}_{2}\right) \rho\left(\boldsymbol{r}_{2} ; \boldsymbol{r}_{1}\right), \quad \text { and } \\
& \rho_{2}\left(\boldsymbol{r}_{1}, \boldsymbol{r}_{2} ; \boldsymbol{r}_{2}, \boldsymbol{r}_{1}\right)=\rho\left(\boldsymbol{r}_{1} ; \boldsymbol{r}_{2}\right) \rho\left(\boldsymbol{r}_{2} ; \boldsymbol{r}_{1}\right)-\frac{1}{2} \rho\left(\boldsymbol{r}_{1} ; \boldsymbol{r}_{1}\right) \rho\left(\boldsymbol{r}_{2} ; \boldsymbol{r}_{2}\right),
\end{aligned}
$$

so that

$$
-\frac{1}{4} \rho_{2}\left(\boldsymbol{r}_{1}, \boldsymbol{r}_{2} ; \boldsymbol{r}_{1}, \boldsymbol{r}_{2}\right)-\frac{1}{2} \rho_{2}\left(\boldsymbol{r}_{1}, \boldsymbol{r}_{2} ; \boldsymbol{r}_{2}, \boldsymbol{r}_{1}\right)=-\frac{3}{8} \rho\left(\boldsymbol{r}_{1}, \boldsymbol{r}_{2}\right) \rho\left(\boldsymbol{r}_{2}, \boldsymbol{r}_{1}\right)
$$

Then,

$$
\left\langle S^{2}\right\rangle=\frac{3}{4} \int \rho(\boldsymbol{r}) d \boldsymbol{r}-\frac{3}{8} \iint \rho\left(\boldsymbol{r}_{1}, \boldsymbol{r}_{2}\right) \rho\left(\boldsymbol{r}_{2}, \boldsymbol{r}_{1}\right) d \boldsymbol{r}_{1} \boldsymbol{r}_{2}
$$

The localization index inside $A$ is given by

$$
\lambda^{A}=\int_{A} \int_{A} \rho_{x c}\left(\boldsymbol{r}_{1}, \boldsymbol{r}_{2}\right) d \boldsymbol{r}_{1} d \boldsymbol{r}_{2}
$$

where $\rho_{x c}$ is the exchange-correlation density. Since, $\rho_{x c}\left(\boldsymbol{r}_{1} ; \boldsymbol{r}_{2}\right)=\frac{1}{2} \rho\left(\boldsymbol{r}_{1} ; \boldsymbol{r}_{2}\right) \rho\left(\boldsymbol{r}_{2} ; \boldsymbol{r}_{1}\right)$ in the case of a closed-shell SDW, eq 14 becomes

$$
\left\langle S_{A}^{2}\right\rangle=\frac{3}{4}\left(N_{A}-\lambda_{A}\right)
$$

where $N_{A}=\int \rho(\boldsymbol{r}) d \boldsymbol{r}$ is the average number on electron in $A$. Similarly, the delocalization index between $A$ and $B\left(\delta^{A B}\right)$, a measure of the bond order between both atoms, is given by

$$
\delta^{A B}=2 \int_{A} \int_{B} \rho_{x c}\left(\boldsymbol{r}_{1}, \boldsymbol{r}_{2}\right) d \boldsymbol{r}_{1} d \boldsymbol{r}_{2},
$$

and, from eqs 15,16 , and 19 , its value for a closed-shell SDW is related to $\left\langle S_{A B}^{2}\right\rangle$ by

$$
\left\langle S_{A B}^{2}\right\rangle=-\frac{3}{8} \delta^{A B} .
$$

In the case of a $2 \mathrm{c}, 2 \mathrm{e}$ pure single bond with $\delta=1$, this means that the local spin of each center will be quenched from $3 / 4$ to $3 / 8$, and that the spin coupling will change from $-3 / 4$ to $-3 / 8$. We have shown that there is an intimate relationship between local spins and electron distributions. ${ }^{12}$ For instance in the symmetric $2 \mathrm{c}, 2 \mathrm{e}$ case with $p(2,0)=p(0,2)=1 / 4$, the local spin of each center will be due to the resonance structure where each electron is located in a center, since when the two ionic configurations will lead to null $\left\langle S_{A}^{2}\right\rangle$. Since $p(1,1)=1 / 2$ the local spin is $1 / 2 \times 3 / 4=3 / 8$. This EDF perspective is extremely fruitful. For two localized isolated spins coupled to a triplet, $\left\langle S_{A}^{2}\right\rangle=3 / 4$ and $\left\langle S_{A B}^{2}\right\rangle=1 / 4$. 


\section{The Coulson-Fischer state and its real space analysis}

Let us consider a system with two equivalent centers $(a, b)$ and two magnetic electrons. Two localized functions $\phi_{a}, \phi_{b}$ (or just $a, b$ ) with overlap $\langle a \mid b\rangle=S$ are used. As stated in the manuscript, two ${ }^{1} \Sigma_{g}^{+}$singlets and one ${ }^{3} \Sigma_{u}^{+}$triplet come out from this basis. In the HeitlerLondon or Valence Bond (VB) framework, they are

$$
\begin{aligned}
\Psi_{S, \text { cov }} & =\frac{1}{\sqrt{2\left(1+S^{2}\right)}}(a(1) b(2)+b(1) a(2)) \frac{1}{\sqrt{2}}(\uparrow \downarrow-\downarrow \uparrow), \\
\Psi_{S, \text { ion }} & =\frac{1}{\sqrt{2\left(1+S^{2}\right)}}(a(1) a(2)+b(1) b(2)) \frac{1}{\sqrt{2}}(\uparrow \downarrow-\downarrow \uparrow) . \\
\Psi_{T} & =\frac{1}{\sqrt{2\left(1-S^{2}\right)}}(a(1) b(2)-b(1) a(2)) \frac{1}{\sqrt{2}}(\uparrow \downarrow+\downarrow \uparrow) .
\end{aligned}
$$

Similarly, the MO basis is formed from the gerade and ungerade combinations, $\phi_{g}, \phi_{u}$ (or simply $g, u)$

$$
g=\frac{1}{\sqrt{2(1+S)}}(a+b), u=\frac{1}{\sqrt{2(1-S)}}(a-b),
$$

so that two independent ${ }^{1} \Sigma_{g}^{+}$singlets are:

$$
\Psi_{g g}=\Psi_{\mathrm{HF}}=|g \bar{g}|, \Psi_{u u}=|u \bar{u}| .
$$

while the ${ }^{3} \Sigma_{u}^{+}$triplet $\left(M_{S}=0\right.$ component $)$ is given by

$$
\Psi_{T}=\frac{1}{\sqrt{2}}(|g \bar{u}|-|u \bar{g}|) .
$$

The variational space of the singlets is two dimensional, so that the lowest energy singlet can be written as

$$
\Psi_{S}=c \Psi_{c o v}+i \Psi_{i o n}=\lambda \Psi_{g g}+\mu \Psi_{u u} .
$$

The $\lambda^{2}+\mu^{2}=1$ condition allows to map easily the full spectrum of the singlets by using a $\omega$-angle polar representation such that $\lambda=\cos (\omega / 2), \mu=-\sin (\omega / 2), \omega \in[-\pi, \pi]$.

Localized orthogonal Coulson-Fischer orbitals can be obtained from the localized $a, b$ functions as $\varphi_{a, b}=(g \pm u) / \sqrt{2}$. With them, the Heitler-London-like Coulson-Fischer singlet state is obtained as the covalent function in Eq. 25. In terms of the $g, u$ functions,

$$
\Psi_{S}^{o}=\frac{1}{\sqrt{2}}(|g \bar{g}|-|u \bar{u}|) .
$$

The triplet built with this localized orthogonal functions (OLOs) is equal to the the canonical one.

Working in the orthogonal $\lambda, \mu$ representation provides direct easy access to all reduced density matrices. For the singlets, the $|g \bar{g}|$ and $|u \bar{u}|$ determinants differ in two spinorbitals, so only the 2RDM has coupling terms,

$$
\begin{aligned}
\rho\left(\boldsymbol{r} ; \boldsymbol{r}^{\prime}\right) & =2 \lambda^{2} g(\boldsymbol{r}) g\left(\boldsymbol{r}^{\prime}\right)+2 \mu^{2} u(\boldsymbol{r}) u\left(\boldsymbol{r}^{\prime}\right) \\
\rho_{2}\left(\boldsymbol{r}_{1}, \boldsymbol{r}_{2}\right) & =2 \lambda^{2} g^{2}\left(\boldsymbol{r}_{1}\right) g^{2}\left(\boldsymbol{r}_{2}\right)+2 \mu^{2} u^{2}\left(\boldsymbol{r}_{1}\right) u^{2}\left(\boldsymbol{r}_{2}\right)+4 \lambda \mu g u\left(\boldsymbol{r}_{1}\right) g u\left(\boldsymbol{r}_{2}\right) .
\end{aligned}
$$

Similarly, for the triplet

$$
\begin{aligned}
\rho\left(\boldsymbol{r} ; \boldsymbol{r}^{\prime}\right) & =g(\boldsymbol{r}) g\left(\boldsymbol{r}^{\prime}\right)+u(\boldsymbol{r}) u\left(\boldsymbol{r}^{\prime}\right) \\
\rho_{2}\left(\boldsymbol{r}_{1}, \boldsymbol{r}_{2}\right) & =g^{2}\left(\boldsymbol{r}_{1}\right) u^{2}\left(\boldsymbol{r}_{2}\right)+u^{2}\left(\boldsymbol{r}_{1}\right) g^{2}\left(\boldsymbol{r}_{2}\right)-2 g u\left(\boldsymbol{r}_{1}\right) g u\left(\boldsymbol{r}_{2}\right) .
\end{aligned}
$$


Since in $\Psi_{S}^{o} \lambda=-\mu=1 / \sqrt{2}$, the first order density matrix of the Coulson-Fischer singlet and triplet are equal, and the pair densities differ in

$$
\Delta \rho_{2}\left(\boldsymbol{r}_{1}, \boldsymbol{r}_{2}\right)_{S, T}=\left(g^{2}\left(\boldsymbol{r}_{1}\right)-u^{2}\left(\boldsymbol{r}_{1}\right)\left(g^{2}\left(\boldsymbol{r}_{2}\right)-u^{2}\left(\boldsymbol{r}_{2}\right),\right.\right.
$$

which can be written as $2 \varphi_{a}\left(\boldsymbol{r}_{1}\right) \varphi_{b}\left(\boldsymbol{r}_{1}\right) \varphi_{a}\left(\boldsymbol{r}_{2}\right) \varphi_{b}\left(\boldsymbol{r}_{2}\right)$. Notice that this difference integrates to zero.

For a two electron system the probability of finding the two electrons in a given atomic basin is given by

$$
p(2,0)=\frac{1}{2} \int_{\Omega} d \boldsymbol{r}_{1} \int_{\Omega} d \boldsymbol{r}_{2} \rho_{2}\left(\boldsymbol{r}_{1}, \boldsymbol{r}_{2}\right),
$$

and given that the orthogonality of $\varphi_{a}$ and $\varphi_{b}$ also implies that $\int_{\Omega} \varphi_{a}(\boldsymbol{r}) \varphi_{b}(\boldsymbol{r})=0$, then $p(2,0)$ is equal for the Coulson-Fischer singlet and triplet, and so are $p(1,1)$ and the variances and covariances (localization and delocalization indices).

The Coulson-Fischer singlet and triplet states can thus not be distinguished by any oneelectron property or even by the atomic condensed electron distribution functions. Only when $\Delta \rho_{2}$ is weighted with the inverse interelectron distance to form the $\Delta V_{e e}$ operator we obtain an overall energy difference. This points clearly to a different average separation of electrons in both states, as shown in Fig. 1 of the ms.

\section{Methodological details}

All calculations in the $\mathrm{H}-\mathrm{X}-\mathrm{H}^{n+}$ systems with $\mathrm{X}=\mathrm{He}, \mathrm{Be}$ and $n=0,2$ have been performed with the STO-6G minimal and the extended 6-311G(p) Pople basis sets at the Hartree-Fock (HF) and Full Configuration Interaction (FCI) levels with the GAMESS code. ${ }^{13}$ Calculations on the Li-Be-Li system were also performed with GAMESS and the $6-311+\mathrm{G}^{* *}$ basis set.

In $\mathrm{H}-\mathrm{He}-\mathrm{H}$ we also substituted the He atom by an effective core potential, written with the following algebraic structure ${ }^{14}$ :

$$
\begin{gathered}
V_{\text {eff }}=V_{L}(r)+\sum_{\lambda=0}^{L-1} \sum_{\mu=-\lambda}^{\lambda}\left|Y_{\lambda \mu}\right\rangle V_{\lambda-L}(r)\left\langle Y_{\lambda \mu}\right|, \\
V_{\lambda-L}(r)=V_{\lambda}(r)-V_{L}(r) .
\end{gathered}
$$

Here, $L-1$ is the maximum angular momentum of the excluded core electrons, and the $l=\lambda-L$ dependent $V$ 's impose orthogonality constraints that avoid the collapse of the valence orbitals onto the bridge (core) states. The spherical harmonics and radial functions are centered at the He nucleus, and the $V_{l}(r)$ potentials are expressed as linear combinations of gaussians:

$$
V_{l}(r)=\sum_{i=1}^{N} B_{i}^{l} r^{n_{i}^{l}} \exp \left(-\alpha_{i}^{l} r^{2}\right)
$$

Here we have decided to use $L=1$, so the ECP is a sum of a $\mathrm{P}$ potential and a $\mathrm{S}-\mathrm{P}$ one. The $B_{i}, n_{i}$, and $\alpha_{i}$ coefficients are given in the following table: 


\begin{tabular}{rrrr}
\hline$n_{i}$ & $\mathrm{~B}_{i}$ & $n_{i}$ & $\alpha_{i}$ \\
\hline $\mathrm{P}$ & & & \\
\hline 1 & -0.11866700 & 2 & 0.80780000 \\
2 & -1.21779400 & 2 & 2.55000001 \\
3 & -1.37580501 & 1 & 7.25349998 \\
\hline $\mathrm{S}-\mathrm{P}$ & & & \\
\hline 1 & 24.33369899 & 2 & 0.79980000 \\
2 & -20.66639709 & 2 & 0.77410000 \\
3 & -1.14289200 & 1 & 1.19430000 \\
4 & 2.99401900 & 0 & 2.19990000 \\
\hline
\end{tabular}

All real space analyses have been performed with our QTAIM in-house codes. IQA decompositions with PROMOLDEN, EDF probabilities with EDF, and local spin partitions with NRDM. They are freely available from the authors upon request, by writing to either ampendas@uniovi.es or evelio@uniovi.es. IQA integrations were performed using $\beta$-spheres with a radius $90 \%$ as large as the smallest atomic QTAIM bonded radius of each atom, with 434 and 5810 angular Lebedev points inside and outside the sphere, respectively, maximum $l$ expansions up to $l=10,4$ outside and inside, respectively, and 700 and 400 trapezoidal and Gauss-Chebychev radial points in the same order.

\section{Raw Results}

We offer here a list of results for the systems explored. We show the FCI 6-311G(p) data for $\mathrm{H}-\mathrm{He}-\mathrm{H}$. Atomic numbering is always 1 for $\mathrm{He}$ and 2,3 for $\mathrm{H}$ atoms except for EDFs, in which the central He atom is atom 2. All data in atomic units. Geometries, canonical orbital energies and natural orbital occupations, main coefficients $(>0.01)$ of the FCI determinant expansion in the canonical basis with positive/negative labels tagging alpha and beta spin projections, IQA descriptors, EDF and local spin decompositions are found in consecutive order.

\subsection{H-He-H LS}

\begin{tabular}{lcccc} 
ATOM & ATOMIC & \multicolumn{3}{c}{ COORDINATES (BOHR) } \\
& CHARGE & $\mathrm{X}$ & $\mathrm{Y}$ & $\mathrm{Z}$ \\
HE & 2.0 & 0.0000000000 & 0.0000000000 & 0.0000000000 \\
H & 1.0 & 0.0000000000 & 0.0000000000 & -2.3786536587 \\
H & 1.0 & 0.0000000000 & 0.0000000000 & 2.3786536587
\end{tabular}

\section{EIGENVECTORS}

$\begin{array}{ccccc}1 & 2 & 3 & 4 & 5 \\ -1.0556 & -0.3124 & -0.0269 & 0.3836 & 0.3928 \\ \text { AG } & \text { B1U } & \text { AG } & \text { AG } & \text { B } 1 \text { U }\end{array}$

NATURAL ORBITALS IN ATOMIC ORBITAL BASIS

$\begin{array}{ccccc}1 & 2 & 3 & 4 & 5 \\ 1.9818 & 1.4942 & 0.5044 & 0.0066 & 0.0039 \\ \mathrm{AG} & \mathrm{B} 1 \mathrm{U} & \mathrm{AG} & \mathrm{AG} & \mathrm{B} 1 \mathrm{U}\end{array}$


NUCLEUS-ELECTRON POTENTIAL ENERGY = NUCLEUS-NUCLEUS POTENTIAL ENERGY $=$

TOTAL POTENTIAL ENERGY = TOTAL KINETIC ENERGY = $\operatorname{VIRIAL} \operatorname{RATIO}(\mathrm{V} / \mathrm{T})=$
$-12.3468267482$

1.8918264891

$-7.8027238875$

3.9699518760

1.9654454591

COEFFICIENT/ OCCUPIED ACTIVE SPIN ORBITALS

$\begin{array}{rllll}0.8600731486 \mathrm{E}+00 & 1 & 2 & -1 & -2 \\ -0.4983389596 \mathrm{E}+00 & 1 & 3 & -1 & -3 \\ -0.3999934103 \mathrm{E}-01 & 2 & 4 & -2 & -4 \\ -0.2852488019 \mathrm{E}-01 & 2 & 6 & -2 & -6 \\ -0.2852488019 \mathrm{E}-01 & 2 & 7 & -2 & -7 \\ -0.2779527407 \mathrm{E}-01 & 2 & 3 & -2 & -4 \\ -0.2779527407 \mathrm{E}-01 & 2 & 4 & -2 & -3 \\ 0.2267670752 \mathrm{E}-01 & 3 & 4 & -3 & -4 \\ -0.2105905507 \mathrm{E}-01 & 2 & 5 & -2 & -5 \\ -0.1999847491 \mathrm{E}-01 & 2 & 3 & -2 & -3 \\ -0.1681461010 \mathrm{E}-01 & 1 & 5 & -1 & -5 \\ 0.1674848128 \mathrm{E}-01 & 3 & 7 & -3 & -7 \\ 0.1674848128 \mathrm{E}-01 & 3 & 6 & -3 & -6 \\ 0.1669365677 \mathrm{E}-01 & 1 & 8 & -2 & -3 \\ 0.1669365677 \mathrm{E}-01 & 2 & 3 & -1 & -8 \\ -0.1549172969 \mathrm{E}-01 & 1 & 2 & -3 & -5 \\ -0.1549172969 \mathrm{E}-01 & 3 & 5 & -1 & -2 \\ 0.1425802262 \mathrm{E}-01 & 3 & 5 & -3 & -5 \\ 0.1239230589 \mathrm{E}-01 & 1 & 3 & -2 & -8 \\ 0.1239230589 \mathrm{E}-01 & 2 & 8 & -1 & -3 \\ 0.1122241557 \mathrm{E}-01 & 1 & 9 & -2 & -5 \\ 0.1122241557 \mathrm{E}-01 & 2 & 5 & -1 & -9 \\ -0.1070696621 \mathrm{E}-01 & 1 & 9 & -1 & -9 \\ -0.1031310282 \mathrm{E}-01 & 1 & 8 & -1 & -8\end{array}$

Atomic Contributions for neq: 1

Atom number

: 1

\begin{tabular}{|c|c|c|c|c|c|c|}
\hline kinetic energy & $=$ & 2.90411770 & & & & \\
\hline potential energy & $=$ & -5.73496917 & & & & \\
\hline electron repulsion & $=$ & 1.05361204 & & & & \\
\hline ---coulomb & $=$ & 2.0 & 7270320 & & & \\
\hline$---e x c h+\operatorname{corr}$ & $=$ & -1.0 & 1909116 & & & \\
\hline$---\operatorname{self}$ & $=$ & 0.0 & 0000000 & & & \\
\hline el-own-nuc attraction & $=$ & -6.72888677 & & & & \\
\hline net energy & $=$ & -2.77115703 & & & & \\
\hline interaction energy & $=$ & -0.11938889 & & & & \\
\hline additive energy & $=$ & -2.83085148 & & & & \\
\hline effective energy & $=$ & -2.89054592 & & & & \\
\hline $2 \mathrm{~T}+\mathrm{V}$ & $=$ & 0.07326622 & & & & \\
\hline Int rho_2 & $=$ & 2.28136968 & & & & \\
\hline Integ rho_2 $\mathrm{J}$ & $=$ & 4.04937901 & & & & \\
\hline Integ rho_2 XC & $=$ & 1.76800933 & & & & \\
\hline SUM-RULE-TEST & $=$ & 2.01224996 & AND SHOULD BE & 2.01230689 & & \\
\hline$===================$ In & teract & ion with atom: & $2===========$ & $============8$ & $=============$ & $=======$ \\
\hline (NN, EN, NE, EE, Inter) & & 0.84081177 & -0.83720355 & -0.72925369 & 0.66595102 & -0.05969444 \\
\hline EE wself : (coul, XC, se] & lf) & 0.72837773 & -0.06242671 & 0.00000000 & & \\
\hline EE woself: (coul, XC) & & 0.72837773 & -0.06242671 & & & \\
\hline Coul comp.: (longr, shor & rtr) & $-0.342826 \mathrm{E}+03$ & $0.343554 \mathrm{E}+03$ & & & \\
\hline Classical Int. (Long, Tot & tal) & $-0.343673 E+03$ & $0.273227 \mathrm{E}-02$ & & & \\
\hline RHO_2 Integ comp (TOT, J, & , XC) & 1.87763990 & 1.99976022 & 0.12212032 & & \\
\hline
\end{tabular}




\begin{tabular}{|c|c|c|c|c|c|c|}
\hline \multirow{2}{*}{$\begin{array}{l}================== \\
(N N, E N, N E, E E, \text { Inter })\end{array}$} & \multicolumn{2}{|c|}{ Interaction with atom: } & \multicolumn{4}{|c|}{ 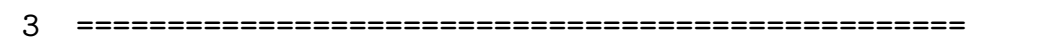 } \\
\hline & & 0.84081177 & -0.83720355 & -0.72925369 & 0.66595102 & -0.05969444 \\
\hline \multicolumn{2}{|c|}{ EE wself : (coul, XC,self) } & 0.72837773 & -0.06242671 & 0.00000000 & & \\
\hline \multicolumn{2}{|l|}{ EE woself: (coul, XC) } & 0.72837773 & -0.06242671 & & & \\
\hline \multicolumn{2}{|c|}{ Coul comp.: (longr, shortr) } & $-0.342826 \mathrm{E}+03$ & $0.343554 \mathrm{E}+03$ & & & \\
\hline \multicolumn{2}{|c|}{ Classical Int. (Long,Total) } & $-0.343673 E+03$ & $0.273227 \mathrm{E}-02$ & & & \\
\hline \multicolumn{2}{|c|}{ RHO_2 Integ comp (TOT, J, XC) } & 1.87763990 & 1.99976022 & 0.12212032 & & \\
\hline \multicolumn{2}{|l|}{$F_{-} A B(X C)$} & 0.24424063 & & & & \\
\hline \multirow{2}{*}{\multicolumn{2}{|c|}{$\begin{array}{l}\text { Atomic Contributions for neq: } \\
\text { Atom number }\end{array}$}} & : 2 & & & & \\
\hline & & : 2 & & & & \\
\hline \multicolumn{7}{|c|}{ kinetic energy $\quad=\quad 0.53283705$} \\
\hline potential energy & $=$ & -1.03382920 & & & & \\
\hline electron repulsion & $=$ & 0.04462788 & & & & \\
\hline$---c o u l o m b$ & $=$ & 0.3 & 3016294 & & & \\
\hline$---e x c h+\operatorname{corr}$ & $=$ & -0.2 & 8553507 & & & \\
\hline$---\operatorname{self}$ & $=$ & 0.0 & 0000000 & & & \\
\hline el-own-nuc attraction & $=$ & -1.04598508 & & & & \\
\hline net energy & $=$ & -0.46852015 & & & & \\
\hline interaction energy & $=$ & -0.06494400 & & & & \\
\hline additive energy & $=$ & -0.50099215 & & & & \\
\hline effective energy & $=$ & -0.53346415 & & & & \\
\hline $2 \mathrm{~T}+\mathrm{V}$ & $=$ & 0.03184490 & & & & \\
\hline Int rho_2 & $=$ & 0.15718711 & & & & \\
\hline Integ rho_2 J & $=$ & 0.98756894 & & & & \\
\hline \multirow{2}{*}{$\begin{array}{l}\text { Integ rho_2 XC (F_AA) } \\
\text { SUM-RULE-TEST }\end{array}$} & $=$ & 0.83038183 & & & & \\
\hline & $=$ & 0.99371196 & AND SHOULD BE & 0.99376503 & & \\
\hline \multicolumn{3}{|c|}{$==================$ Interaction with atom: } & $3==========$ & $============$ & $==========$ & $======$ \\
\hline \multicolumn{2}{|l|}{ (NN , EN , NE, EE, Inter) } & 0.21020294 & -0.19640851 & -0.19640851 & 0.17736451 & -0.00524956 \\
\hline \multicolumn{2}{|c|}{ EE wself : (coul, XC, self) } & 0.18442648 & -0.00706197 & 0.00000000 & & \\
\hline EE woself: (coul, XC) & & 0.18442648 & -0.00706197 & & & \\
\hline Coul comp.: (longr, sho & rtr) & $0.185393 \mathrm{E}+00$ & $-0.966655 \mathrm{E}-03$ & & & \\
\hline Classical Int. (Long,To & tal) & $0.273221 \mathrm{E}-02$ & $0.181241 \mathrm{E}-02$ & & & \\
\hline RHO_2 Integ comp (TOT, J & , XC) & 0.94635913 & 0.98756894 & 0.04120981 & & \\
\hline$F_{-} A B \quad(X C)$ & & 0.08241962 & & & & \\
\hline
\end{tabular}

\# M-BASINS SPINLESS ELECTRON DISTRIBUTION FUNCTION

\begin{tabular}{|c|c|c|c|c|}
\hline \multicolumn{4}{|c|}{ \# NUMBER OF GROUPS } & \\
\hline \multicolumn{3}{|c|}{ \# TOTAL NUMBER OF PROBABILITIES } & & 15 \\
\hline $\begin{array}{l}\#^{-}- \\
\#\end{array}$ & Probability & $\mathrm{n} 1(\mathrm{H})$ & $\mathrm{n} 2(\mathrm{He})$ & n3 (H) \\
\hline \# & 0.7278283855326309 & 1 & 2 & 1 \\
\hline \# & 0.0570771504525981 & 1 & 3 & 0 \\
\hline$\#$ & 0.0570771504525981 & 0 & 3 & 1 \\
\hline$\#$ & 0.0528440664176910 & 2 & 1 & 1 \\
\hline$\#$ & 0.0528440664176910 & 1 & 1 & 2 \\
\hline \# & 0.0228116412268904 & 2 & 2 & 0 \\
\hline \# & 0.0228116412268904 & 0 & 2 & 2 \\
\hline \# & 0.0041089204834032 & 0 & 4 & 0 \\
\hline \# & 0.0014400237727642 & 2 & 0 & 2 \\
\hline \# & 0.0002728138540450 & 3 & 1 & 0 \\
\hline$\#$ & 0.0002728138540450 & 0 & 1 & 3 \\
\hline$\#$ & 0.0002237579192957 & 1 & 0 & 3 \\
\hline$\#$ & 0.0002237579192957 & 3 & 0 & 1 \\
\hline$\#$ & 0.0000003780052079 & 0 & 0 & 4 \\
\hline$\#$ & 0.0000003780052079 & 4 & 0 & 0 \\
\hline
\end{tabular}


Average populations and localization indices

0.9936560505

\# $<\mathrm{n}(2)>$

$=0.9936560505$

$\#<n(3)>$

$\#<\mathrm{n}(2) \mathrm{n}(1)>$

2. 0120356812

$\#<\mathrm{n}(3) \mathrm{n}(1)>$

0.9936560505

\#<n( 3) n ( 2)

$=\quad 1.8774854281$

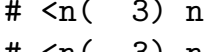

$=0.9463072938$

\# $<\mathrm{n}(3) \mathrm{n}($

2) $>$

1.8774854281

\# delta_( 111$)$

\# delta_( 2 2)

$\#$ delta_( 33$)$

1) $>=$

1. 6670330367

0.8301769172

$=\quad 1.7671513951 \%$ Localization $=87.8290$

$\%$ Localization $=83.5477$

$=0.8301769172 \%$ Localization $=83.5477$

\begin{tabular}{|c|c|c|c|c|}
\hline \multicolumn{5}{|c|}{ Delocalization indices } \\
\hline \# delta_( & 2 & 1) & $=$ & 0.2442239808 \\
\hline \# delta_( & 3 & 1) & $=$ & 0.082412090 \\
\hline$\#$ delta_( & 3 & 2) & $=$ & 0.244223980 \\
\hline \# delta_( & 3 & $21)$ & $=$ & 0.0107594207 \\
\hline
\end{tabular}

\# Fragment A formed by atoms 1: He

\# Fragment B formed by atoms 2 3: H, H

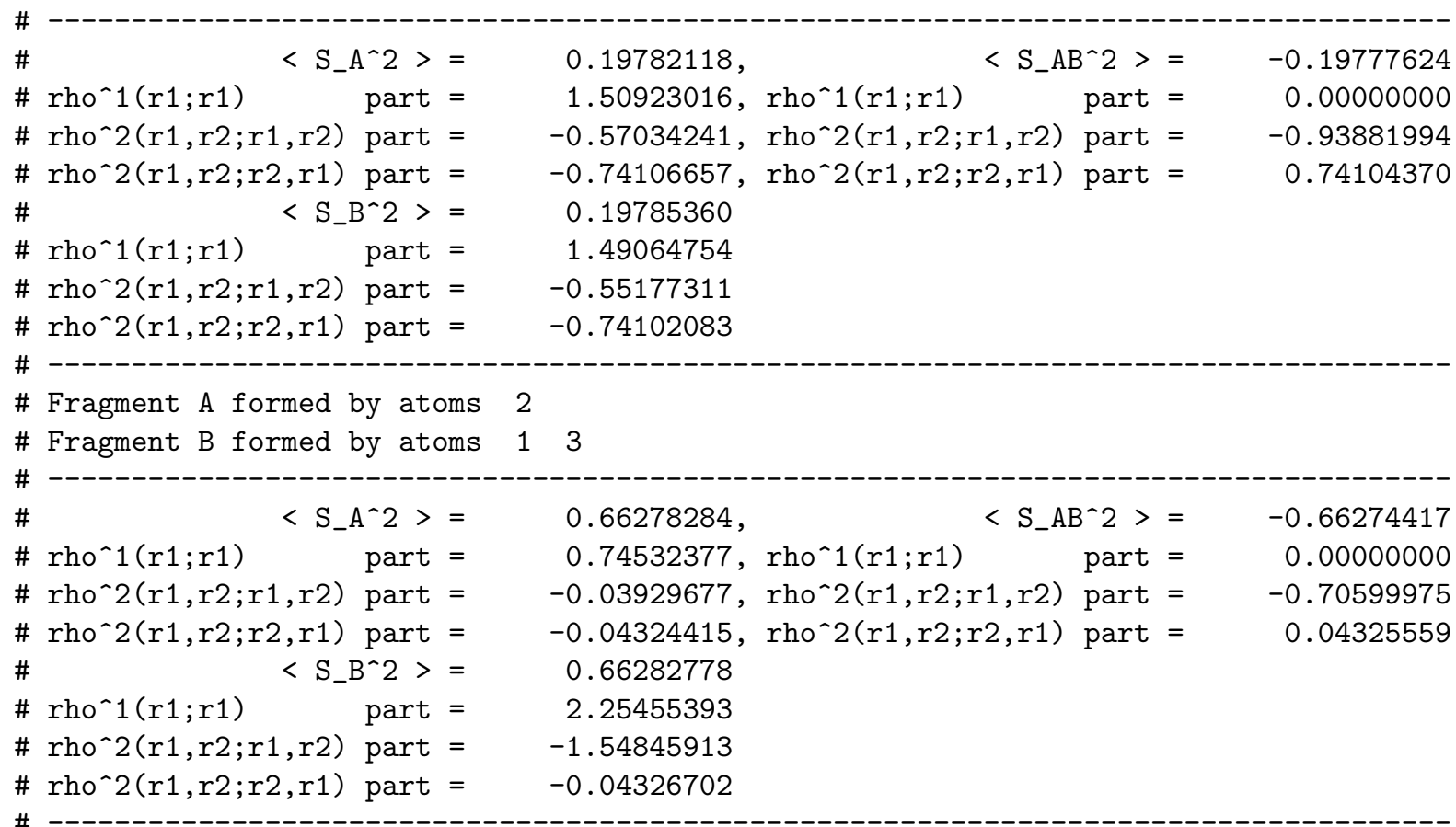

\subsection{H-He-H LT}

\begin{tabular}{lcccc} 
ATOM & ATOMIC & \multicolumn{3}{c}{ COORDINATES (BOHR) } \\
& CHARGE & $\mathrm{X}$ & $\mathrm{Y}$ & $\mathrm{Z}$ \\
$\mathrm{HE}$ & 2.0 & 0.0000000000 & 0.0000000000 & 0.0000000000 \\
$\mathrm{H}$ & 1.0 & 0.0000000000 & 0.0000000000 & -2.3786536587 \\
$\mathrm{H}$ & 1.0 & 0.0000000000 & 0.0000000000 & 2.3786536587
\end{tabular}

EIGENVECTORS 


$\begin{array}{ccccc}-0.9513 & -0.1854 & -0.0994 & 0.2801 & 0.3266 \\ \text { AG } & \text { B1U } & \text { AG } & \text { AG } & \text { B1U }\end{array}$

NATURAL ORBITALS IN ATOMIC ORBITAL BASIS

$\begin{array}{ccccc}1 & 2 & 3 & 4 & 5 \\ 1.9832 & 0.9993 & 0.9992 & 0.0067 & 0.0029 \\ A G & A G & B 1 U & A G & B 1 U\end{array}$

TOTAL ENERGY $=\quad-3.8116308380$

ELECTRON-ELECTRON POTENTIAL ENERGY =

2.6368800561

NUCLEUS-ELECTRON POTENTIAL ENERGY $=-12.4305485252$

NUCLEUS-NUCLEUS POTENTIAL ENERGY =

1.8918264891

TOTAL POTENTIAL ENERGY =

$-7.9018419800$

TOTAL KINETIC ENERGY =

4.0902111420

VIRIAL RATIO $(\mathrm{V} / \mathrm{T})=$

1.9318909723

COEFFICIENT/ OCCUPIED ACTIVE SPIN ORBITALS

$\begin{array}{rllll}0.7033287160 \mathrm{E}+00 & 1 & 2 & -1 & -3 \\ -0.7033287160 \mathrm{E}+00 & 1 & 3 & -1 & -2 \\ -0.3242931340 \mathrm{E}-01 & 2 & 4 & -3 & -4 \\ 0.3242931340 \mathrm{E}-01 & 3 & 4 & -2 & -4 \\ -0.2360802682 \mathrm{E}-01 & 2 & 6 & -3 & -6 \\ 0.2360802682 \mathrm{E}-01 & 3 & 6 & -2 & -6 \\ -0.2360802682 \mathrm{E}-01 & 2 & 7 & -3 & -7 \\ 0.2360802682 \mathrm{E}-01 & 3 & 7 & -2 & -7 \\ 0.2054077032 \mathrm{E}-01 & 2 & 3 & -2 & -4 \\ -0.2054077032 \mathrm{E}-01 & 2 & 4 & -2 & -3 \\ -0.2039663467 \mathrm{E}-01 & 2 & 5 & -3 & -5 \\ 0.2039663467 \mathrm{E}-01 & 3 & 5 & -2 & -5 \\ 0.2025185335 \mathrm{E}-01 & 1 & 8 & -2 & -3 \\ -0.2025185335 \mathrm{E}-01 & 2 & 3 & -1 & -8 \\ 0.1425105024 \mathrm{E}-01 & 1 & 4 & -3 & -4 \\ -0.1425105024 \mathrm{E}-01 & 3 & 4 & -1 & -4 \\ 0.1273827264 \mathrm{E}-01 & 1 & 4 & -2 & -3 \\ -0.1273827264 \mathrm{E}-01 & 2 & 3 & -1 & -4 \\ 0.1116495271 \mathrm{E}-01 & 1 & 3 & -3 & -9 \\ -0.1116495271 \mathrm{E}-01 & 3 & 9 & -1 & -3 \\ 0.1015371523 \mathrm{E}-01 & 2 & 3 & -3 & -5 \\ -0.1015371523 \mathrm{E}-01 & 3 & 5 & -2 & -3\end{array}$

Atomic Contributions for neq: 1

Atom number

: 1

$\begin{array}{llr}\text { kinetic energy } & = & 3.01022302 \\ \text { potential energy } & = & -5.84233420 \\ \text { electron repulsion } & = & 1.07085502 \\ \text {---coulomb } & = & 2 \\ \text {---exch+corr } & = & -1 \\ \text {---self } & = & 0 \\ \text { el-own-nuc attraction } & = & -6.86426410 \\ \text { net energy } & = & -2.78318607 \\ \text { interaction energy } & = & -0.09785023 \\ \text { additive energy } & = & -2.83211118 \\ \text { effective energy } & = & -2.88103630 \\ 2 \mathrm{~T}+\mathrm{V} & = & 0.17811183\end{array}$


Int rho_2

Integ rho_2 J

Integ rho_2 XC

SUM-RULE-TEST

$\begin{array}{lr}================= & \text { Interaction with atom: } \\ \text { (NN, EN, NE, EE, Inter) } & 0.84081177\end{array}$

EE wself : (coul, XC, self)

EE woself: (coul, XC)

0.71766594

0.71766594

Coul comp.: (longr, shortr) -0.888970E+04

Classical Int. (Long, Total) $-0.889066 \mathrm{E}+04$

RHO_2 Integ comp (TOT, J,XC)

F_AB (XC)
1.89495366

0.20957064
AND SHOULD BE 2.02181507

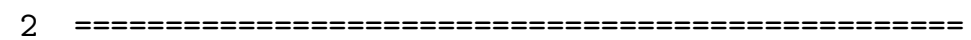
$\begin{array}{llll}-0.83941034 & -0.71619004 & 0.66586349 & -0.04892512\end{array}$ $-0.05180245 \quad 0.00000000$

$-0.05180245$

$0.889042 \mathrm{E}+04$

$0.287734 \mathrm{E}-02$

$1.99973898 \quad 0.10478532$

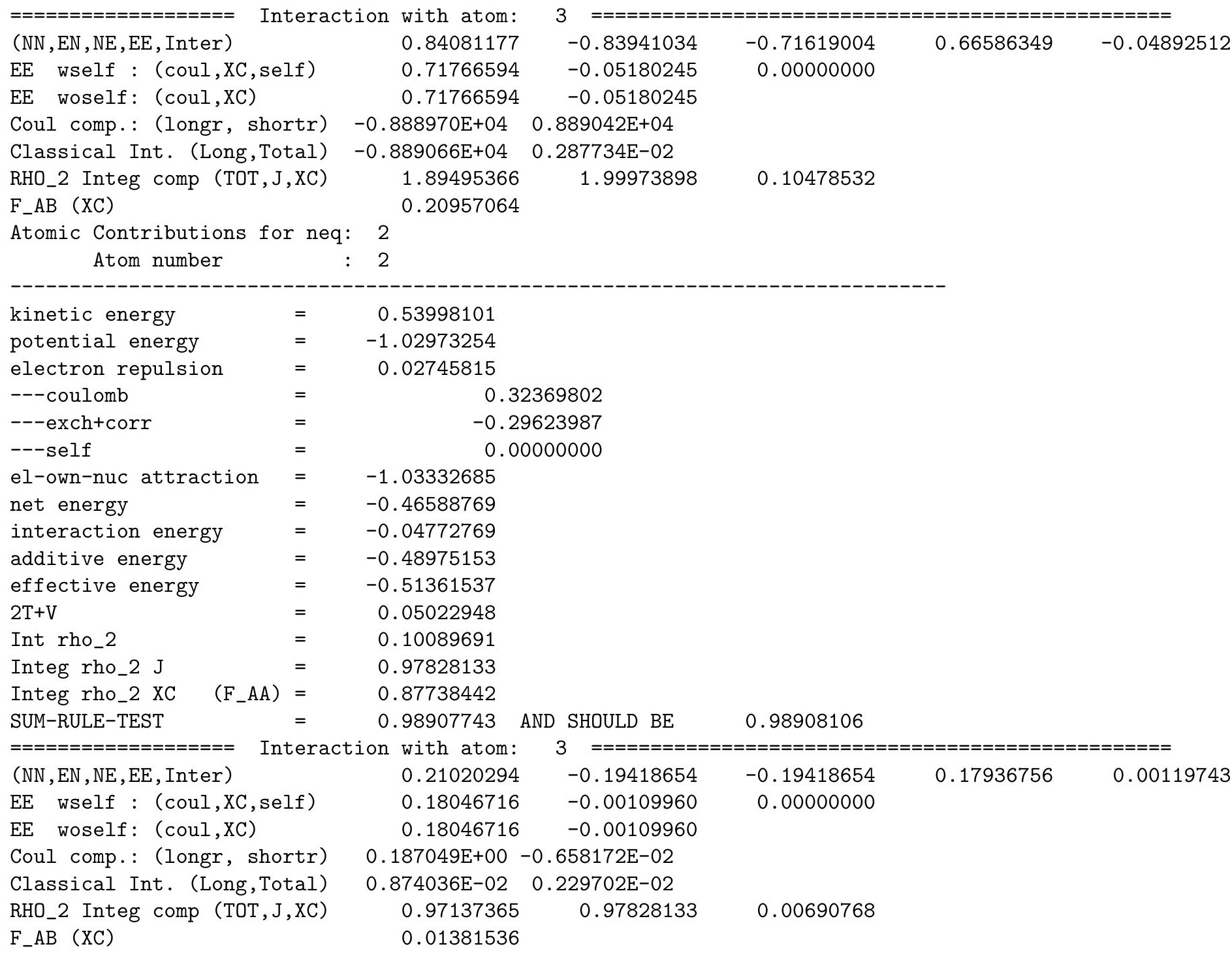

\# M-BASINS SPINLESS ELECTRON DISTRIBUTION FUNCTION

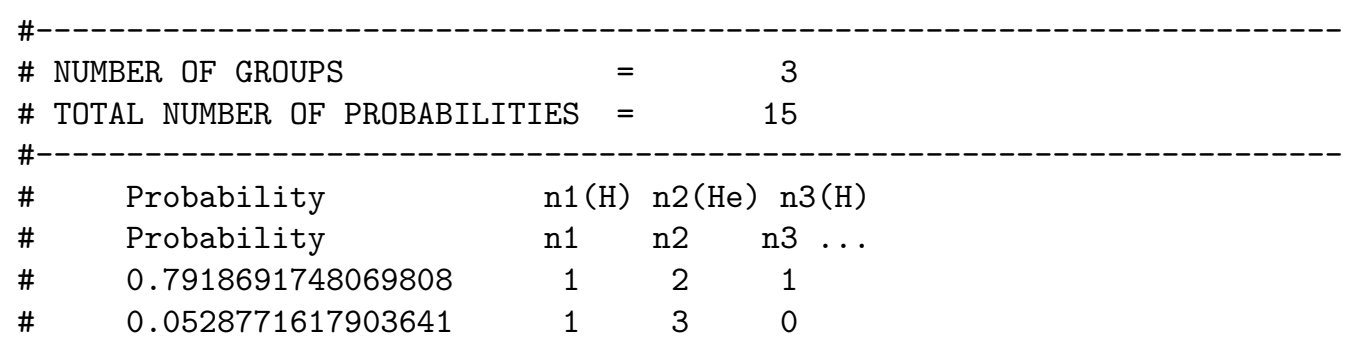




$\begin{array}{lllll}\# & 0.0528771617903641 & 0 & 3 & 1 \\ \# & 0.0439858313864634 & 2 & 1 & 1 \\ \# & 0.0439858313864634 & 1 & 1 & 2 \\ \# & 0.0051051647318951 & 2 & 2 & 0 \\ \# & 0.0051051647318951 & 0 & 2 & 2 \\ \# & 0.0030670864319878 & 0 & 4 & 0 \\ \# & 0.0006005387579096 & 2 & 0 & 2 \\ \# & 0.0001907003446540 & 3 & 0 & 1 \\ \# & 0.0001907003446540 & 1 & 0 & 3 \\ \# & 0.0000612673807049 & 3 & 1 & 0 \\ \# & 0.0000612673807049 & 0 & 1 & 3 \\ \# & 0.0000000781913864 & 4 & 0 & 0 \\ \# & 0.0000000781913864 & 0 & 0 & 4\end{array}$

Average populations and localization indices $\#\langle\mathrm{n}(\mathrm{1})\rangle=0.9890621540$ $\#\langle n(2)\rangle \quad=\quad 2.0217845225$

$\#\langle\mathrm{n}(3)\rangle \quad=\quad 0.9890621540$

$\#\langle\mathrm{n}(2) \mathrm{n}(1)\rangle=1.8949317902$

$\#\langle\mathrm{n}(3) \mathrm{n}(\mathrm{1})\rangle=0.9713588575$

$\#\langle\mathrm{n}(3) \mathrm{n}(\mathrm{2})\rangle=1.8949317902$

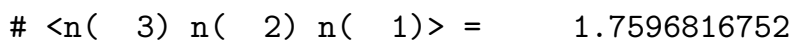

$\#$ delta $\left(\begin{array}{ll}1 & 1\end{array}\right) \quad=0.8773481301$

\# delta_( $\left.\begin{array}{ll}2 & 2\end{array}\right) \quad=1.8121226684$

$\%$ Localization $=88.7051$

\# delta_( 33$)$

3) $=0.8773481301$

$\%$ Localization $=89.6299$

$\%$ Localization $=88.7051$

Delocalization indices

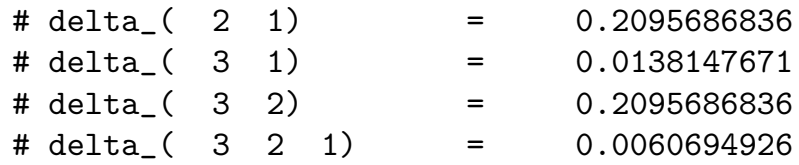

\# Fragment A formed by atoms 1: He

\# Fragment B formed by atoms 2 3: H, H

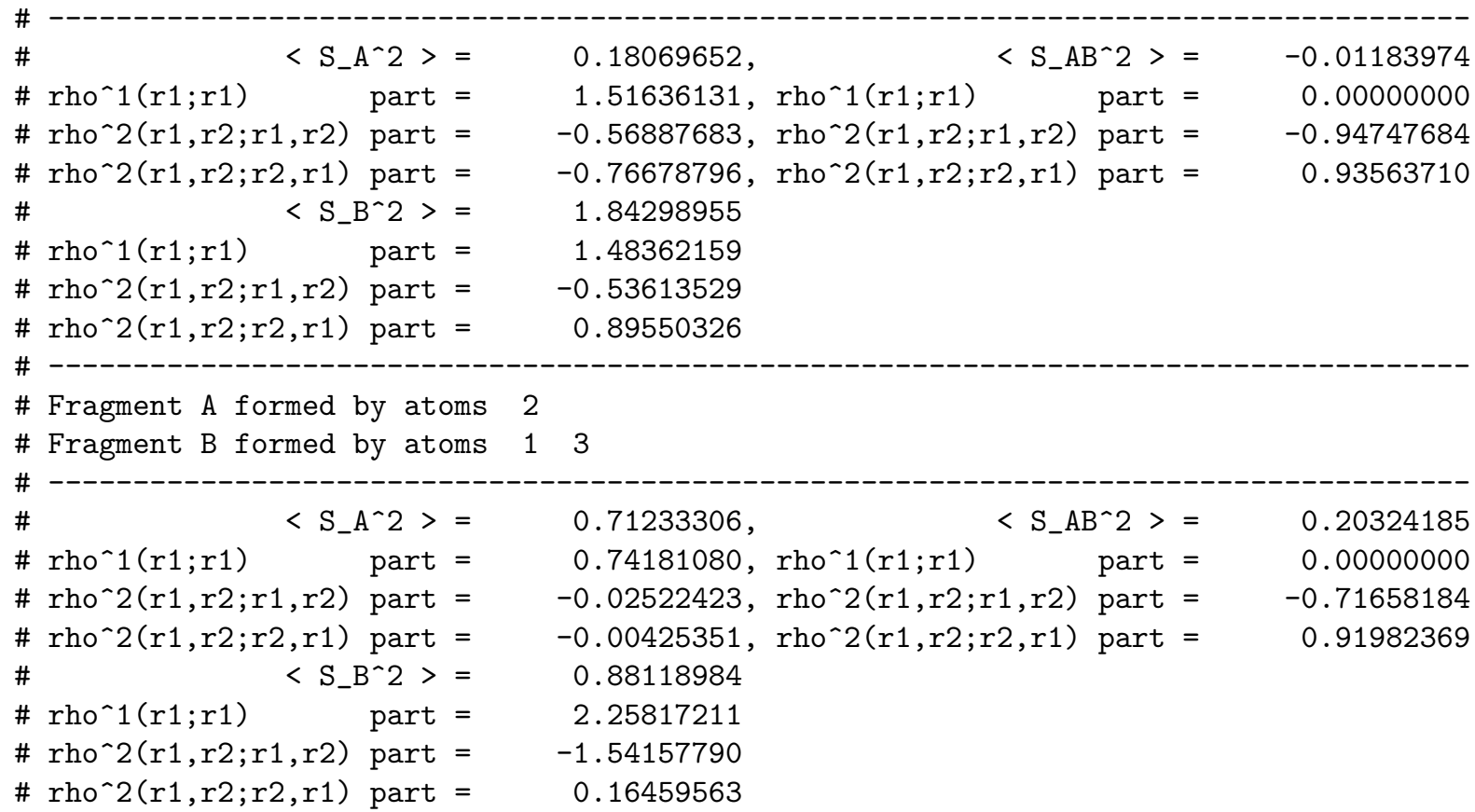




\subsection{H-He-H BS}

\begin{tabular}{lcccc} 
ATOM & ATOMIC & \multicolumn{3}{c}{ COORDINATES (BOHR) } \\
& CHARGE & $\mathrm{X}$ & $\mathrm{Y}$ & $\mathrm{Z}$ \\
$\mathrm{HE}$ & 2.0 & 0.0000000000 & 0.0000000000 & 1.6629588692 \\
$\mathrm{H}$ & 1.0 & 0.0000000000 & -1.7007533889 & 0.0000000000 \\
$\mathrm{H}$ & 1.0 & 0.0000000000 & 1.7007533889 & 0.0000000000
\end{tabular}

EIGENVECTORS

$\begin{array}{ccccc}1 & 2 & 3 & 4 & 5 \\ -1.0754 & -0.2854 & -0.0639 & 0.3661 & 0.3980 \\ \text { A1 } & \text { B2 } & \text { A1 } & \text { A1 } & \text { B2 }\end{array}$

NATURAL ORBITALS IN ATOMIC ORBITAL BASIS

$\begin{array}{ccccc}1 & 2 & 3 & 4 & 5 \\ 1.9837 & 1.1700 & 0.8282 & 0.0069 & 0.0028 \\ \text { A1 } & \text { A1 } & \text { B2 } & \text { A1 } & \text { B2 }\end{array}$

TOTAL ENERGY $=\quad-3.8102031609$

ELECTRON-ELECTRON POTENTIAL ENERGY =

2.7273896090

NUCLEUS-ELECTRON POTENTIAL ENERGY =

$-12.6211262778$

NUCLEUS-NUCLEUS POTENTIAL ENERGY =

1.9756107487

TOTAL POTENTIAL ENERGY =

COEFFICIENT/ OCCUPIED ACTIVE SPIN ORBITALS
$0.7359975185 \mathrm{E}+00$
$\begin{array}{llll}1 & 3 & -1 & -3\end{array}$
$\begin{array}{lllll}-0.6288086818 E+00 & 1 & 2 & -1 & -2\end{array}$
$-0.1336656226 \mathrm{E}+00 \quad 1 \quad 3 \quad-1 \quad-4$
$-0.1336656226 \mathrm{E}+00 \quad 1 \quad 4 \quad-1 \quad-3$
$\begin{array}{lllll}0.6975657511 \mathrm{E}-01 & 1 & 2 & -1 & -5\end{array}$
$\begin{array}{lllll}0.6975657511 \mathrm{E}-01 & 1 & 5 & -1 & -2\end{array}$
$\begin{array}{lllll}0.3673587766 \mathrm{E}-01 & 1 & 2 & -2 & -3\end{array}$
$\begin{array}{lllll}0.3673587766 \mathrm{E}-01 & 2 & 3 & -1 & -2\end{array}$
$-0.2731375808 \mathrm{E}-01 \quad 3 \quad 6 \quad-3 \quad-6$
$\begin{array}{lllll}0.2590922013 \mathrm{E}-01 & 2 & 6 & -2 & -6\end{array}$
$\begin{array}{lllll}-0.2479618149 \mathrm{E}-01 & 1 & 2 & -1 & -9\end{array}$
$\begin{array}{lllll}-0.2479618149 \mathrm{E}-01 & 1 & 9 & -1 & -2\end{array}$
$-0.2038016853 E-01 \quad 1 \quad 3 \quad-1 \quad-6$
$-0.2038016853 \mathrm{E}-01 \quad 1 \quad 6 \quad-1 \quad-3$
$0.1822969654 \mathrm{E}-01 \quad 1 \quad 4 \quad-1 \quad-4$
$\begin{array}{lllll}-0.1756747238 \mathrm{E}-01 & 3 & 7 & -3 & -7\end{array}$
$\begin{array}{lllll}-0.1702480265 E-01 & 1 & 5 & -2 & -3\end{array}$
$\begin{array}{lllll}-0.1702480265 \mathrm{E}-01 & 2 & 3 & -1 & -5\end{array}$
$\begin{array}{lllll}-0.1569720429 \mathrm{E}-01 & 3 & 9 & -3 & -9\end{array}$
$\begin{array}{lllll}0.1469947170 \mathrm{E}-01 & 2 & 7 & -2 & -7\end{array}$
$\begin{array}{lllll}-0.1412878076 \mathrm{E}-01 & 2 & 3 & -2 & -6\end{array}$
$\begin{array}{lllll}-0.1412878076 \mathrm{E}-01 & 2 & 6 & -2 & -3\end{array}$

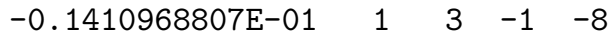
$\begin{array}{lllll}-0.1410968807 \mathrm{E}-01 & 1 & 8 & -1 & -3\end{array}$
$\begin{array}{lllll}0.1347012778 \mathrm{E}-01 & 2 & 9 & -2 & -9\end{array}$
$\begin{array}{lllll}-0.1308559822 E-01 & 3 & 8 & -3 & -8\end{array}$
$\begin{array}{llllll}0.1288258920 \mathrm{E}-01 & 1 & 6 & -3 & -6\end{array}$

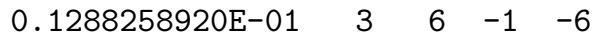
$0.1248318944 \mathrm{E}-01 \quad 1 \quad 3 \quad-1 \quad-15$
$\begin{array}{llllll}0.1248318944 \mathrm{E}-01 & 1 & 15 & -1 & -3\end{array}$ 


$\begin{array}{rrrrr}0.1072337844 \mathrm{E}-01 & 2 & 8 & -2 & -8 \\ -0.1055932944 \mathrm{E}-01 & 1 & 2 & -1 & -17 \\ -0.1055932944 \mathrm{E}-01 & 1 & 17 & -1 & -2 \\ -0.1043358411 \mathrm{E}-01 & 3 & 7 & -3 & -12 \\ -0.1043358411 \mathrm{E}-01 & 3 & 12 & -3 & -7 \\ 0.1030524333 \mathrm{E}-01 & 1 & 3 & -1 & -16 \\ 0.1030524333 \mathrm{E}-01 & 1 & 16 & -1 & -3 \\ -0.1007802751 \mathrm{E}-01 & 3 & 18 & -3 & -18 \\ -0.1007698826 \mathrm{E}-01 & 1 & 3 & -3 & -6 \\ -0.1007698826 \mathrm{E}-01 & 3 & 6 & -1 & -3\end{array}$

Atomic Contributions for neq: 1

Atom number : 1

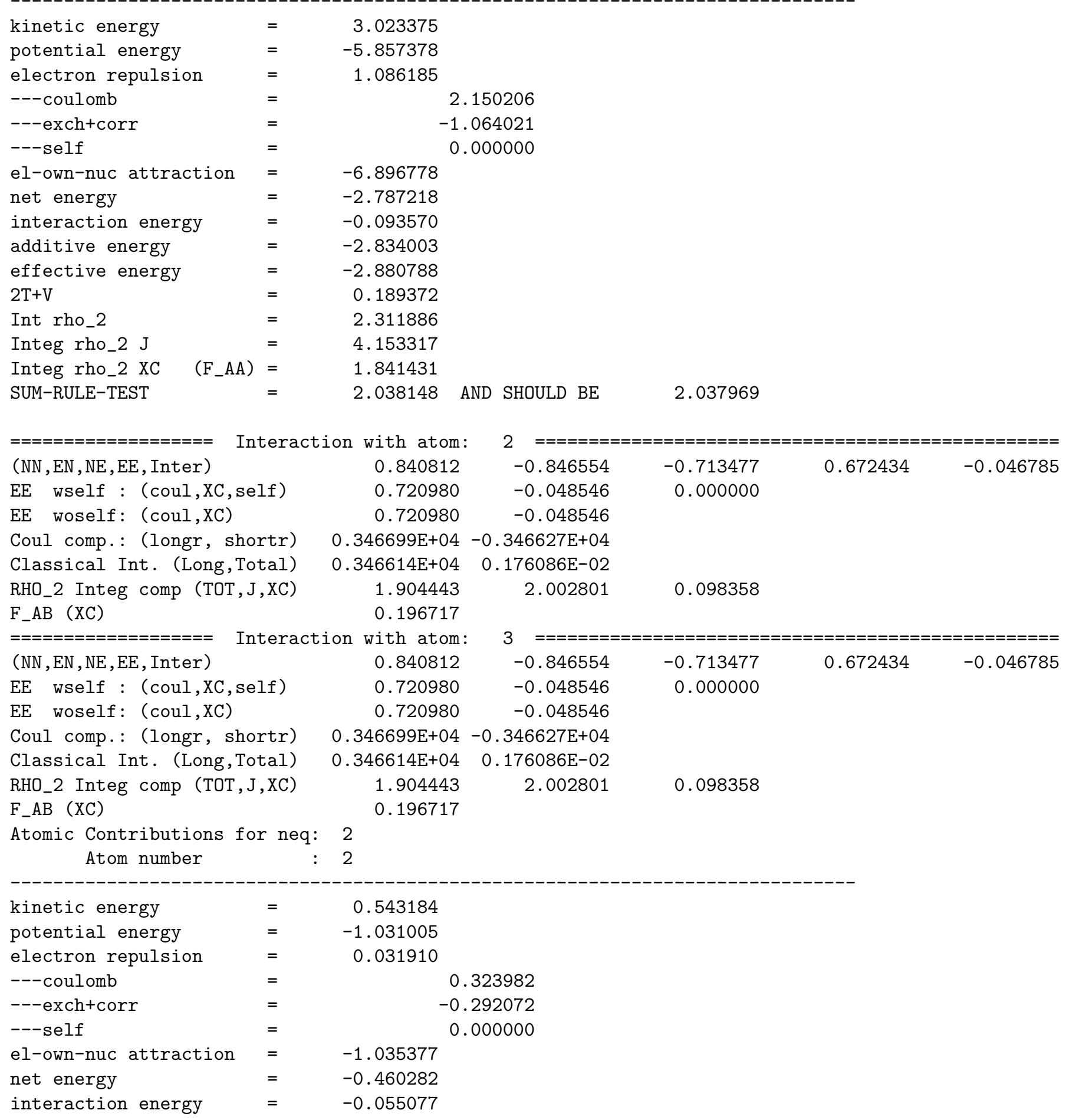




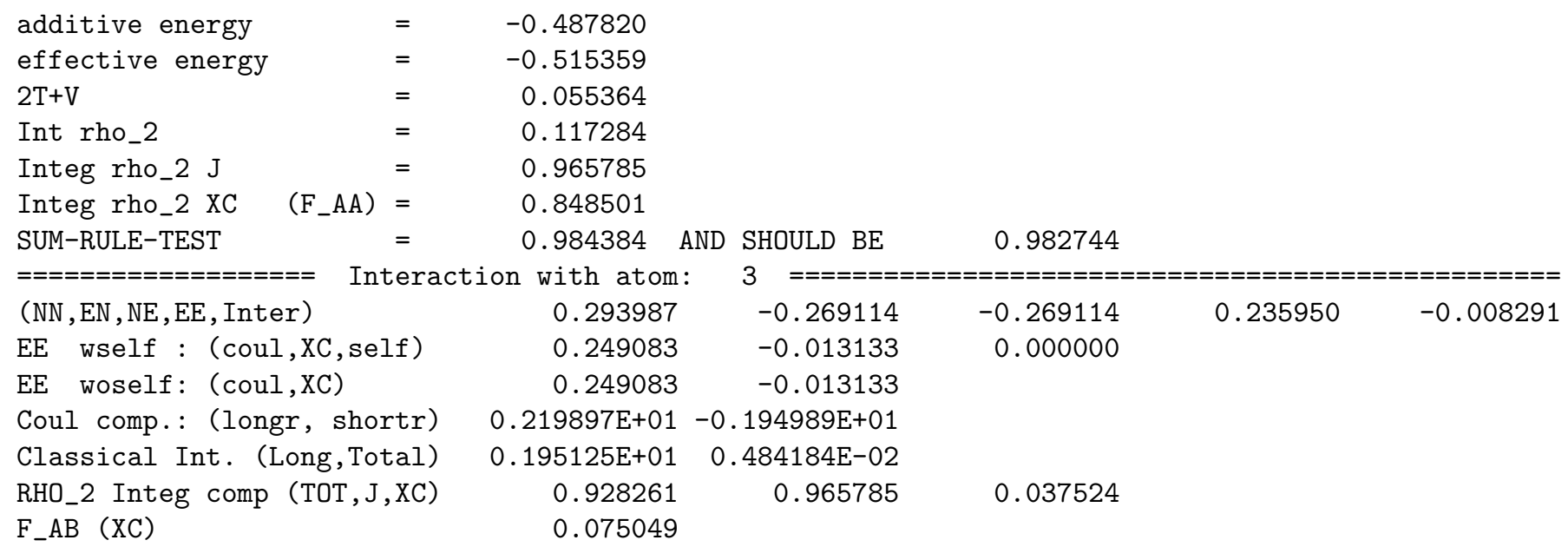

\# M-BASINS SPINLESS ELECTRON DISTRIBUTION FUNCTION

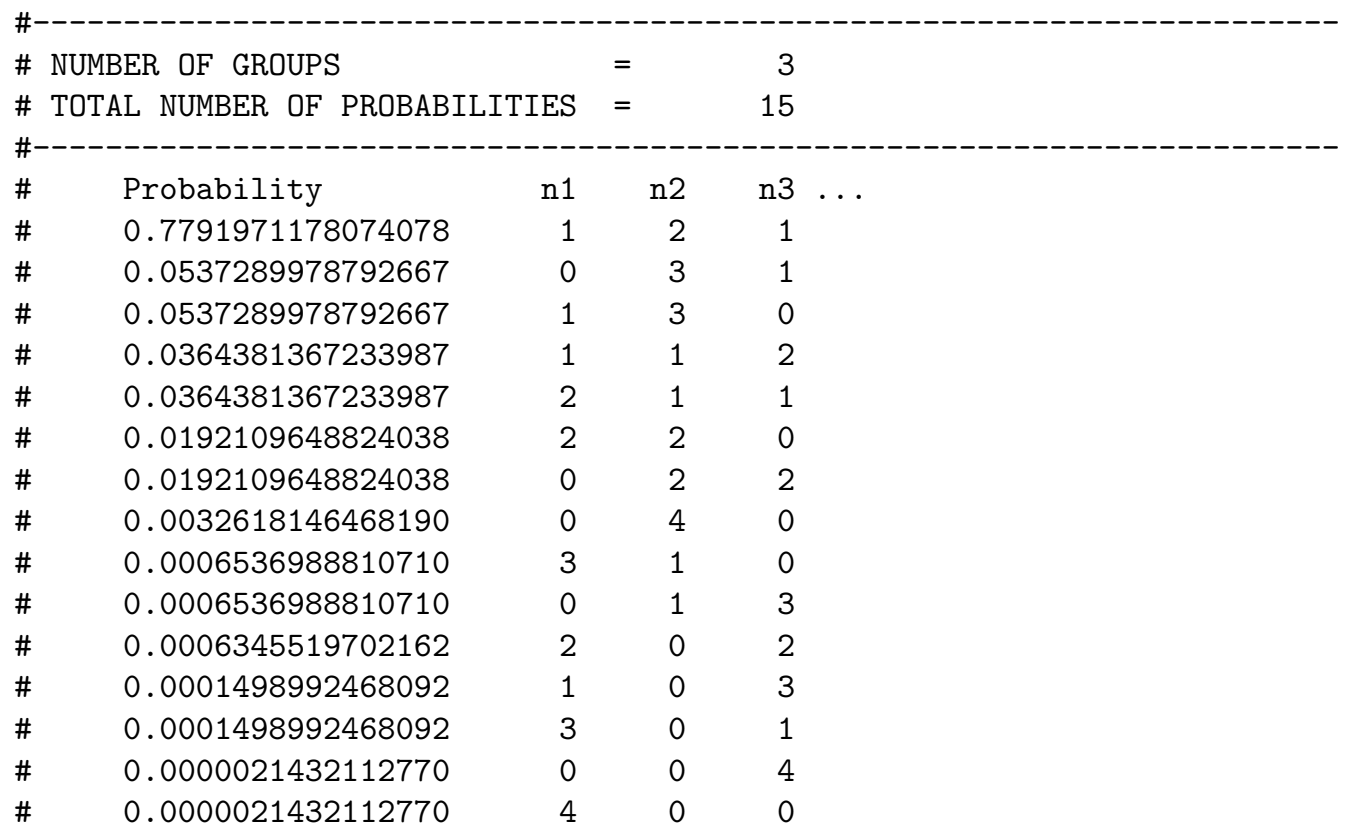

Average populations and delocalization indices

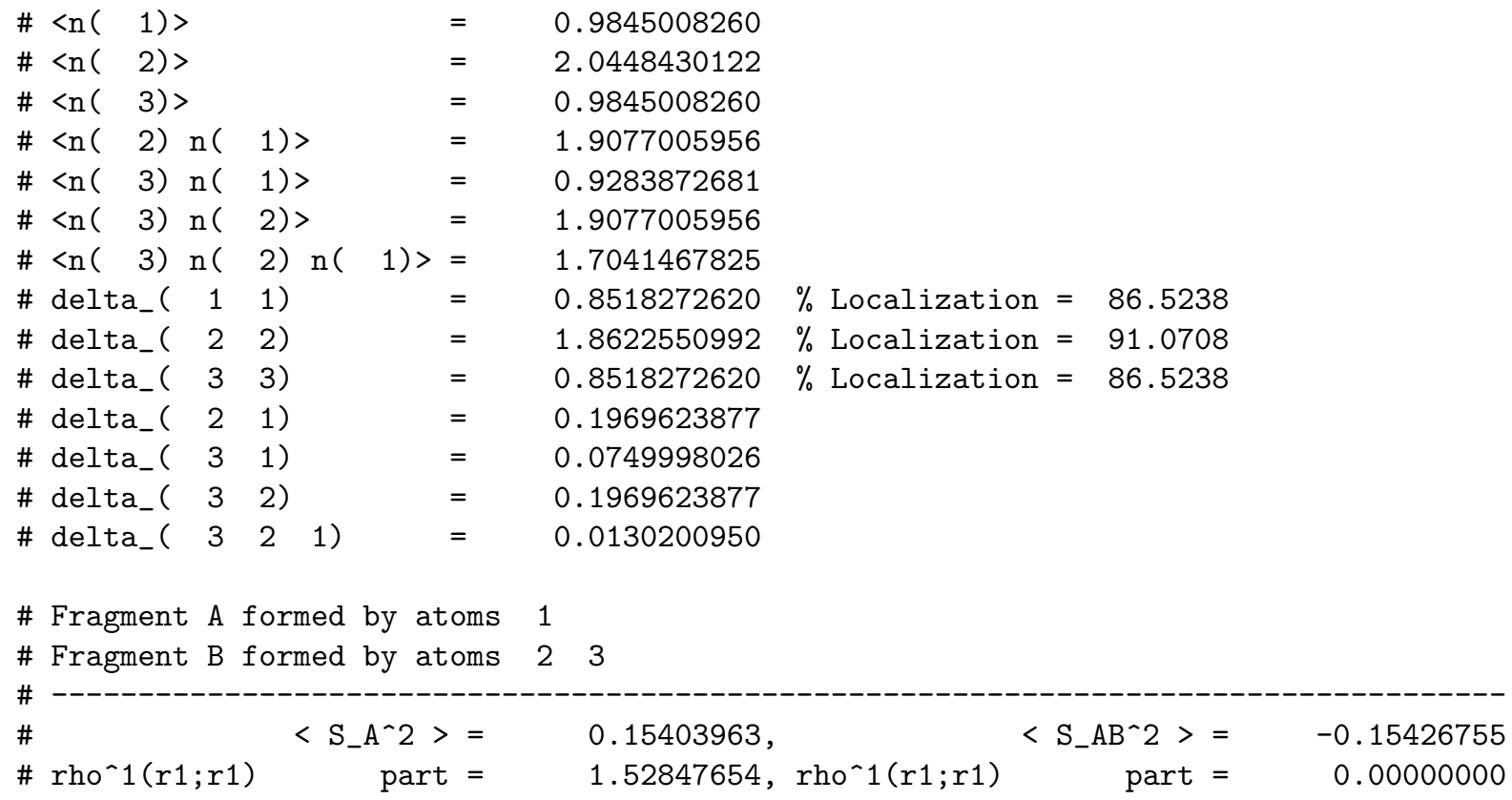


\# rho^2(r1,r2;r1,r2) part =

\# rho^2(r1,r2;r2,r1) part =

\#

\# rho^1(r1;r1)

$<\mathrm{S}_{-} \mathrm{B}^{\wedge} 2>=$

$r h 0^{\wedge} 2(r 1, r 2 ; r 1, r 2)$ part $=$

\# rho^2(r1,r2;r2,r1) part =

\#

\# Fragment A formed by atoms 2

\# Fragment B formed by atoms 13

\#

\# $\quad<\mathrm{S}_{-} \mathrm{A}^{\sim} 2>=$

\# rho^1(r1;r1) part =

\# rho^2(r1,r2;r1,r2) part =

\# rho^2(r1,r2;r2,r1) part =

\#

\# rho^1(r1;r1)

$<\mathrm{S} \_\mathrm{B}^{\wedge} 2>=$

part $=$

\# rho^2(r1,r2;r1,r2) part =

\# rho^2(r1,r2;r2,r1) part =
$-0.57797140, r h 0^{\wedge} 2(r 1, r 2 ; r 1, r 2)$ part $=$

-0.79646551 , rho^2(r1,r2;r2,r1) part =

0.15189605

1.47411564

$-0.52277271$

$-0.79944689$

$\begin{array}{rrr}0.69033136, & <S_{-} A^{\wedge} 2>= \\ 0.73705782, & r h 0^{\wedge} 1(r 1 ; r 1) & \text { part }= \\ -0.02932109, & r h 0^{\wedge} 2(r 1, r 2 ; r 1, r 2) & \text { part }= \\ -0.01740537, & r h 0^{\wedge} 2(r 1, r 2 ; r 2, r 1) & \text { part }= \\ 0.69010344 & & \\ 2.26553437 & & \\ -1.55951386 & & \\ -0.01591707 & \end{array}$

$-0.69151711$

0.00000000

$-0.70817594$

0.01665884

\subsection{H-He-H BT}

\begin{tabular}{|c|c|c|c|c|c|c|}
\hline \multirow[t]{2}{*}{ ATOM } & ATOMIC & \multicolumn{5}{|c|}{ COORDINATES (BOHR) } \\
\hline & CHARGE & \multicolumn{2}{|c|}{$\mathrm{X}$} & \multicolumn{2}{|l|}{$\mathrm{Y}$} & Z \\
\hline $\mathrm{HE}$ & 2.0 & \multicolumn{2}{|c|}{0.0000000000} & \multicolumn{2}{|c|}{0.0000000000} & 1.6629588692 \\
\hline $\mathrm{H}$ & 1.0 & \multicolumn{2}{|c|}{0.0000000000} & \multicolumn{2}{|c|}{-1.7007533889} & 0.0000000000 \\
\hline \multirow[t]{7}{*}{$\mathrm{H}$} & 1.0 & \multicolumn{2}{|c|}{0.0000000000} & \multicolumn{2}{|c|}{1.7007533889} & 0.0000000000 \\
\hline & -------1 & -- & & & & \\
\hline & \multicolumn{6}{|c|}{ EIGENVECTORS } \\
\hline & \multicolumn{6}{|c|}{------------ } \\
\hline & & 1 & 2 & 3 & 4 & 5 \\
\hline & & -0.9526 & -0.1585 & -0.1286 & 0.2929 & 0.3222 \\
\hline & & $\mathrm{A} 1$ & $\mathrm{~A} 1$ & B2 & $\mathrm{A} 1$ & B2 \\
\hline
\end{tabular}

NATURAL ORBITALS IN ATOMIC ORBITAL BASIS

$\begin{array}{ccccc}1 & 2 & 3 & 4 & 5 \\ 1.9830 & 0.9993 & 0.9989 & 0.0071 & 0.0028 \\ \mathrm{~A} 1 & \mathrm{~A} 1 & \mathrm{~B} 2 & \mathrm{~A} 1 & \mathrm{~A} 1\end{array}$

TOTAL ENERGY $=\quad-3.8143631935$

ELECTRON-ELECTRON POTENTIAL ENERGY =

2.7116381747

NUCLEUS-ELECTRON POTENTIAL ENERGY =

$-12.5804293330$

NUCLEUS-NUCLEUS POTENTIAL ENERGY =

1.9756107487

TOTAL POTENTIAL ENERGY =

$-7.8931804096$ TOTAL KINETIC ENERGY =

4.0788172161

VIRIAL RATIO $(\mathrm{V} / \mathrm{T})=$

1.9351640418

COEFFICIENT/ OCCUPIED ACTIVE SPIN ORBITALS
$\begin{array}{lllll}0.7031431599 \mathrm{E}+00 & 1 & 2 & -1 & -3\end{array}$
$\begin{array}{lllll}-0.7031431599 \mathrm{E}+00 & 1 & 3 & -1 & -2\end{array}$
$\begin{array}{lllll}-0.2764695850 \mathrm{E}-01 & 2 & 6 & -3 & -6\end{array}$
$0.2764695850 \mathrm{E}-01 \quad 3 \quad 6 \quad-2 \quad-6$
$0.1659919446 \mathrm{E}-01 \quad 2 \quad 3 \quad-2 \quad-6$
$\begin{array}{lllll}-0.1659919446 \mathrm{E}-01 & 2 & 6 & -2 & -3\end{array}$
$\begin{array}{lllll}-0.1495770357 \mathrm{E}-01 & 1 & 4 & -2 & -3\end{array}$ 


$\begin{array}{rrrrr}0.1495770357 \mathrm{E}-01 & 2 & 3 & -1 & -4 \\ -0.1417148789 \mathrm{E}-01 & 2 & 9 & -3 & -9 \\ 0.1417148789 \mathrm{E}-01 & 3 & 9 & -2 & -9 \\ -0.1367153292 \mathrm{E}-01 & 2 & 8 & -3 & -8 \\ 0.1367153292 \mathrm{E}-01 & 3 & 8 & -2 & -8 \\ -0.1348775998 \mathrm{E}-01 & 1 & 2 & -2 & -3 \\ 0.1348775998 \mathrm{E}-01 & 2 & 3 & -1 & -2 \\ 0.1127877017 \mathrm{E}-01 & 2 & 13 & -3 & -8 \\ -0.1127877017 \mathrm{E}-01 & 3 & 8 & -2 & -13 \\ 0.1126510053 \mathrm{E}-01 & 2 & 8 & -3 & -13 \\ -0.1126510053 \mathrm{E}-01 & 3 & 13 & -2 & -8 \\ -0.1113156367 \mathrm{E}-01 & 2 & 7 & -3 & -7 \\ 0.1113156367 \mathrm{E}-01 & 3 & 7 & -2 & -7 \\ -0.1092395670 \mathrm{E}-01 & 2 & 13 & -3 & -13 \\ 0.1092395670 \mathrm{E}-01 & 3 & 13 & -2 & -13 \\ 0.1073820145 \mathrm{E}-01 & 2 & 3 & -2 & -7 \\ -0.1073820145 \mathrm{E}-01 & 2 & 7 & -2 & -3 \\ 0.1017998312 \mathrm{E}-01 & 1 & 7 & -2 & -3 \\ -0.1017998312 \mathrm{E}-01 & 2 & 3 & -1 & -7 \\ 0.1016883214 \mathrm{E}-01 & 1 & 6 & -3 & -6 \\ -0.1016883214 \mathrm{E}-01 & 3 & 6 & -1 & -6\end{array}$

Atomic Contributions for neq: 1 Atom number

: 1

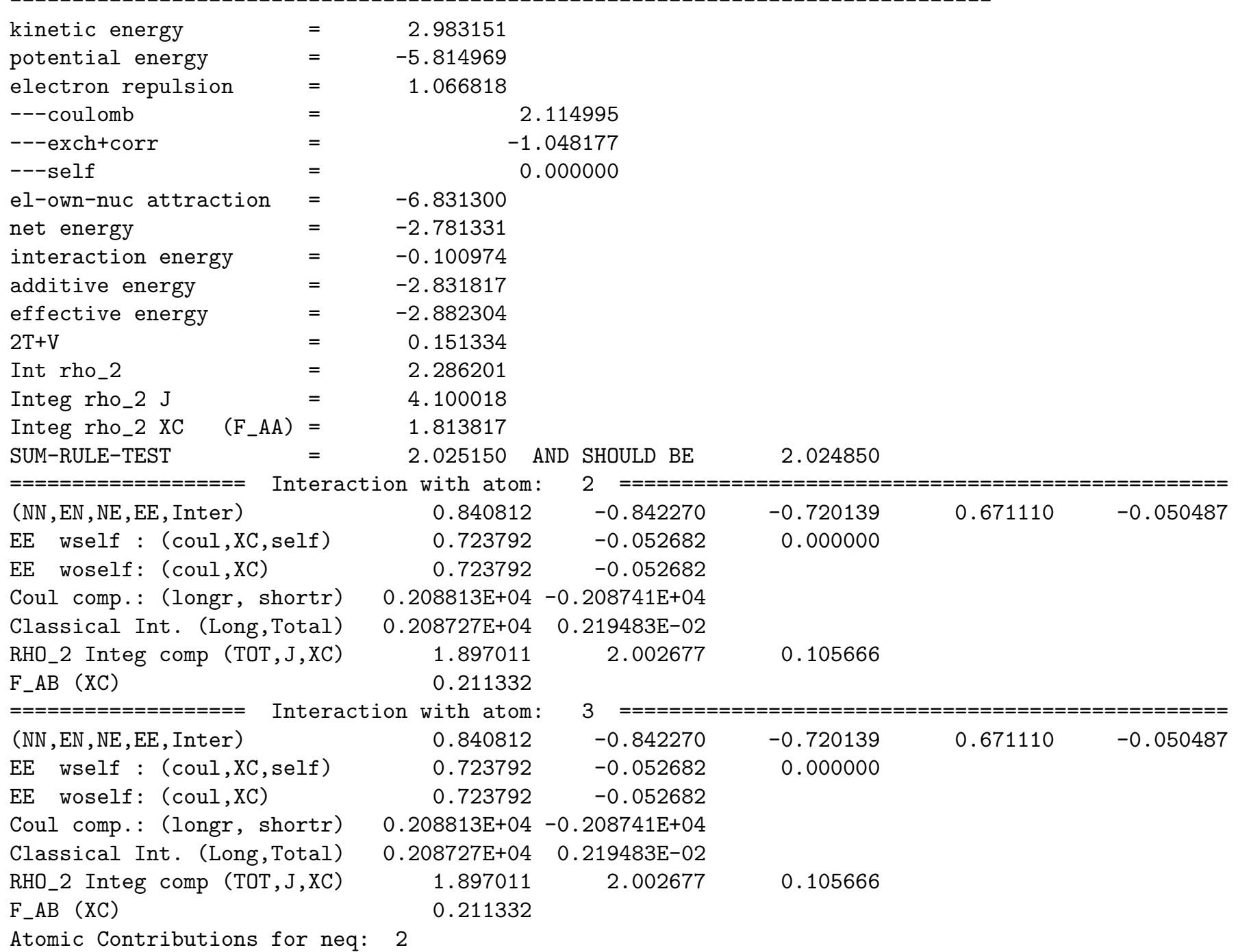




\begin{tabular}{|c|c|c|c|c|c|}
\hline kinetic energy & $=$ & 0.548753 & & & \\
\hline potential energy & $=$ & -1.039705 & & & \\
\hline electron repulsion & $=$ & 0.033061 & & & \\
\hline--- coulomb & $=$ & 0.328917 & & & \\
\hline$---e x c h+\operatorname{corr}$ & $=$ & -0.295856 & & & \\
\hline$---\operatorname{self}$ & $=$ & 0.000000 & & & \\
\hline el-own-nuc attraction & $=$ & -1.044443 & & & \\
\hline net energy & $=$ & -0.462630 & & & \\
\hline interaction energy & $=$ & -0.056645 & & & \\
\hline additive energy & $=$ & -0.490952 & & & \\
\hline effective energy & $=$ & -0.519275 & & & \\
\hline $2 \mathrm{~T}+\mathrm{V}$ & $=$ & 0.057800 & & & \\
\hline Int rho_2 & $=$ & 0.124451 & & & \\
\hline Integ rho_2 J & $=$ & 0.978219 & & & \\
\hline Integ rho_2 XC & $=$ & 0.853768 & & & \\
\hline SUM-RULE-TEST & $=$ & 0.990378 AND SHOULD BE & 0.989049 & & \\
\hline$===================\mathrm{In}$ & terac & ion with atom: & $==========$ & $==========$ & $=========$ \\
\hline (NN, EN, NE, EE, Inter) & & -0.269720 & -0.269720 & 0.239295 & -0.006158 \\
\hline EE wself : (coul, XC, se & lf) & -0.010748 & 0.000000 & & \\
\hline EE woself: (coul, XC) & & -0.010748 & & & \\
\hline Coul comp.: (longr, sho & rtr) & $0.159259 \mathrm{E}+01-0.134255 \mathrm{E}+01$ & & & \\
\hline Classical Int. (Long, To & tal) & $0.134420 \mathrm{E}+01 \quad 0.458975 \mathrm{E}-02$ & & & \\
\hline RHO_2 Integ comp (TOT, J & $, \mathrm{XC})$ & 0.978219 & 0.030944 & & \\
\hline$F_{-} A B \quad(X C)$ & & 0.061888 & & & \\
\hline
\end{tabular}

\# M-BASINS SPINLESS ELECTRON DISTRIBUTION FUNCTION

\begin{tabular}{|c|c|c|c|c|}
\hline \multicolumn{3}{|c|}{ \# NUMBER OF GROUPS } & $=$ & 3 \\
\hline \multicolumn{3}{|c|}{ \# TOTAL NUMBER OF PROBABILITIES } & $=$ & 15 \\
\hline \# & Probability & $\mathrm{n} 1$ & $\mathrm{n} 2$ & n3 $\ldots$ \\
\hline \# & 0.7713682927334732 & 1 & 2 & 1 \\
\hline \# & 0.0536763809816437 & 0 & 3 & 1 \\
\hline \# & 0.0536763809816437 & 1 & 3 & 0 \\
\hline \# & 0.0432743923083669 & 1 & 1 & 2 \\
\hline$\#$ & 0.0432743923083669 & 2 & 1 & 1 \\
\hline \# & 0.0160798162434760 & 0 & 2 & 2 \\
\hline$\#$ & 0.0160798162434760 & 2 & 2 & 0 \\
\hline$\#$ & 0.0034020001643794 & 0 & 4 & 0 \\
\hline$\#$ & 0.0006255404391828 & 0 & 1 & 3 \\
\hline$\#$ & 0.0006255404391828 & 3 & 1 & 0 \\
\hline$\#$ & 0.0004993336137804 & 2 & 0 & 2 \\
\hline$\#$ & 0.0001814183507976 & 3 & 0 & 1 \\
\hline$\#$ & 0.0001814183507976 & 1 & 0 & 3 \\
\hline \# & 0.0000022621629189 & 0 & 0 & 4 \\
\hline$\#$ & 0.0000022621629189 & 4 & 0 & 0 \\
\hline \multicolumn{5}{|c|}{ \#--------------- } \\
\hline $\begin{array}{l}\# \\
\#\end{array}$ & $\begin{array}{l}1.0029492474844048 \\
1.0029492474844048\end{array}$ & $\begin{array}{l}<-- \text { SUM } \\
<--- \text { TOT }\end{array}$ & $\begin{array}{r}15 \\
\text { SUM }\end{array}$ & PROBABILITIES >0.0000000000E+00 \\
\hline
\end{tabular}

Average populations and delocalization indices

\begin{tabular}{|c|c|c|c|}
\hline$\#<\mathrm{n}($ & 1) $>$ & $=$ & 0.9906374937 \\
\hline$\#<\mathrm{n}$ & 2) $>$ & $=$ & 2.0305220025 \\
\hline \# & 3) $>$ & $=$ & 0.99063749 \\
\hline . & 2) $n(1)>$ & $=$ & 1.8 \\
\hline
\end{tabular}




\begin{tabular}{|c|c|c|c|c|c|c|c|}
\hline$\#<\mathrm{n}(3)$ & $\mathrm{n}($ & 1) $>$ & & $=$ & 0.9475517065 & & \\
\hline$\#<n\left(\begin{array}{l}3 \\
*\end{array}\right)$ & $\mathrm{n}($ & 2) > & & $=$ & 1.8997847916 & & \\
\hline$\#<n\left(\begin{array}{l}3 \\
*\end{array}\right)$ & $\mathrm{n}($ & 2) $\mathrm{n}($ & 1) $>$ & $=$ & 1.7158341547 & & \\
\hline$\#$ delta_( & 1 & 1) & & $=$ & 0.8567866610 & $\%$ Localization = & 86.4884 \\
\hline \# delta_( & 2 & 2) & & $=$ & 1.8310231784 & $\%$ Localization = & 90.1750 \\
\hline$\#$ delta_( & 3 & 3) & & $=$ & 0.8567866610 & $\%$ Localization = & 86.4884 \\
\hline \# delta_( & 2 & 1) & & $=$ & 0.2115879829 & & \\
\hline$\#$ delta_( & 3 & 1) & & $=$ & 0.0618333123 & & \\
\hline \# delta_( & 3 & 2) & & $=$ & 0.2115879829 & & \\
\hline$\#$ delta_( & 3 & $21)$ & & $=$ & 0.0145869141 & & \\
\hline
\end{tabular}

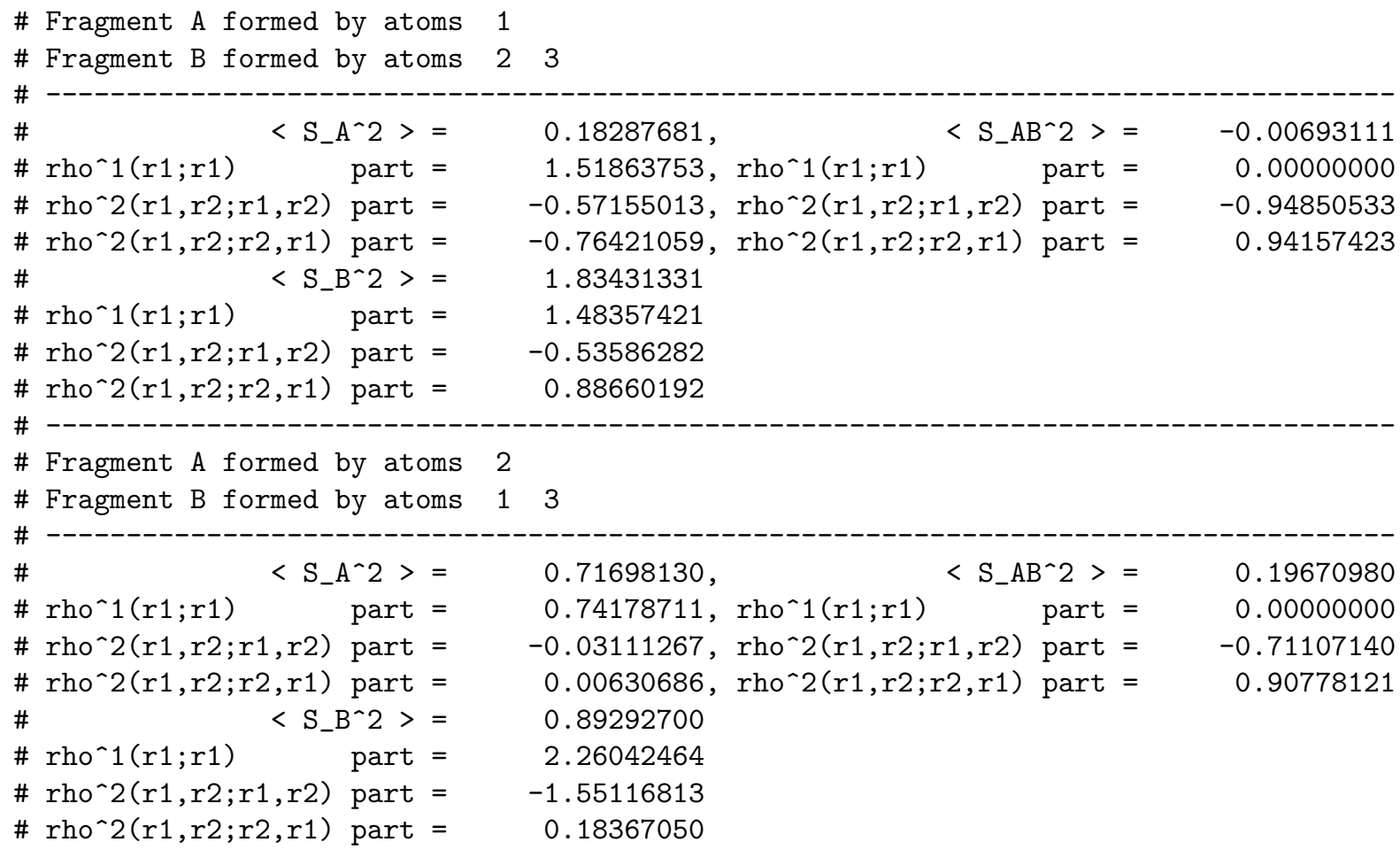

\subsection{ECP H-He-H LS}

Geometries as in the non-ECP calculations. With only two electrons, the EDF and its statistics is immediately found from the delocalization index $\left(\mathrm{F}_{\_} \mathrm{AB}\right)$ as follows: $p(2,0)=p(0,2)=(1-p(1,1)) / 2=4 \delta^{A B}$. Similarly, the local spin is given by $\left\langle S_{A}^{2}\right\rangle=3 / 4 p(1,1)$.

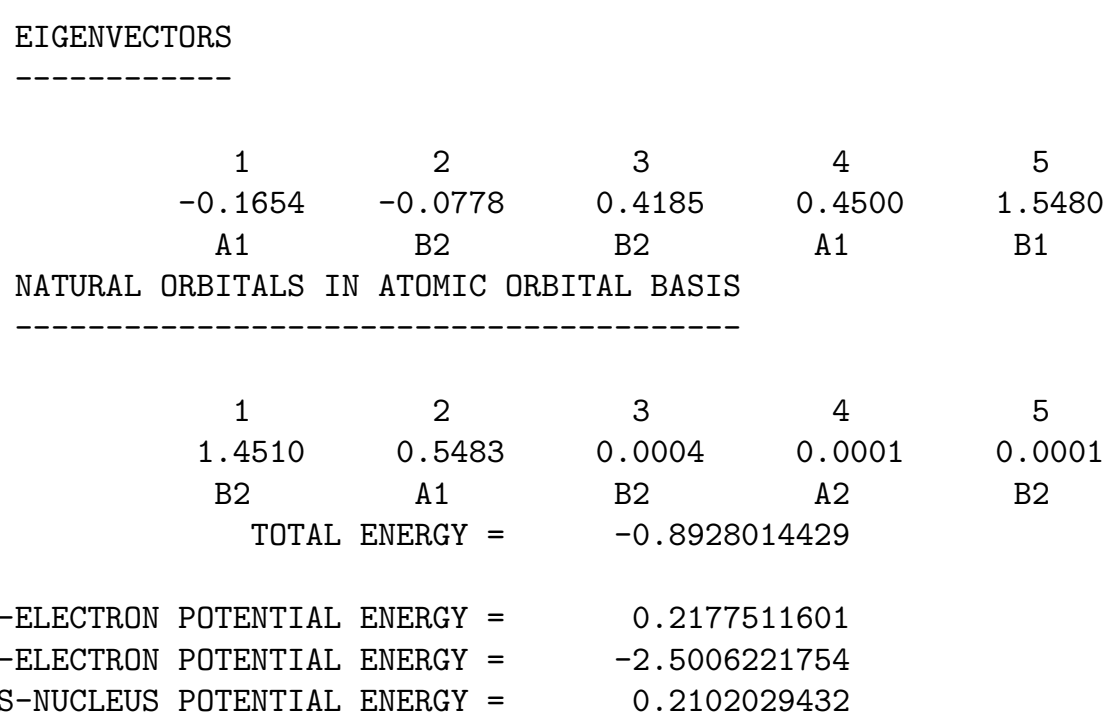




$\begin{array}{rr}\text { TOTAL POTENTIAL ENERGY }= & -2.0726680720 \\ \text { TOTAL KINETIC ENERGY }= & 1.1798666291 \\ \text { VIRIAL RATIO }(\mathrm{V} / \mathrm{T})= & 1.7566969189\end{array}$

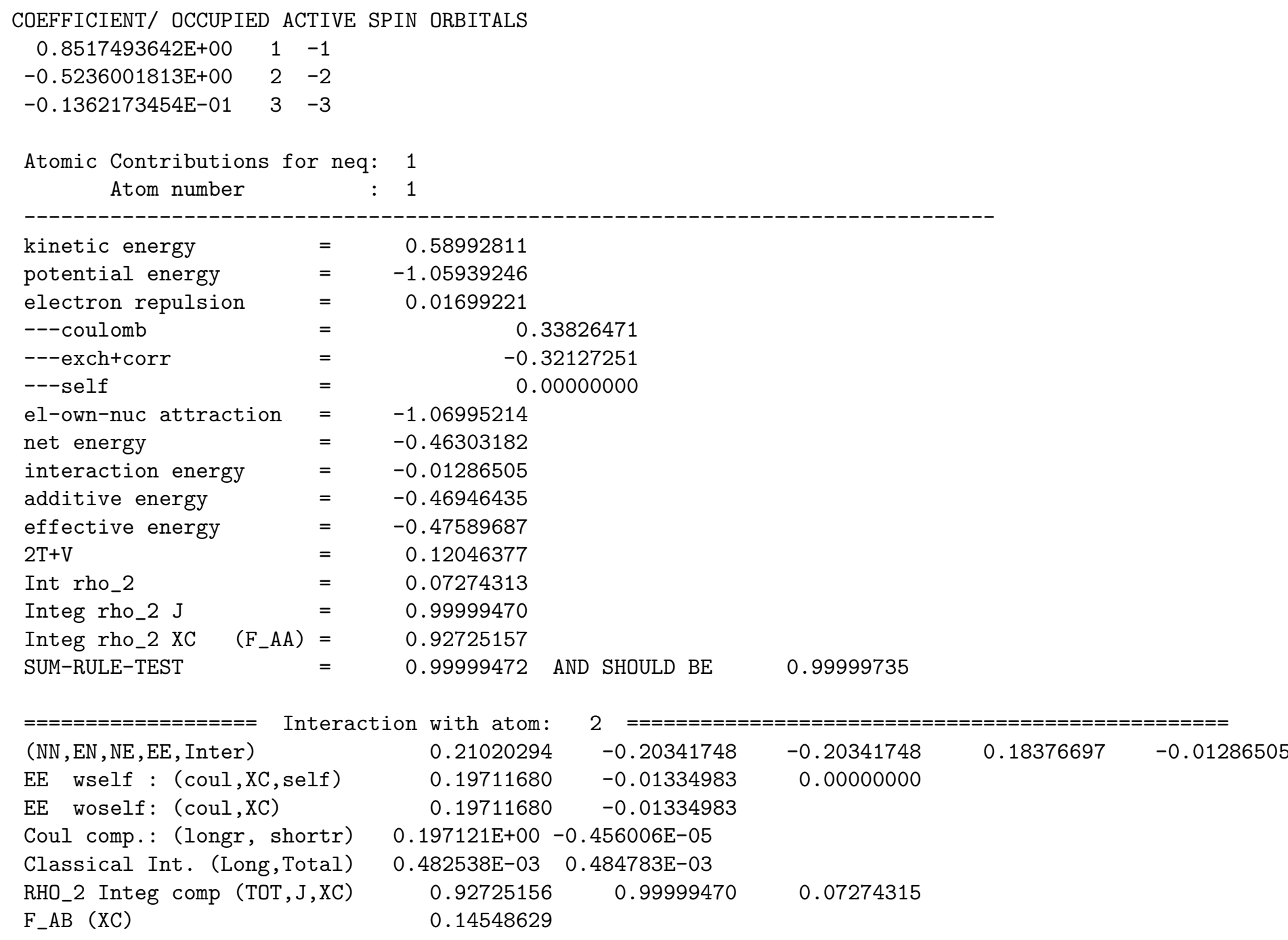

\subsection{ECP H-He-H LT}

EIGENVECTORS

$\begin{array}{ccccc}1 & 2 & 3 & 4 & 5 \\ -0.4836 & -0.3925 & 0.3699 & 0.4175 & 1.5099 \\ \text { B2 } & \text { A1 } & \text { B2 } & \text { A1 } & \text { A1 }\end{array}$

NATURAL ORBITALS IN ATOMIC ORBITAL BASIS

$\begin{array}{ccccc}1 & 2 & 3 & 4 & 5 \\ 1.0000 & 1.0000 & 0.0000 & 0.0000 & 0.0000 \\ \mathrm{~A} 1 & \mathrm{~B} 2 & \mathrm{~A} 1 & \mathrm{~B} 2 & \mathrm{~A} 1\end{array}$

ENERGY COMPONENTS 


$\begin{array}{rlr}\text { WAVEFUNCTION NORMALIZATION } & = & 1.0000000000 \\ \text { ONE ELECTRON ENERGY } & = & -1.2815485671 \\ \text { TWO ELECTRON ENERGY }= & 0.2026393777 \\ \text { NUCLEAR REPULSION ENERGY }= & 0.2102029432 \\ \text { TOTAL ENERGY }= & -0.8687062461 \\ & \\ \text { ELECTRON-ELECTRON POTENTIAL ENERGY }= & 0.2026393777 \\ \text { NUCLEUS-ELECTRON POTENTIAL ENERGY }= & -2.5231354914 \\ \text { NUCLEUS-NUCLEUS POTENTIAL ENERGY }= & 0.2102029432 \\ \text { TOTAL POTENTIAL ENERGY } & = & ---1.1102931704 \\ \text { TOTAL KINETIC ENERGY } & = & 1.2415869243 \\ \text { VIRIAL RATIO }(\mathrm{V} / \mathrm{T}) & = & 1.6996741260\end{array}$

COEFFICIENT/ OCCUPIED ACTIVE SPIN ORBITALS

$0.7070999672 \mathrm{E}+00 \quad 2 \quad-1$

$\begin{array}{llll}-0.7070999672 E+00 & 1 & -2\end{array}$

Atomic Contributions for neq: 1

Atom number : 1

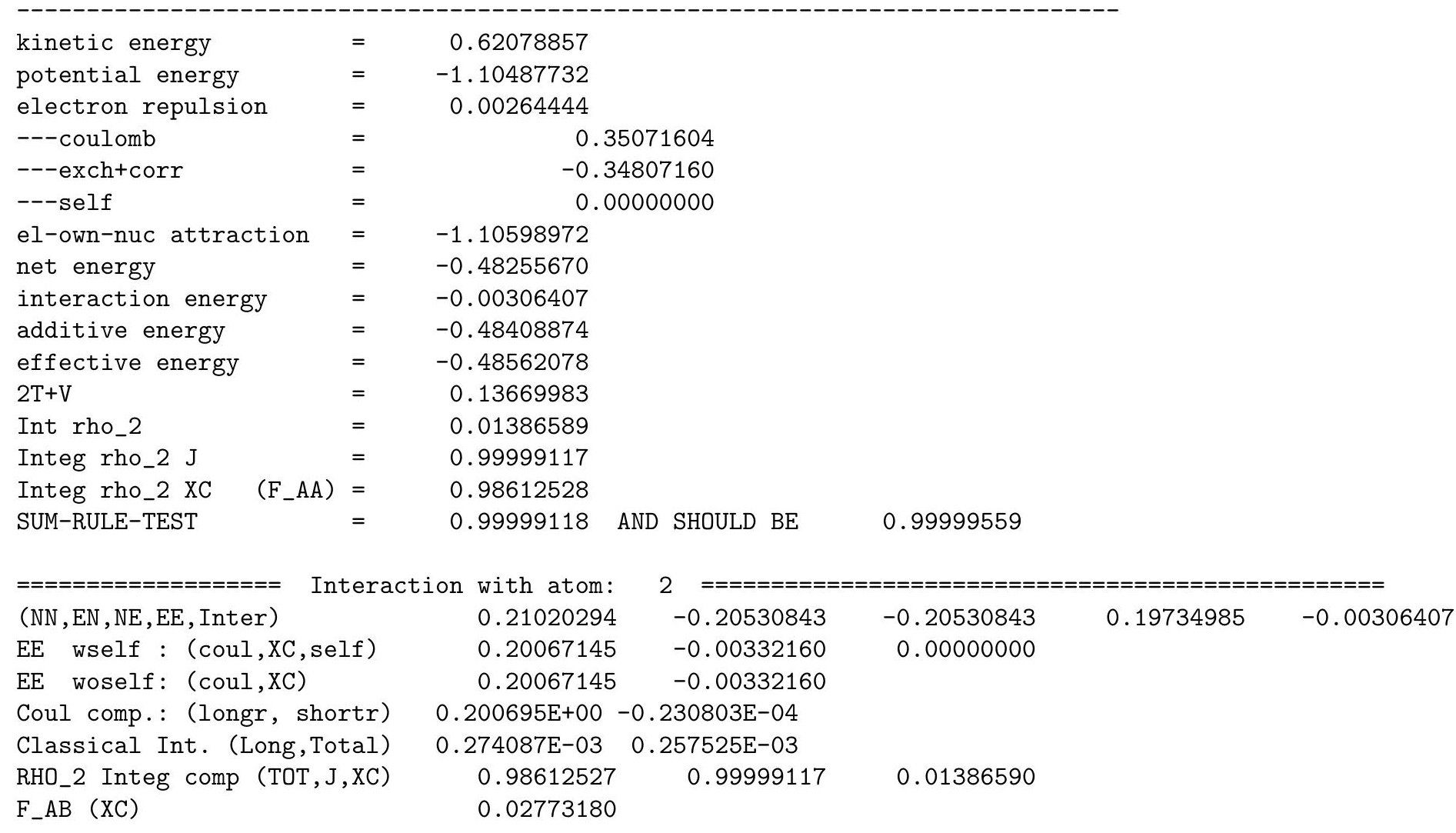

\subsection{ECP H-He-H BS}

EIGENVECTORS

$\begin{array}{ccccc}1 & 2 & 3 & 4 & 5 \\ -0.2499 & -0.0022 & 0.4281 & 0.4345 & 1.3896 \\ \mathrm{~A} 1 & \mathrm{~B} 2 & \mathrm{~A} 1 & \mathrm{~B} 2 & \mathrm{~A} 1\end{array}$


NATURAL ORBITALS IN ATOMIC ORBITAL BASIS

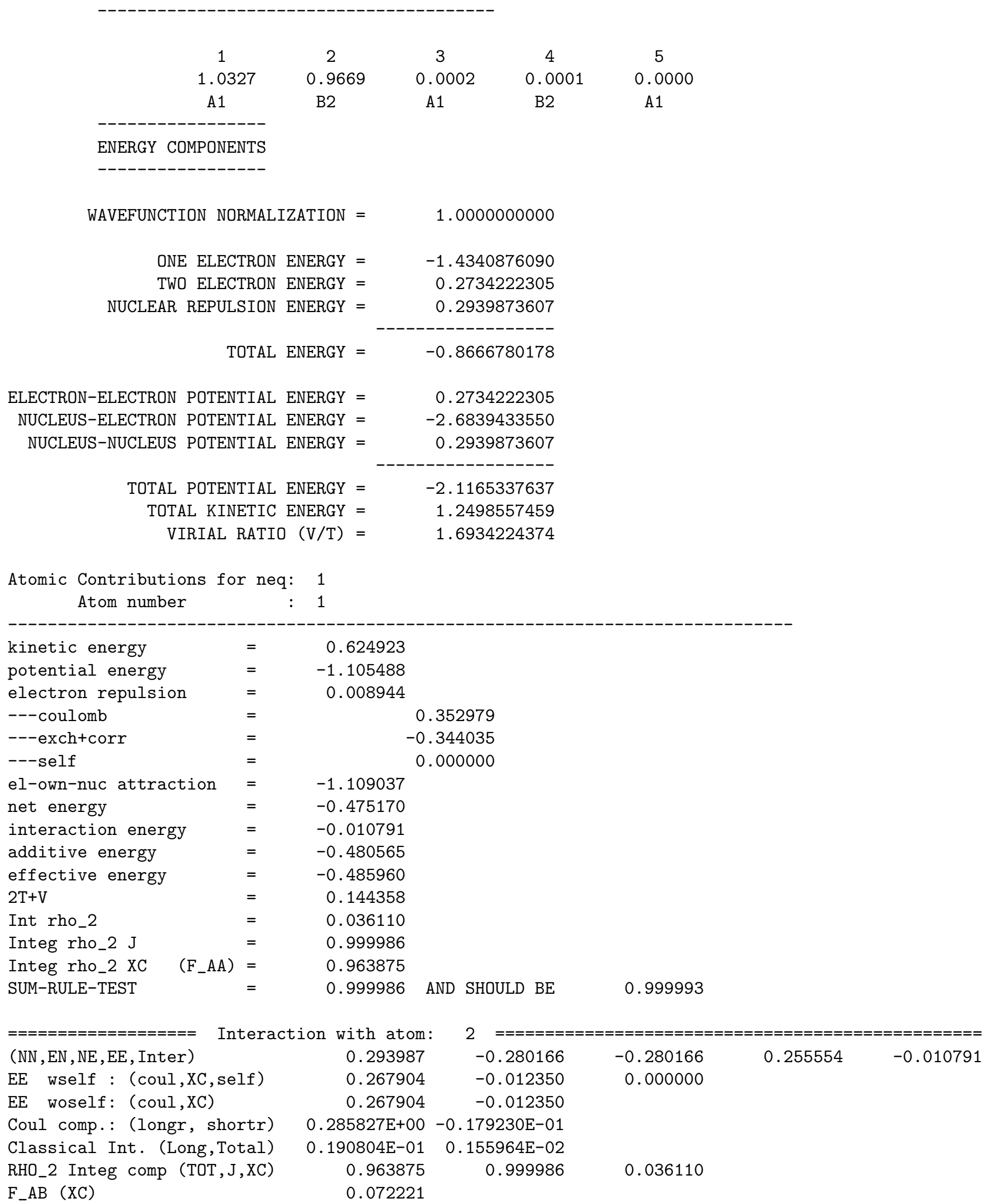

\subsection{ECP Н-He-H ВТ}




$\begin{array}{ccccc}1 & 2 & 3 & 4 & 5 \\ -0.4609 & -0.4336 & 0.3670 & 0.3947 & 1.3248 \\ \mathrm{~A} 1 & \mathrm{~B} 2 & \mathrm{~B} 2 & \mathrm{~A} 1 & \mathrm{~A} 1\end{array}$

NATURAL ORBITALS IN ATOMIC ORBITAL BASIS

$\begin{array}{ccccc}1 & 2 & 3 & 4 & 5 \\ 0.9997 & 0.9997 & 0.0002 & 0.0002 & 0.0000 \\ \text { B2 } & \text { A1 } & \text { A1 } & \text { B2 } & \text { B2 }\end{array}$

ENERGY COMPONENTS

WAVEFUNCTION NORMALIZATION $=$

1.0000000000

ONE ELECTRON ENERGY =

$-1.4342355455$

TWO ELECTRON ENERGY $=0.2690934408$

NUCLEAR REPULSION ENERGY $=$

0.2939873607

TOTAL ENERGY $=\quad-0.8711547440$

$\begin{array}{rr}\text { ELECTRON-ELECTRON POTENTIAL ENERGY }= & 0.2690934408 \\ \text { NUCLEUS-ELECTRON POTENTIAL ENERGY }= & -2.6702806335 \\ \text { NUCLEUS-NUCLEUS POTENTIAL ENERGY }= & 0.2939873607 \\ & ---1 \\ \text { TOTAL POTENTIAL ENERGY }= & -2.1071998319 \\ \text { TOTAL KINETIC ENERGY }= & 1.2360450880 \\ \text { VIRIAL RATIO }(\mathrm{V} / \mathrm{T})= & 1.7047920439\end{array}$

Atomic Contributions for neq: 1

Atom number

: 1

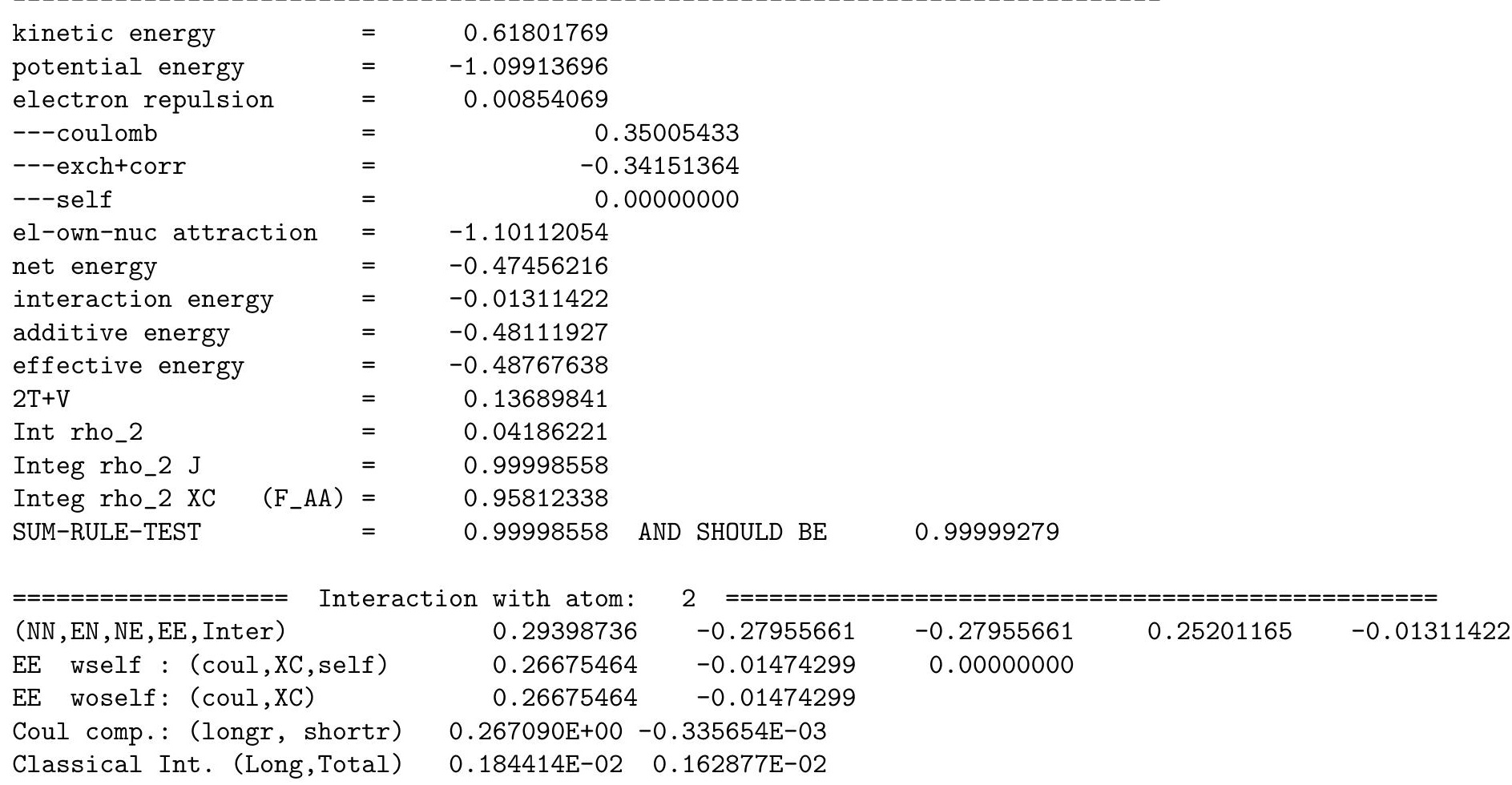




$\begin{array}{llll}\text { RHO_2 Integ comp (TOT, J , XC) } & 0.95812337 & 0.99998558 & 0.04186221 \\ \text { F_AB (XC) } & 0.08372441 & & \end{array}$

\subsection{H-Be-H ${ }^{2+}$ LS}

Geometries and numbering as in the H-He-H calculations.

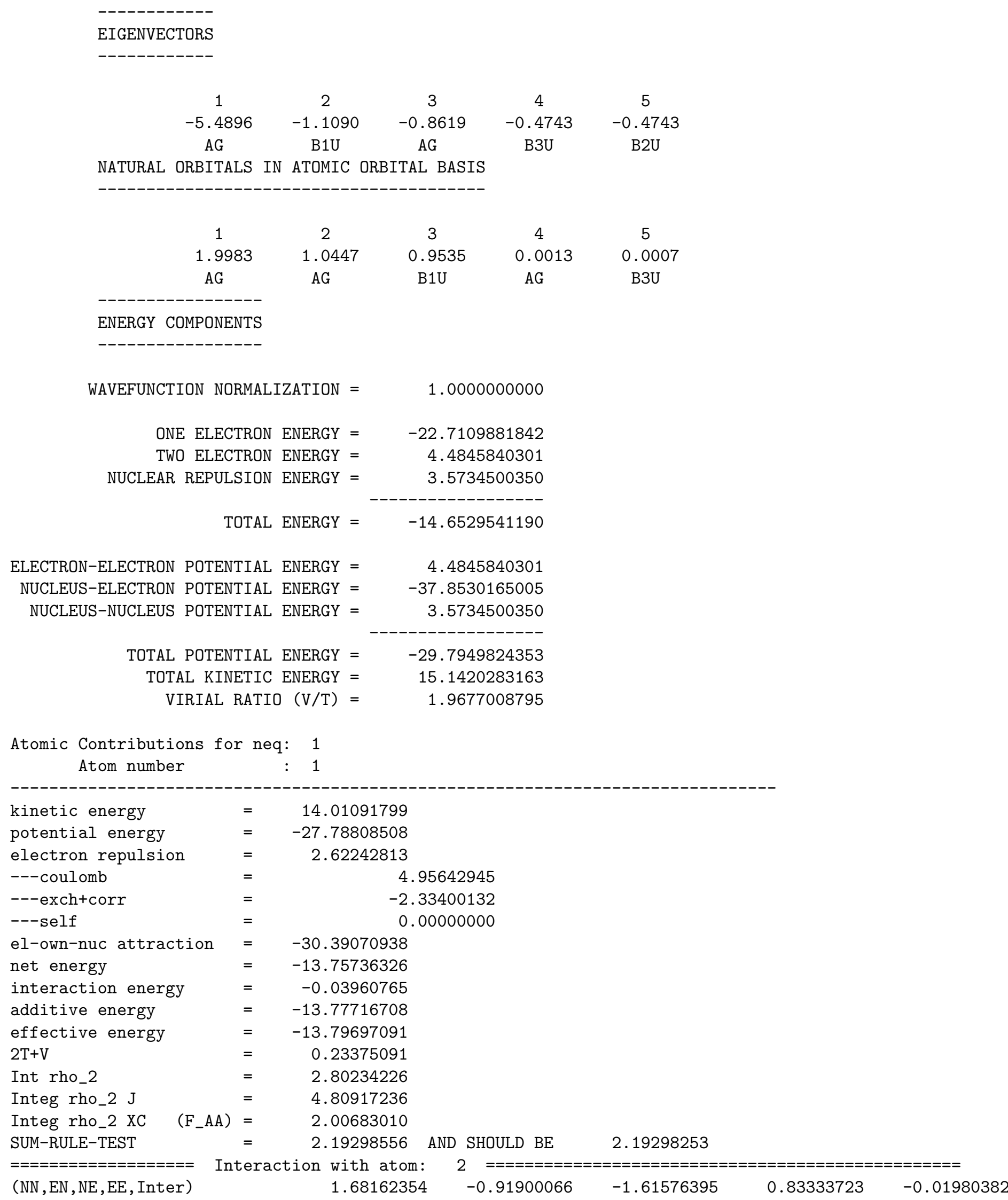


EE wself : (coul,XC, self)

EE woself: (coul, XC)

Coul comp.: (longr, shortr)

Classical Int. (Long,Total)

RHO_2 Integ comp (TOT, J,XC)

F_AB (XC)

$\begin{array}{ccc}0.88318239 & -0.04984516 & 0.00000000 \\ 0.88318239 & -0.04984516 & \\ 0.581699 \mathrm{E}+00 & 0.301483 \mathrm{E}+00 & \\ -0.288440 \mathrm{E}+00 & 0.300413 \mathrm{E}-01 & \\ 1.88834987 & 1.98142760 & 0.09307773 \\ 0.18615546 & & \end{array}$

$==================$ Interaction with atom:

3

EE wself : (coul,XC, self) 0.88318239

0.88318239

$-0.91900066$

$-1.61576395$

0.83333723

$-0.01980382$

EE woself: (coul, XC)

$0.581699 \mathrm{E}+00$

$-0.288440 \mathrm{E}+00$

$-0.04984516$

0.00000000

$-0.04984516$

$0.301483 E+00$

$0.300413 \mathrm{E}-01$

1.88834987
0.18615546

1.98142760

0.09307773

RHO_2 Int
F_AB (XC)

Atomic Contributions for neq: 2

Atom number

: 2

$\begin{array}{llr}\text { kinetic energy } & = & 0.56556243 \\ \text { potential energy } & = & -1.00342083 \\ \text { electron repulsion } & = & 0.00381273 \\ \text {---coulomb } & = & 0.30132833 \\ \text {---exch+corr } & = & -0.29751560 \\ \text {---self } & = & 0.00000000\end{array}$

el-own-nuc attraction $=-0.99651542$

net energy $=-0.42714026$

interaction energy $=-0.02143628$

additive energy $=-0.43785840$

effective energy $\quad=\quad-0.44857654$

$2 \mathrm{~T}+\mathrm{V}$

Int rho_2

Integ rho_2 J

Integ rho_2 XC

SUM-RULE-TEST

$\begin{array}{rll} & = & 0.12770403 \\ & = & 0.01472889 \\ & = & 0.81636819 \\ \left(F_{-} A A\right) & = & 0.80163931 \\ & = & 0.90355168\end{array}$

AND SHOULD BE

0.90353096

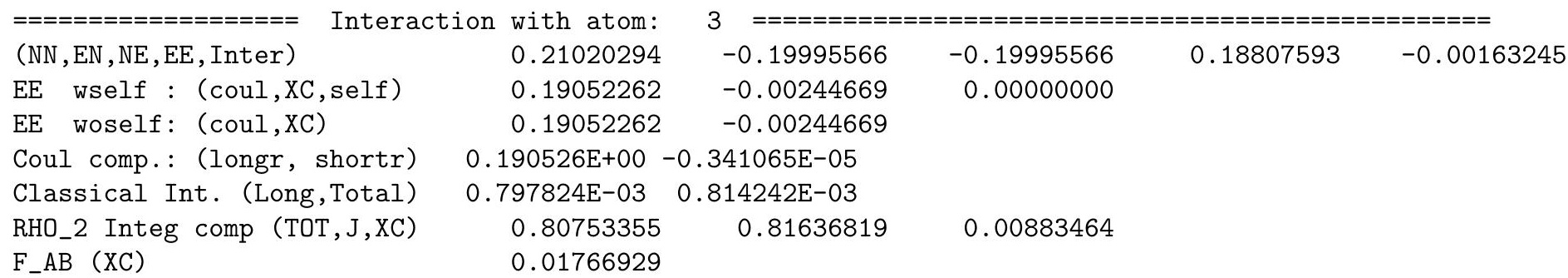

COEFFICIENT/ OCCUPIED ACTIVE SPIN ORBITALS

$\begin{array}{rllll}0.7224233093 \mathrm{E}+00 & 1 & 2 & -1 & -2 \\ -0.6901499037 \mathrm{E}+00 & 1 & 3 & -1 & -3 \\ -0.1730925646 \mathrm{E}-01 & 2 & 4 & -2 & -4 \\ -0.1729894884 \mathrm{E}-01 & 1 & 6 & -1 & -6 \\ -0.1729894884 \mathrm{E}-01 & 1 & 5 & -1 & -5 \\ 0.1660246313 \mathrm{E}-01 & 3 & 4 & -3 & -4 \\ 0.1297446546 \mathrm{E}-01 & 1 & 7 & -1 & -7\end{array}$

\# M-BASINS SPINLESS ELECTRON DISTRIBUTION FUNCTION

\begin{tabular}{|c|c|}
\hline \# NUMBER OF GROUPS & $=$ \\
\hline \# TOTAL NUMBER OF PROBABILITIES & 15 \\
\hline
\end{tabular}




\begin{tabular}{|c|c|c|c|c|}
\hline$\#$ & 0.7955322188862616 & 2 & 1 & 1 \\
\hline \# & 0.0903557769930355 & 3 & 0 & 1 \\
\hline$\#$ & 0.0903557769930355 & 3 & 1 & 0 \\
\hline \# & 0.0091551200670438 & 4 & 0 & 0 \\
\hline \# & 0.0043139690496871 & 2 & 0 & 2 \\
\hline \# & 0.0043139690496871 & 2 & 2 & 0 \\
\hline$\#$ & 0.0029850245554108 & 1 & 1 & 2 \\
\hline \# & 0.0029850245554108 & 1 & 2 & 1 \\
\hline$\#$ & 0.0000157444703766 & 1 & 0 & 3 \\
\hline \# & 0.0000157444703766 & 1 & 3 & 0 \\
\hline$\#$ & 0.0000117368815215 & 0 & 2 & 2 \\
\hline \# & 0.0000021636222075 & 0 & 1 & 3 \\
\hline$\#$ & 0.0000021636222075 & 0 & 3 & 1 \\
\hline \# & 0.0000000119755672 & 0 & 0 & 4 \\
\hline$\#$ & 0.0000000119755672 & 0 & 4 & 0 \\
\hline & & & & ---------------------------------- \\
\hline \# & 1.00004 & $<--$ SUM, & 15 & PROBABILITIES > 0.0000000000E+00 \\
\hline \# & 1.0000444571673964 & $<---$ TOTAL & SUM & \\
\hline
\end{tabular}

Average populations and localization indices

$\#\langle\mathrm{n}(\mathrm{1})\rangle=2.1930769942$

$\#\langle\mathrm{n}(2)\rangle \quad=\quad 0.9035504172$

$\#\langle\mathrm{n}(3)\rangle \quad=\quad 0.9035504172$

$\#<\mathrm{n}(2) \mathrm{n}(1)>\quad=\quad 1.8883899520$

$\#<\mathrm{n}(3) \mathrm{n}(1)>\quad=\quad 1.8883899520$

$\#\langle\mathrm{n}(3) \mathrm{n}(\mathrm{2})\rangle=0.8075322464$

$\#<\mathrm{n}(3) \mathrm{n}(2) \mathrm{n}(1)>=1.6030045360$

\# delta_( 111$) \quad=\quad 2.0071356240 \%$ Localization $=91.5214$

$\#$ delta_( 2 2 $\quad=0.8016743032 \%$ Localization $=88.7249$

$\#$ delta_ $\left(\begin{array}{ll}3 & 3\end{array}\right) \quad=\quad 0.8016743032 \%$ Localization $=88.7249$

Delocalization indices, Eq. (28) J. Chem. Phys. 126, 094102 (2007)

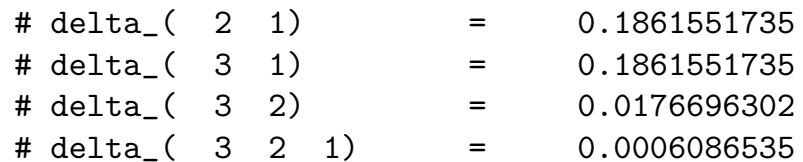

\# Fragment A formed by atoms 1

\# Fragment B formed by atoms 23

\#

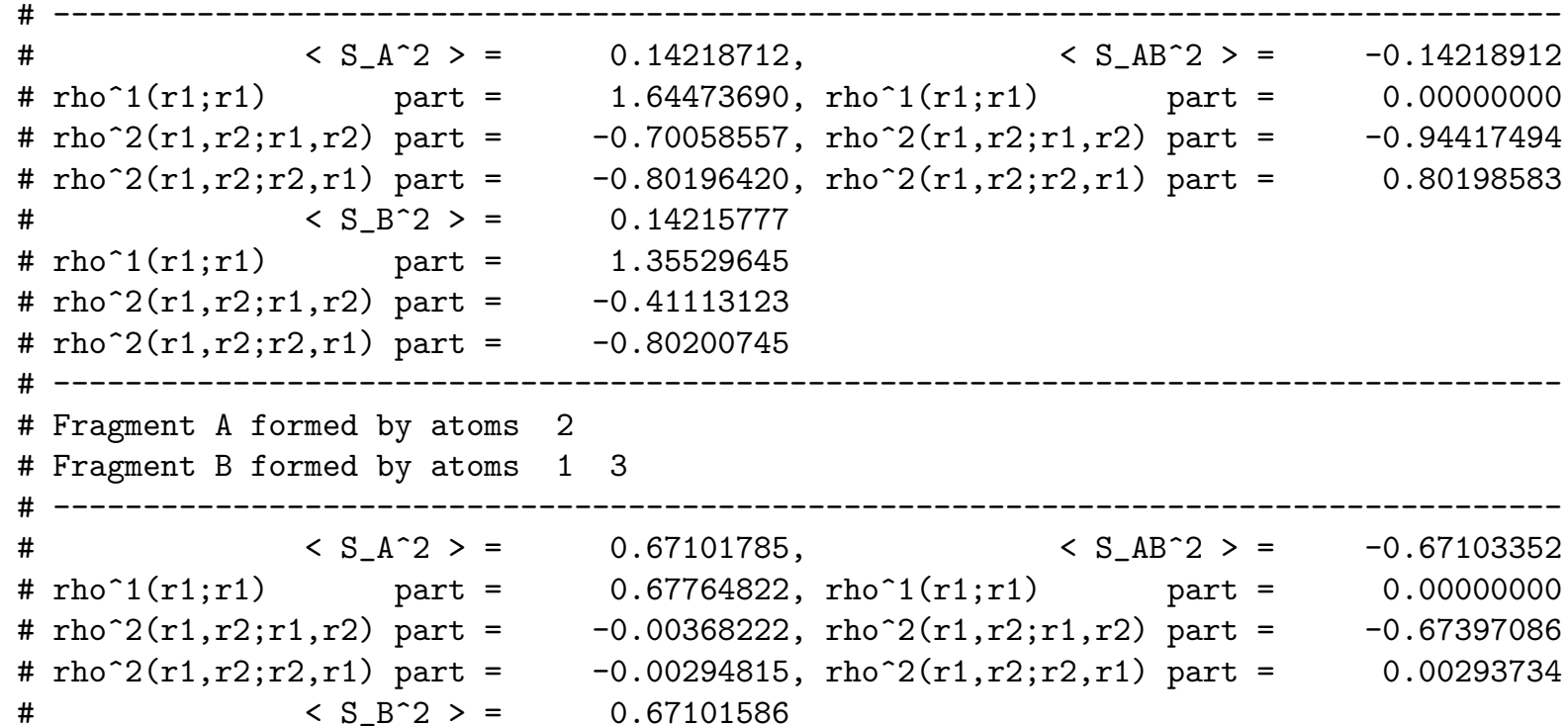


\# rho^1(r1;r1) $\quad$ part $=\quad 2.32238512$

\# rho^2(r1,r2;r1,r2) part $=\quad-1.64844274$

\# rho^2(r1,r2;r2,r1) part $=\quad-0.00292653$

\section{$4.10 \mathrm{H}^{-B e-H^{2+}} \mathrm{LT}$}

Geometries as in the $\mathrm{H}-\mathrm{He}-\mathrm{H}$ calculations.

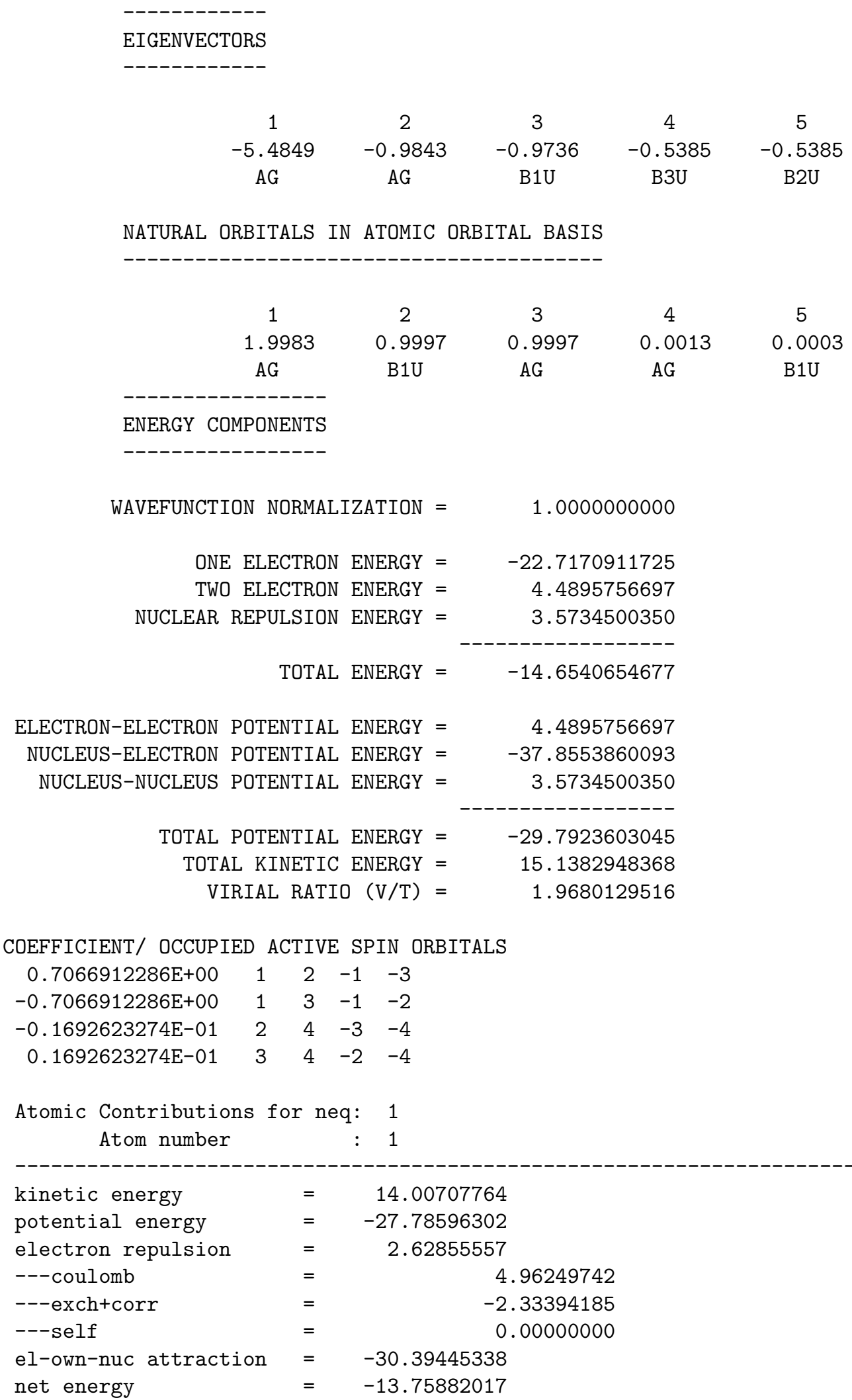




$\begin{array}{llr}\text { interaction energy } & = & -0.04013043 \\ \text { additive energy } & = & -13.77888539 \\ \text { effective energy } & = & -13.79895060 \\ \text { 2T+V } & = & 0.22819225 \\ \text { Int rho_2 } & = & 2.81470614 \\ \text { Integ rho_2 J } & 4.82213729 \\ \text { Integ rho_2 XC (F_AA) } & = & 2.00743115 \\ \text { SUM-RULE-TEST } & = & 2.19595438\end{array}$

AND SHOULD BE

2.19593654

\begin{tabular}{|c|c|c|c|c|c|}
\hline $\begin{array}{l}=================\text { lnter } \\
(\mathrm{NN}, \mathrm{EN}, \mathrm{NE}, \mathrm{EE}, \text { Inter })\end{array}$ & $\begin{array}{r}\text { on with atom: } \\
1.68162354\end{array}$ & -0.92055270 & -1.61424579 & 0.83310973 & -0.020065 \\
\hline EE wself : (coul, XC, self) & 0.88382587 & -0.05071614 & 0.00000000 & & \\
\hline EE woself: (coul, XC) & 0.88382587 & -0.05071614 & & & \\
\hline Coul comp.: (longr, shortr) & $0.653805 \mathrm{E}+00$ & $0.230021 \mathrm{E}+00$ & & & \\
\hline Classical Int. (Long,Total) & $-0.217374 \mathrm{E}+00$ & $0.306509 \mathrm{E}-01$ & & & \\
\hline RHO_2 Integ comp (TOT, J,XC) & 1.88666867 & 1.98093029 & 0.09426162 & & \\
\hline$F_{-} A B(X C)$ & 0.18852323 & & & & \\
\hline
\end{tabular}

\begin{tabular}{|c|c|c|c|c|c|}
\hline$===============$ & ion with atom: & $=========$ & $============$ & $==========$ & $======$ \\
\hline $\begin{array}{l}==-=-=-==-==-==-= \\
(\mathrm{NN}, \mathrm{EN}, \mathrm{NE}, \mathrm{EE}, \text { Inter })\end{array}$ & 1.68162354 & -0.92055270 & -1.61424579 & 0.83310973 & -0.02006521 \\
\hline EE wself : (coul, XC,self) & 0.88382587 & -0.05071614 & 0.00000000 & & \\
\hline EE woself: (coul, XC) & 0.88382587 & -0.05071614 & & & \\
\hline Coul comp.: (longr, shortr) & $0.653805 \mathrm{E}+00$ & $0.230021 \mathrm{E}+00$ & & & \\
\hline Classical Int. (Long,Total) & $-0.217374 \mathrm{E}+00$ & $0.306509 \mathrm{E}-01$ & & & \\
\hline RHO_2 Integ comp (TOT, J,XC) & 1.88666867 & 1.98093029 & 0.09426162 & & \\
\hline$F_{-} A B(X C)$ & 0.18852323 & & & & \\
\hline
\end{tabular}

Atomic Contributions for neq: 2

Atom number : 2

$\begin{array}{llr}\text { kinetic energy } & = & 0.56565557 \\ \text { potential energy } & = & -1.00323696 \\ \text { electron repulsion } & = & 0.00376560 \\ \text {---coulomb } & = & 0.30100904 \\ \text {---exch+corr } & = & 0.00000000 \\ \text {---self } & = & -0.99622292 \\ \text { el-own-nuc attraction } & = & -0.42680174 \\ \text { net energy } & = & -0.02155930 \\ \text { interaction energy } & = & -0.43758139 \\ \text { additive energy } & = & -0.44836104 \\ \text { effective energy } & = & 0.12807418 \\ \text { 2T+V } & = & 0.01486399 \\ \text { Int rho_2 } & = & 0.81376464 \\ \text { Integ rho_2 J } & = & 0.79890064 \\ \text { Integ rho_2 XC (F_AA) } & = & 0.90213743 \quad \text { AND SHOULD BE } \\ \text { SUM-RULE-TEST } & = & \end{array}$

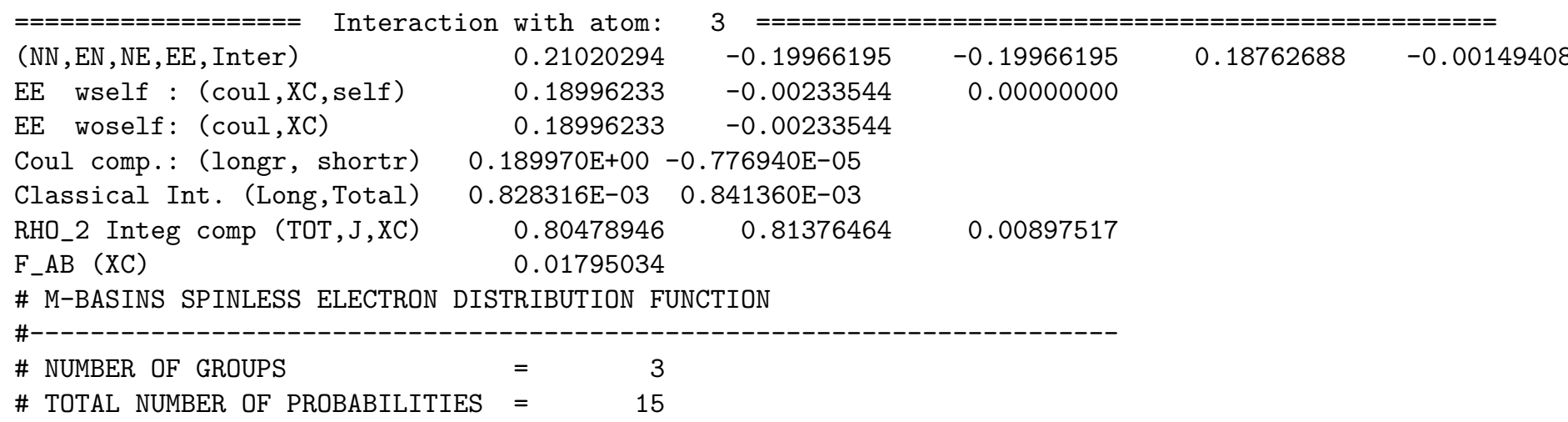




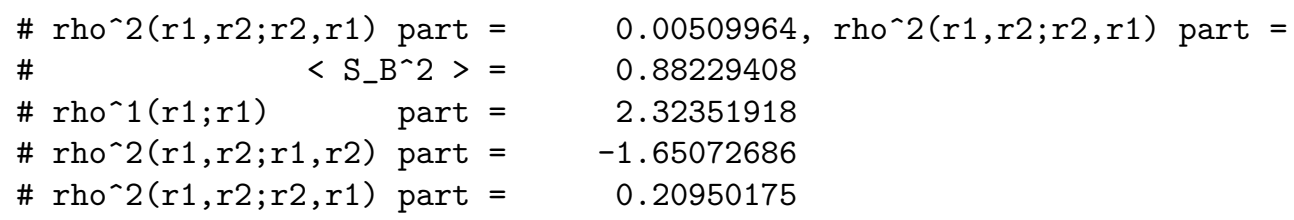

\section{$4.11{\mathrm{H}-\mathrm{Be}-\mathrm{H}^{2+} \mathrm{BS}}^{2}$}

Geometries as in the $\mathrm{H}-\mathrm{He}-\mathrm{H}$ calculations.

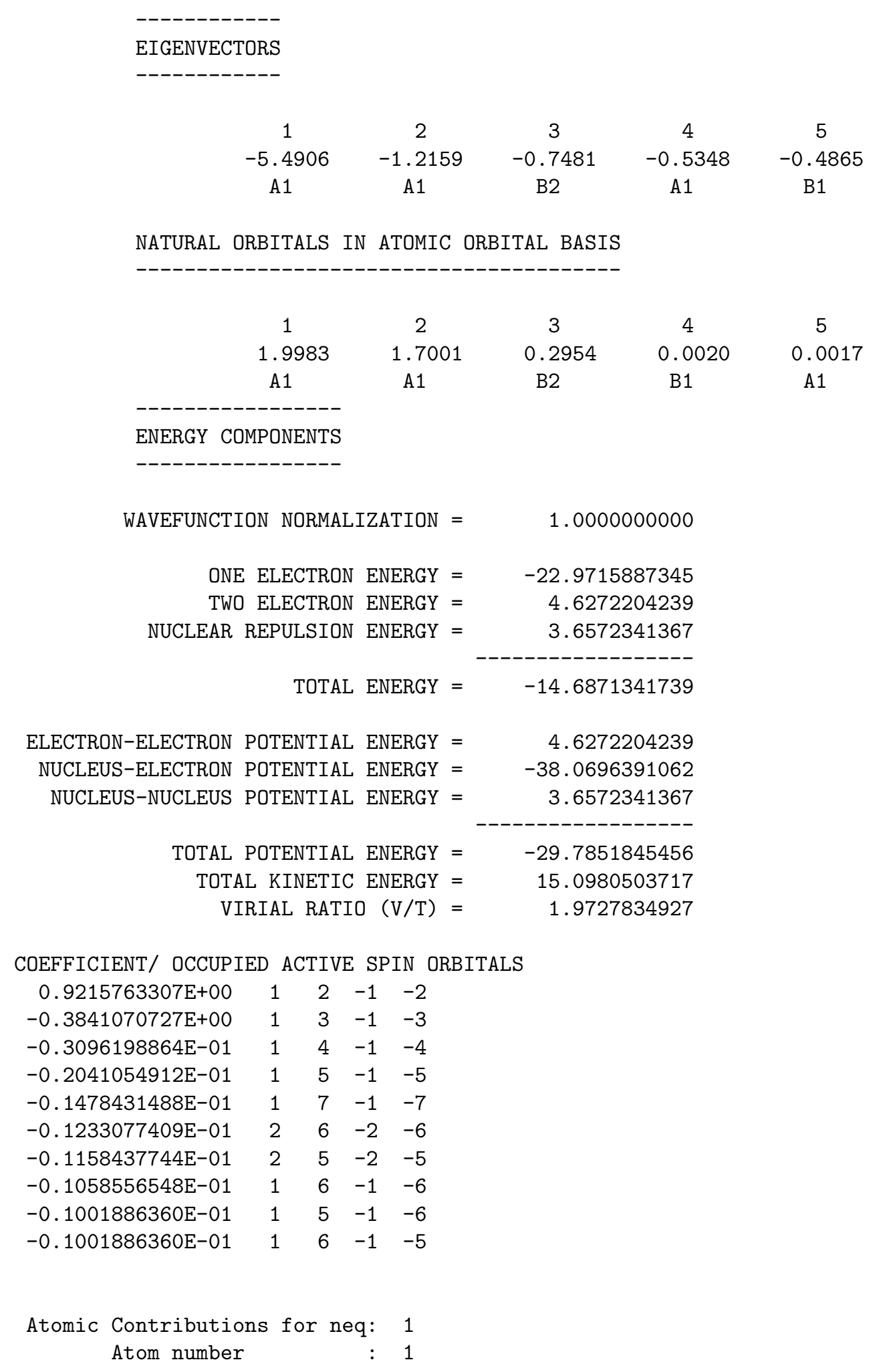




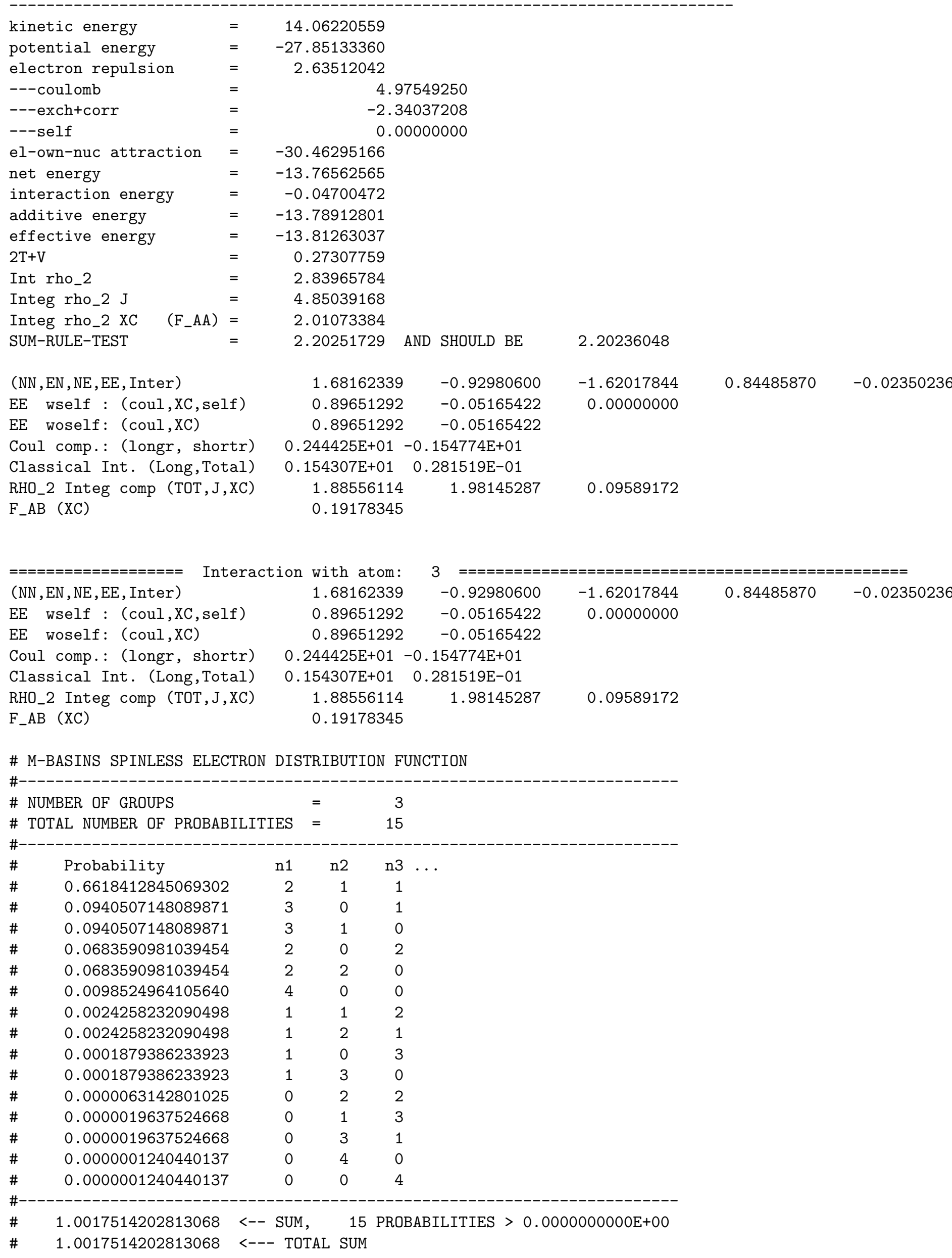


\#-

Average populations and localization indices

$\#\langle\mathrm{n}(1)\rangle \quad=2.2060607596$

$\#\langle\mathrm{n}(2)\rangle \quad=0.9004724608$

$\#\langle\mathrm{n}(3)\rangle \quad=\quad 0.9004724608$

$\#<\mathrm{n}(2) \mathrm{n}(1)>\quad=\quad 1.8871123914$

$\#\langle\mathrm{n}(3) \mathrm{n}(1)\rangle=1.8871123914$

$\#\langle\mathrm{n}(3) \mathrm{n}(\mathrm{2})\rangle=0.6715816170$

$\left.\#<\mathrm{n}(3) \mathrm{n}\left(\begin{array}{l}2 \\ \text { 3 }\end{array}\right) \mathrm{n}(1)\right\rangle=1.3333858619$

\# delta_( 11 1 $) \quad=\quad 2.0227465789 \%$ Localization $=91.6904$

$\#$ delta_( 2 2 2$) \quad=0.6681272786 \%$ Localization $=74.1974$

$\#$ delta_( 3 3 $) \quad=0.6681272786 \%$ Localization $=74.1974$

Delocalization indices, Eq. (28) J. Chem. Phys. 126, 094102 (2007)

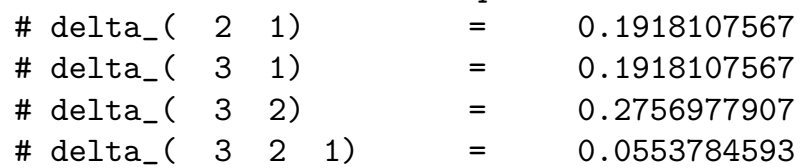

\# Fragment A formed by atoms 1

\# Fragment B formed by atoms 23

\#

\# rho^1(r1;r1) part $=1.65177036, r h 0^{\wedge 1}(r 1 ; r 1) \quad$ part $=\quad 0.00000000$

\# rho^2(r1,r2;r1,r2) part $=\quad-0.70991446$, rho^2(r1,r2;r1,r2) part $=\quad-0.94278057$

\# rho^2(r1,r2;r2,r1) part $=-0.79507999$, rho^2(r1,r2;r2,r1) part $=0.79585780$

\# $\quad\left\langle\mathrm{S}_{-} \mathrm{B}^{\wedge} 2>=0.14575543\right.$

\# rho-1(r1;r1) $\quad$ part $=\quad 1.34954261$

\# rho^2(r1,r2;r1,r2) part $=\quad-0.40715073$

\# rho^2(r1,r2;r2,r1) part $=\quad-0.79663644$

\# Fragment A formed by atoms 2

\# Fragment B formed by atoms 13

\# ------

\# $\quad$ S S_A^2 $>=0.57196324, \quad\left\langle\mathrm{~S}_{-} \mathrm{AB}^{\wedge} 2>=\quad-0.57254691\right.$

\# rho-1(r1;r1) part $=0.67477130, r h 0^{-1}(r 1 ; r 1) \quad$ part $=\quad 0.00000000$

\# rho^2(r1,r2;r1,r2) part $=-0.03567993$, rho^2(r1,r2;r1,r2) part $=-0.63928572$

\# rho^2(r1,r2;r2,r1) part $=-0.06712813$, rho^2(r1,r2;r2,r1) part $=0.06673881$

\# $\quad\left\langle\mathrm{S}_{-} \mathrm{B}^{\wedge} 2>=0.57181639\right.$

\# rho^1(r1;r1) part $=\quad 2.32654166$

\# rho^2(r1,r2;r1,r2) part $=\quad-1.68837496$

\# rho^2(r1,r2;r2,r1) part $=-0.06635032$

\section{$4.12 \mathrm{H}-\mathrm{Be}-\mathrm{H}^{2+} \mathrm{BT}$}

Geometries as in the $\mathrm{H}-\mathrm{He}-\mathrm{H}$ calculations.

EIGENVECTORS

$\begin{array}{ccccc}1 & 2 & 3 & 4 & 5 \\ -5.4983 & -1.0475 & -0.8903 & -0.5589 & -0.5336 \\ \mathrm{~A} 1 & \mathrm{~A} 1 & \mathrm{~B} 2 & \mathrm{~A} 1 & \mathrm{~B} 1\end{array}$


NATURAL ORBITALS IN ATOMIC ORBITAL BASIS

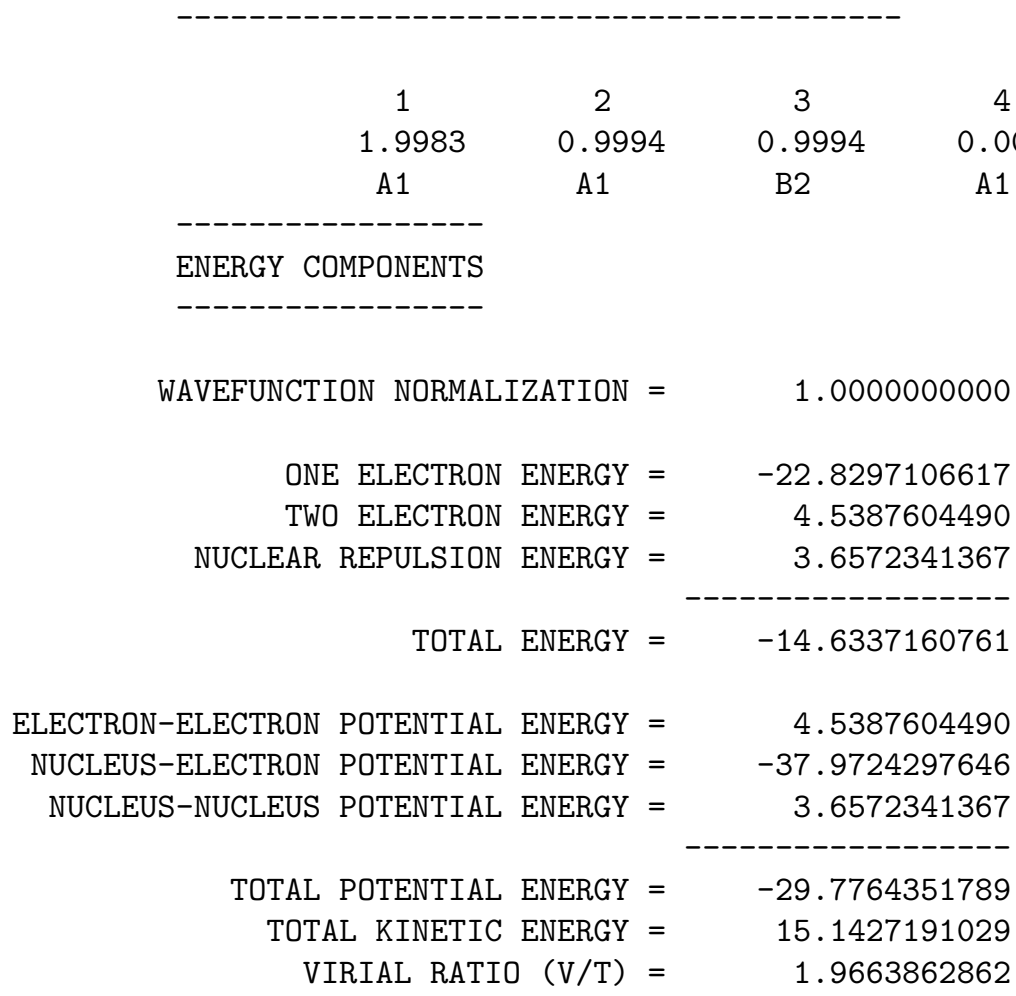

$\begin{array}{ccccc}\text { COEFFICIENT/ OCCUPIED } & \text { ACTIVE SPIN } \\ 0.7065885946 \mathrm{E}+00 & 1 & 2 & -1 & -3 \\ -0.7065885946 \mathrm{E}+00 & 1 & 3 & -1 & -2 \\ -0.1722482824 \mathrm{E}-01 & 2 & 4 & -3 & -4 \\ 0.1722482824 \mathrm{E}-01 & 3 & 4 & -2 & -4\end{array}$

Atomic Contributions for neq: 1 Atom number

: 1

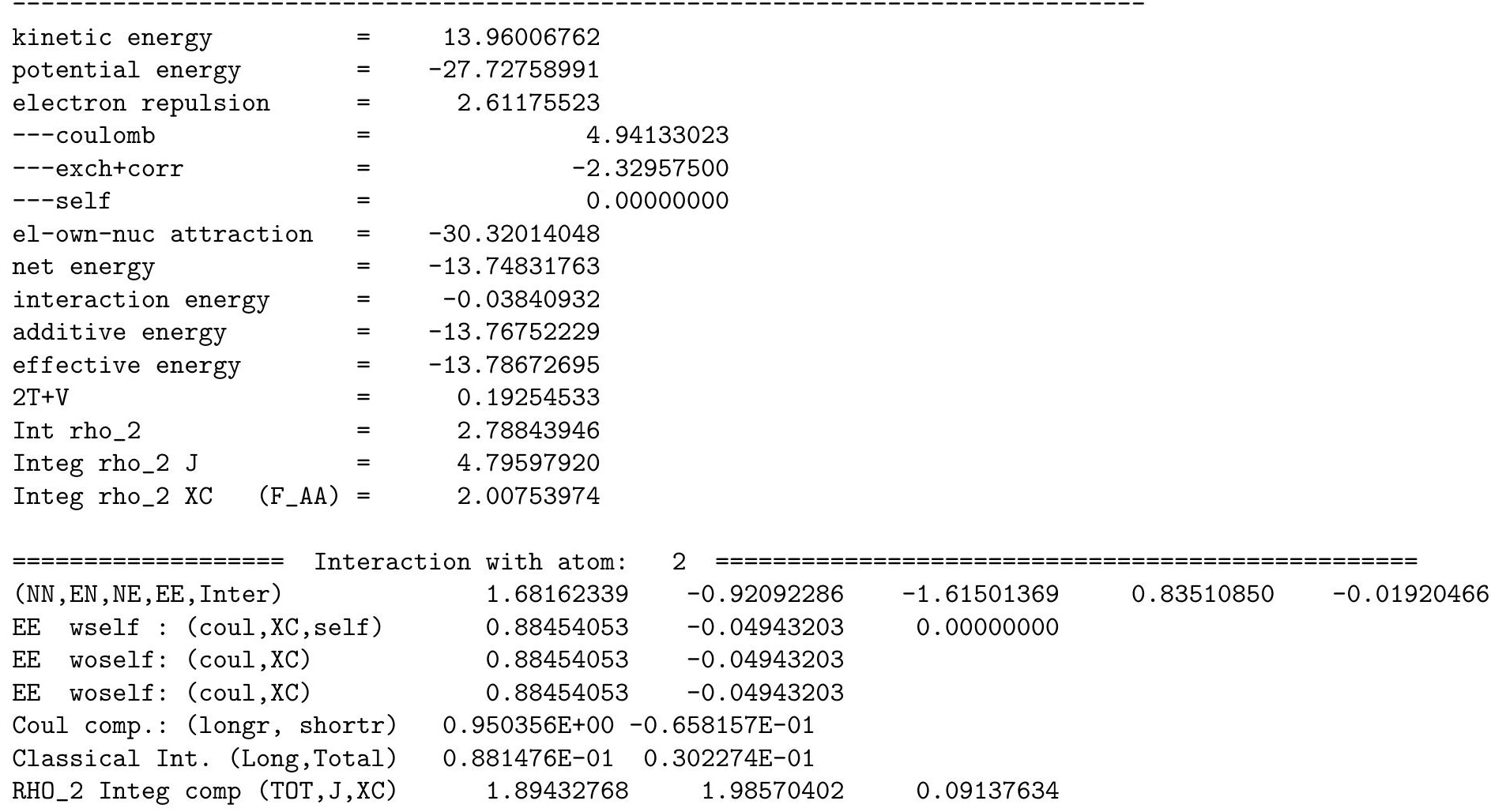




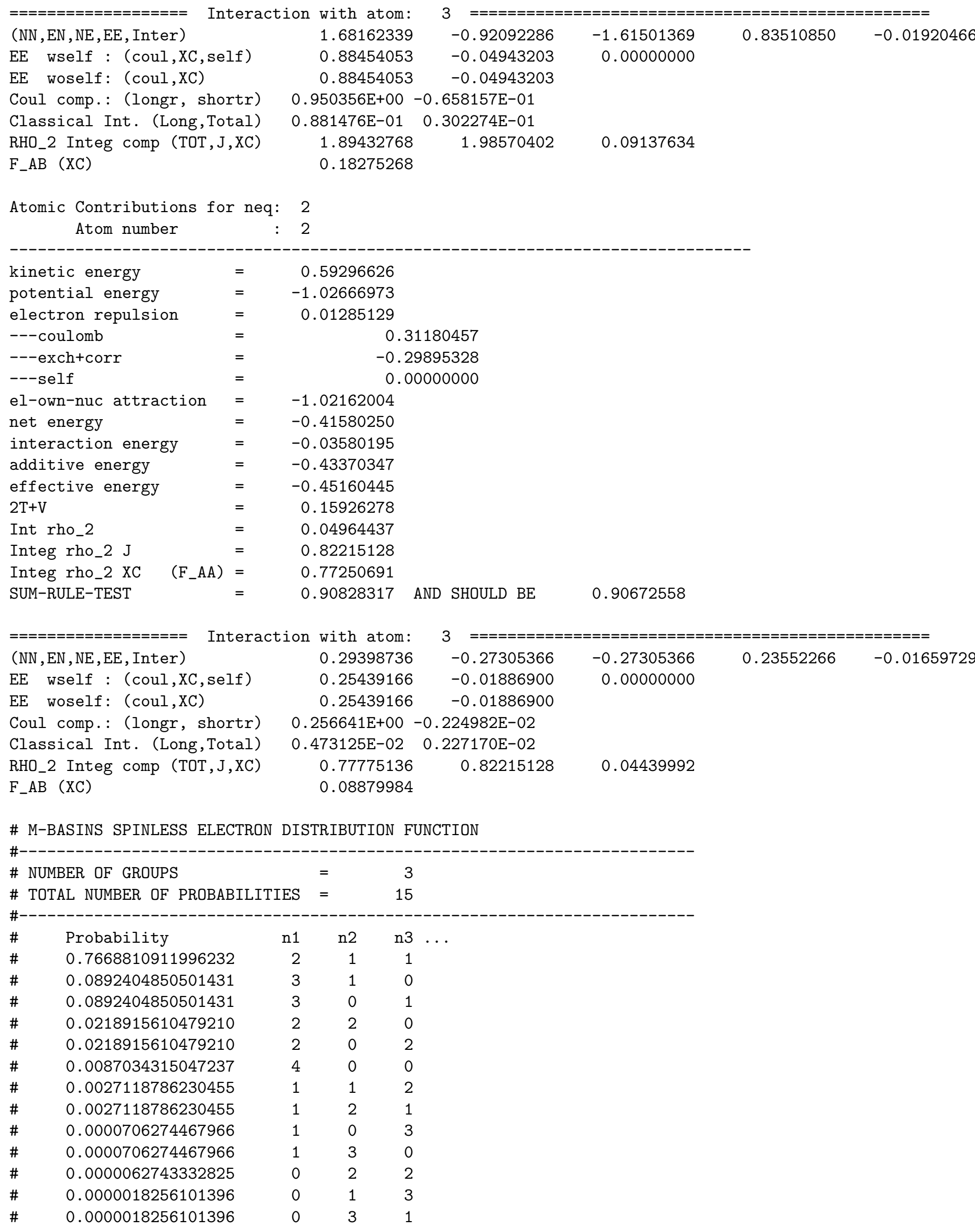




$\begin{array}{lcccc}\# & 0.0000000444991800 & 0 & 0 & 4 \\ \# & 0.0000000444991800 & 0 & 4 & 0 \\ \#---1.0034236415920808 & <-- & \text { SUM, } & 15 \text { PROBABILITIES > 0.0000000000E+00 } \\ \# & 1.0034236415920808 & <-- & \text { TOTAL SUM }\end{array}$

Average populations and localization indices

\begin{tabular}{|c|c|c|c|c|c|c|c|}
\hline & $\langle\mathrm{n}(1)\rangle$ & & & $=$ & 2.1971500751 & & \\
\hline \# & $\langle\mathrm{n}(2)\rangle$ & & & $=$ & 0.9082722457 & & \\
\hline \# & $<\mathrm{n}($ & & & $=$ & 0.9082722457 & & \\
\hline \# & $<\mathrm{n}($ & $\mathrm{n}($ & 1) $>$ & $=$ & 1.8973974000 & & \\
\hline \# & $<\mathrm{n}($ & $\mathrm{n}($ & 1) $>$ & $=$ & 1.8973974000 & & \\
\hline \# & $<\mathrm{n}($ & $\mathrm{n}($ & 2) $>$ & $=$ & 0.7777646567 & & \\
\hline$\#$ & $<\mathrm{n}($ & $\mathrm{n}($ & 2) $n(1)>$ & $=$ & 1.5446096969 & & \\
\hline \# & delta_( & 1 & 1) & $=$ & 2.0308130270 & $\%$ Localization = & $=92.4294$ \\
\hline$\#$ & delta_( & 2 & 2) & $=$ & 0.7753037919 & $\%$ Localization = & $=85.3603$ \\
\hline \# & delta_( & 3 & 3) & $=$ & 0.7753037919 & $\%$ Localization = & $=85.3603$ \\
\hline
\end{tabular}

Delocalization indices, Eq. (28) J. Chem. Phys. 126, 094102 (2007)

\begin{tabular}{|c|c|}
\hline \# delta_ & 2 \\
\hline \# delta_ & 3 \\
\hline \# delta_ & 3 \\
\hline
\end{tabular}

\# delta $\left(\begin{array}{lll}3 & 2 & 1\end{array}\right)=0.0158936385$

\# Fragment A formed by atoms 1

\# Fragment B formed by atoms 23

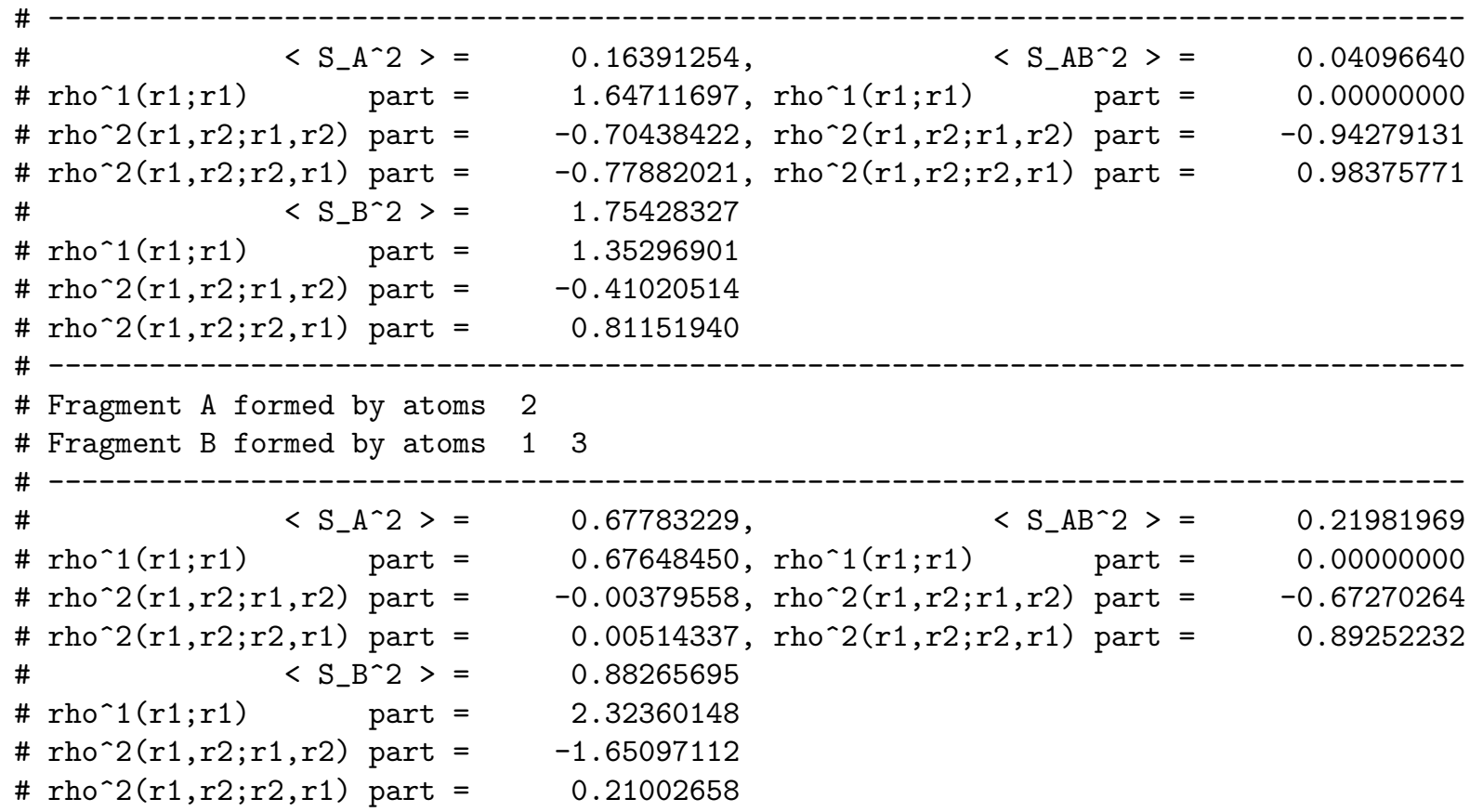

\section{$4.13 \mathrm{H}-\mathrm{He}-\mathrm{H}^{2+}$ LS}

Geometries as in the $\mathrm{H}-\mathrm{He}-\mathrm{H}$ calculations. 


$\begin{array}{ccccc}1 & 2 & 3 & 4 & 5 \\ -1.7745 & -0.7108 & -0.6074 & -0.1802 & -0.1331 \\ \text { AG } & \text { B1U } & \text { AG } & \text { AG } & \text { B1U }\end{array}$

NATURAL ORBITALS IN ATOMIC ORBITAL BASIS

\begin{tabular}{ccccc}
\multicolumn{5}{c}{} \\
1 & 2 & 3 & 4 & 5 \\
1.9828 & 0.0094 & 0.0033 & 0.0021 & 0.0021 \\
AG & AG & B1U & B2U & B3U
\end{tabular}

ENERGY COMPONENTS

$\begin{array}{rlr}\text { WAVEFUNCTION NORMALIZATION }= & 1.0000000000 \\ \text { ONE ELECTRON ENERGY }= & -5.5261499415 \\ \text { TWO ELECTRON ENERGY }= & 0.9372315207 \\ \text { NUCLEAR REPULSION ENERGY }= & 1.8918264891 \\ \text { TOTAL ENERGY }= & -2.6970919316\end{array}$

ELECTRON-ELECTRON POTENTIAL ENERGY =

0.9372315207

NUCLEUS-ELECTRON POTENTIAL ENERGY =

$-8.3627712371$

NUCLEUS-NUCLEUS POTENTIAL ENERGY =

1.8918264891

TOTAL POTENTIAL ENERGY =

$-5.5337132273$

TOTAL KINETIC ENERGY =

2.8366212956

VIRIAL RATIO $(\mathrm{V} / \mathrm{T})=$

1.9508114234

COEFFICIENT/ OCCUPIED ACTIVE SPIN ORBITALS

$\begin{array}{rrr}0.9956866879 \mathrm{E}+00 & 1 & -1 \\ -0.6861742770 \mathrm{E}-01 & 2 & -2 \\ -0.4090585122 \mathrm{E}-01 & 3 & -3 \\ -0.3236571173 \mathrm{E}-01 & 5 & -5 \\ -0.3236571173 \mathrm{E}-01 & 4 & -4\end{array}$

Atomic Contributions for neq: 1

Atom number : 1

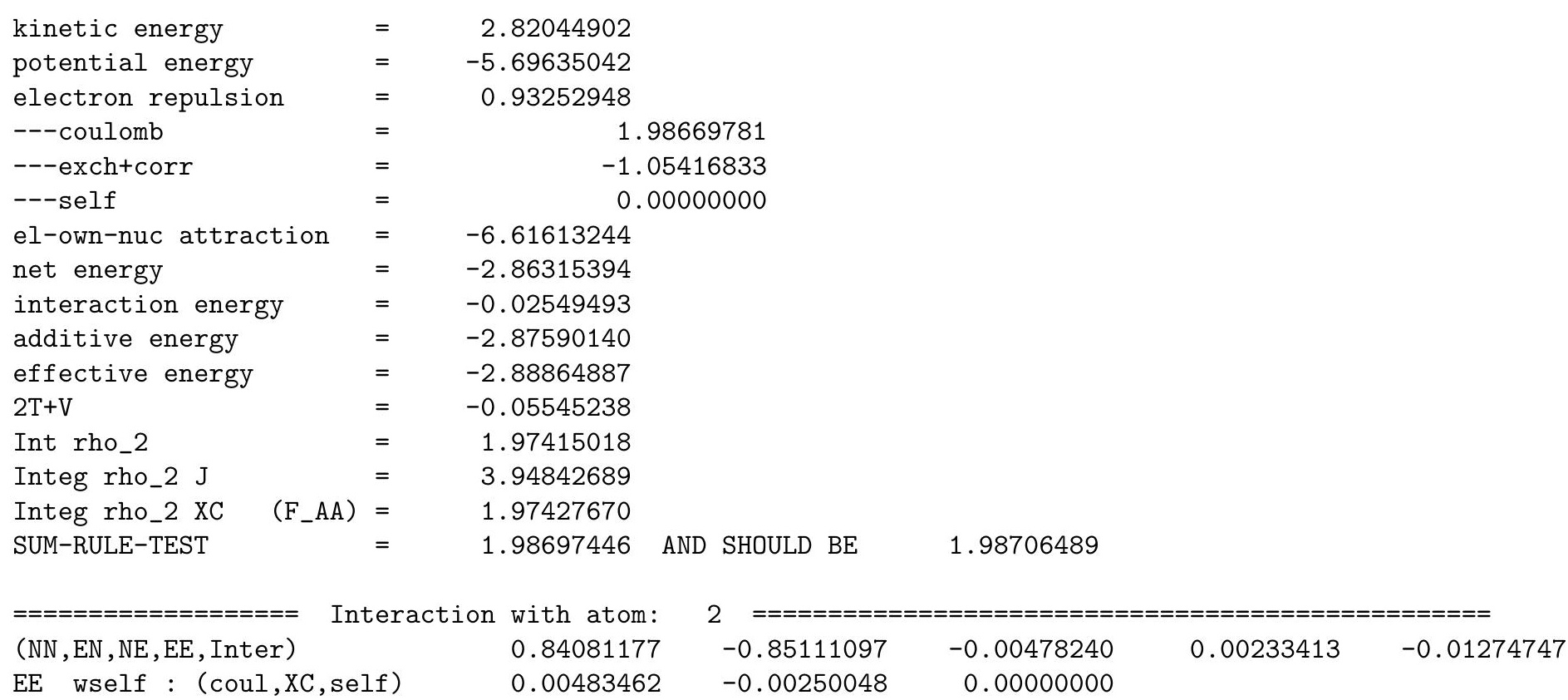


EE woself: (coul, XC)

$0.00483462-0.00250048$

Coul comp.: (longr, shortr)

$0.506580 \mathrm{E}-02-0.231179 \mathrm{E}-03$

Classical Int. (Long, Total)

$-0.143732 \mathrm{E}-02-0.102470 \mathrm{E}-01$

RHO_2 Integ comp (TOT, J,XC)

0.00641177

0.01276065

0.00634888

$F_{-} A B$ (XC)

0.01269775

\begin{tabular}{|c|c|c|c|c|c|}
\hline$===================$ & tion with atom: & $=========3$ & $=============$ & $==========$ & $=======$ \\
\hline (NN, EN, NE, EE, Inter) & 0.84081177 & -0.85111097 & -0.00478240 & 0.00233413 & -0.01274747 \\
\hline EE wself : (coul, XC,self) & 0.00483462 & -0.00250048 & 0.00000000 & & \\
\hline EE woself: (coul, XC) & 0.00483462 & -0.00250048 & & & \\
\hline Coul comp.: (longr, shortr) & $0.506580 \mathrm{E}-02$ & $-0.231179 \mathrm{E}-03$ & & & \\
\hline Classical Int. (Long,Total) & $-0.143732 \mathrm{E}-02$ & $-0.102470 \mathrm{E}-01$ & & & \\
\hline RHO_2 Integ comp (TOT, J,XC) & 0.00641177 & 0.01276065 & 0.00634888 & & \\
\hline$F_{-} A B(X C)$ & 0.01269775 & & & & \\
\hline
\end{tabular}

Atomic Contributions for neq: 2

Atom number : 2

$\begin{array}{llr}\text { kinetic energy } & = & 0.00805362 \\ \text { potential energy } & = & 0.08143451 \\ \text { electron repulsion } & = & 0.00000117 \\ -- \text { coulomb } & = & 0.00003568 \\ -- \text { exch+corr } & = & -0.00003451 \\ -- \text {-self } & = & 0.00000000\end{array}$

el-own-nuc attraction $=-0.01602764$

net energy $=\quad-0.00797284$

interaction energy $=0.19492196$

additive energy $=0.08948813$

effective energy $\quad=\quad 0.18694911$

$2 \mathrm{~T}+\mathrm{V} \quad=0.09754176$

Int rho_2 20.00000148

Integ rho_2 $\mathrm{J}=0.00004124$

Integ rho_2 XC (F_AA) $=0.00003976$

$\begin{array}{llll}\text { SUM-RULE-TEST } & =0.00642137 \text { AND SHOULD BE } & 0.00642186\end{array}$

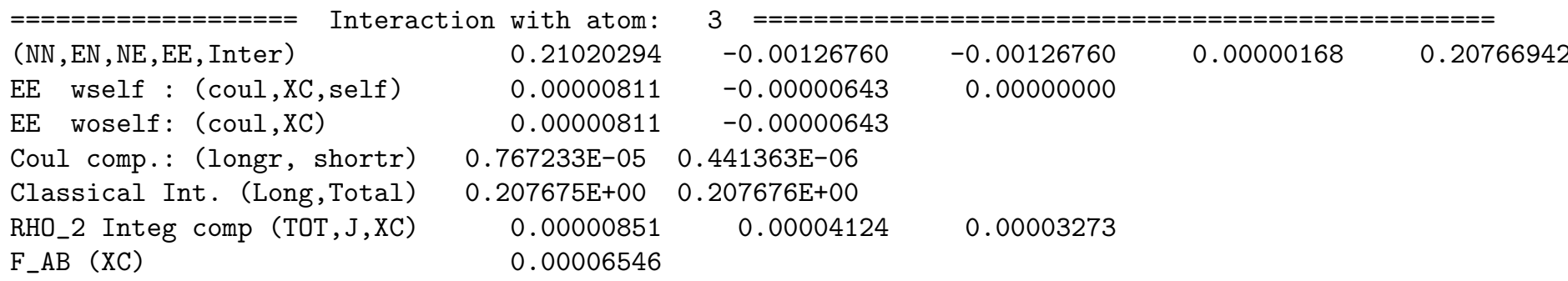

\# M-BASINS SPINLESS ELECTRON DISTRIBUTION FUNCTION

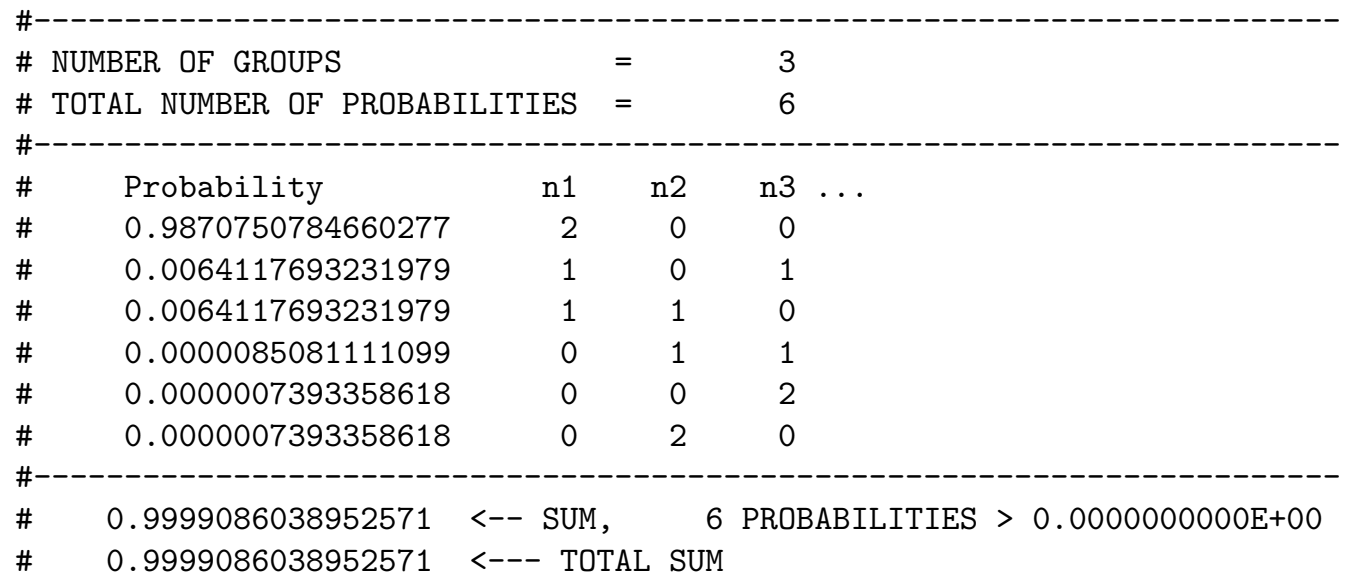




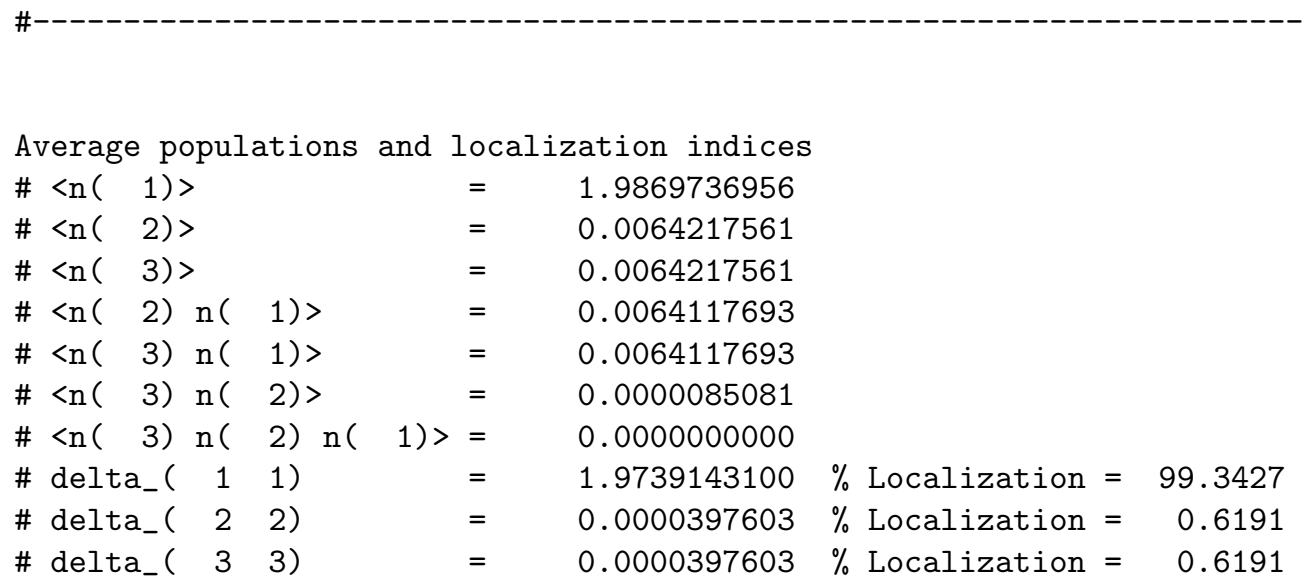

Delocalization indices, Eq. (28) J. Chem. Phys. 126, 094102 (2007)

\begin{tabular}{|c|c|c|}
\hline delta_ & 2 & 0.0126985147 \\
\hline \# delta_ & 3 & 0.0126985147 \\
\hline lelta_ & 3 & $0.00006546 s$ \\
\hline elta_ & 3 & 0.000 \\
\hline
\end{tabular}

\# Fragment A formed by atoms 1

\# Fragment B formed by atoms 23

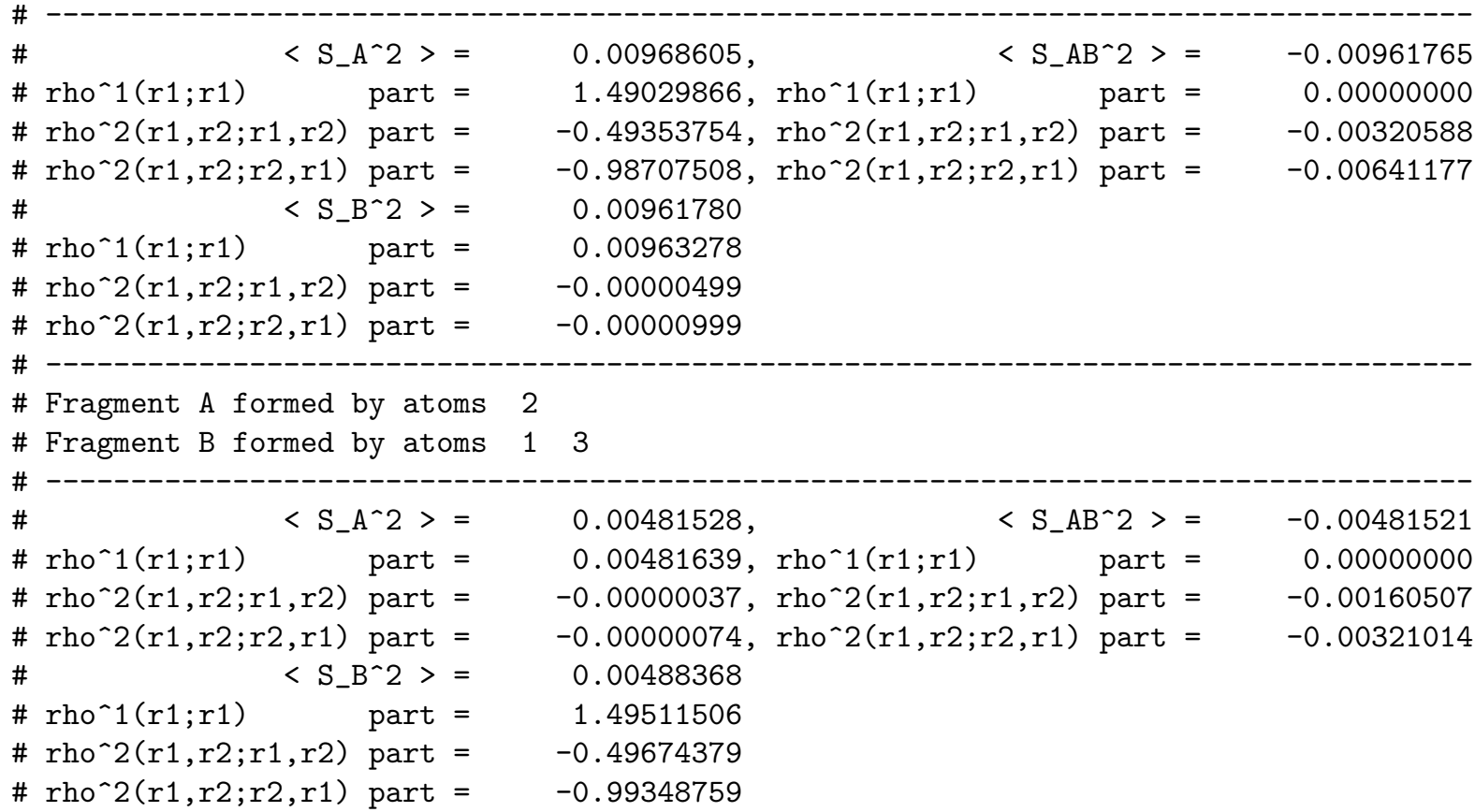

\section{$4.14 \mathrm{H}-\mathrm{He}-\mathrm{H}^{2+}$ LT}

Geometries as in the $\mathrm{H}-\mathrm{He}-\mathrm{H}$ calculations.

EIGENVECTORS

$\begin{array}{ccccc}1 & 2 & 3 & 4 & 5 \\ -2.4415 & -1.0292 & -0.8944 & -0.2216 & -0.1515 \\ \text { AG } & \text { AG } & \text { B1U } & \text { AG } & \text { B1U }\end{array}$

NATURAL ORBITALS IN ATOMIC ORBITAL BASIS 


$\begin{array}{ccccc}1 & 2 & 3 & 4 & 5 \\ 0.9987 & 0.9987 & 0.0012 & 0.0012 & 0.0000 \\ A G & B 1 U & A G & B 1 U & B 2 G\end{array}$

ENERGY COMPONENTS

$\begin{array}{rr}\text { WAVEFUNCTION NORMALIZATION }= & 1.0000000000 \\ & \\ \text { ONE ELECTRON ENERGY }= & -4.3879612485 \\ \text { TWO ELECTRON ENERGY }= & 0.4199226032 \\ \text { NUCLEAR REPULSION ENERGY }= & 1.8918264891 \\ \text { TOTAL ENERGY }= & -2.0762121561\end{array}$

ELECTRON-ELECTRON POTENTIAL ENERGY =

0.4199226032 NUCLEUS-ELECTRON POTENTIAL ENERGY =

$-6.9931707086$ NUCLEUS-NUCLEUS POTENTIAL ENERGY =

1.8918264891

TOTAL POTENTIAL ENERGY =

$-4.6814216163$

TOTAL KINETIC ENERGY =

2.6052094601

VIRIAL RATIO $(\mathrm{V} / \mathrm{T})=$

1.7969463446

COEFFICIENT/ OCCUPIED ACTIVE SPIN ORBITALS

$\begin{array}{rrr}0.7068995307 \mathrm{E}+00 & 2 & -1 \\ -0.7068995307 \mathrm{E}+00 & 1 & -2 \\ 0.1461272377 \mathrm{E}-01 & 4 & -3 \\ -0.1461272377 \mathrm{E}-01 & 3 & -4\end{array}$

Atomic Contributions for neq: 1

Atom number

: 1

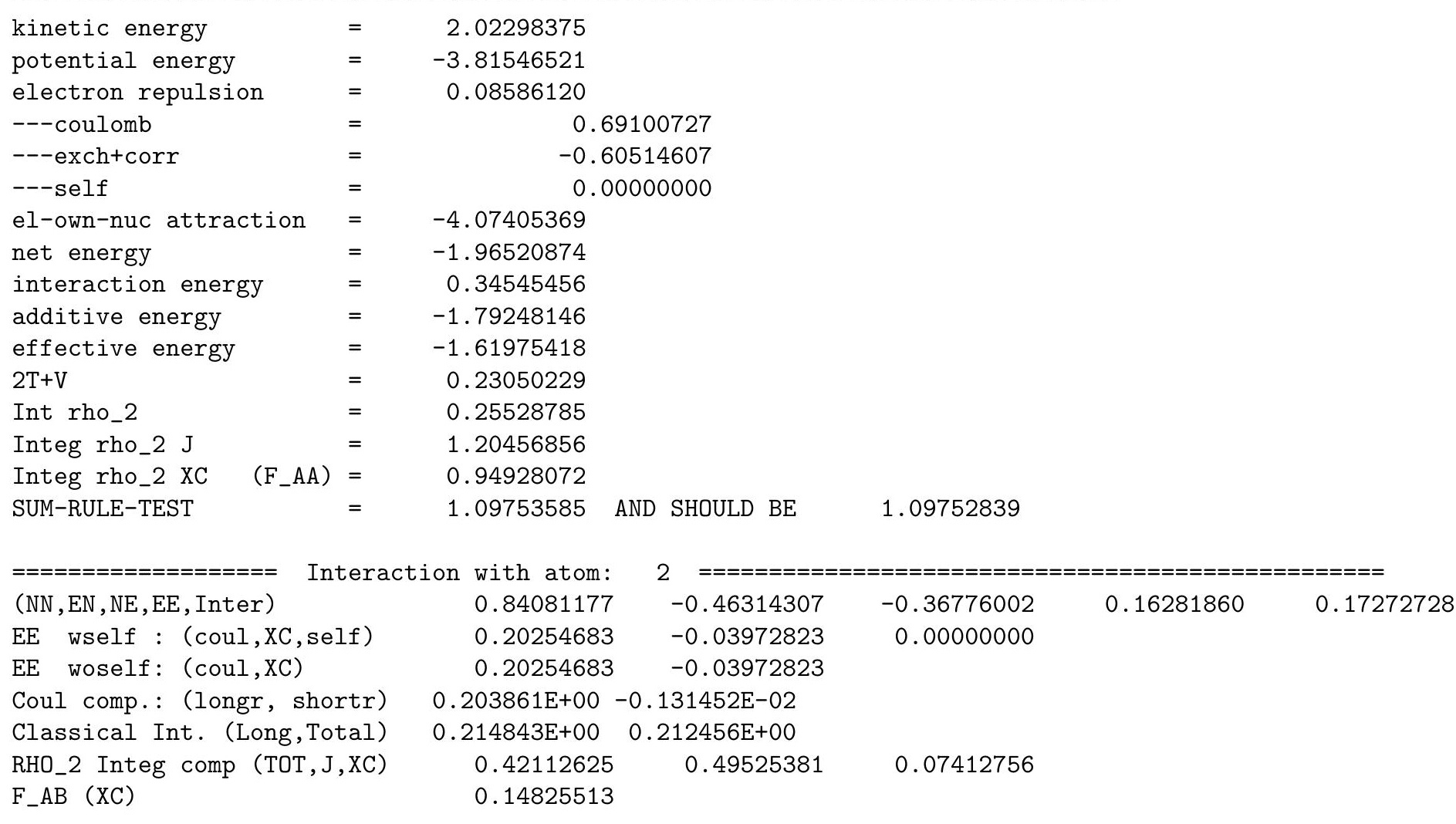




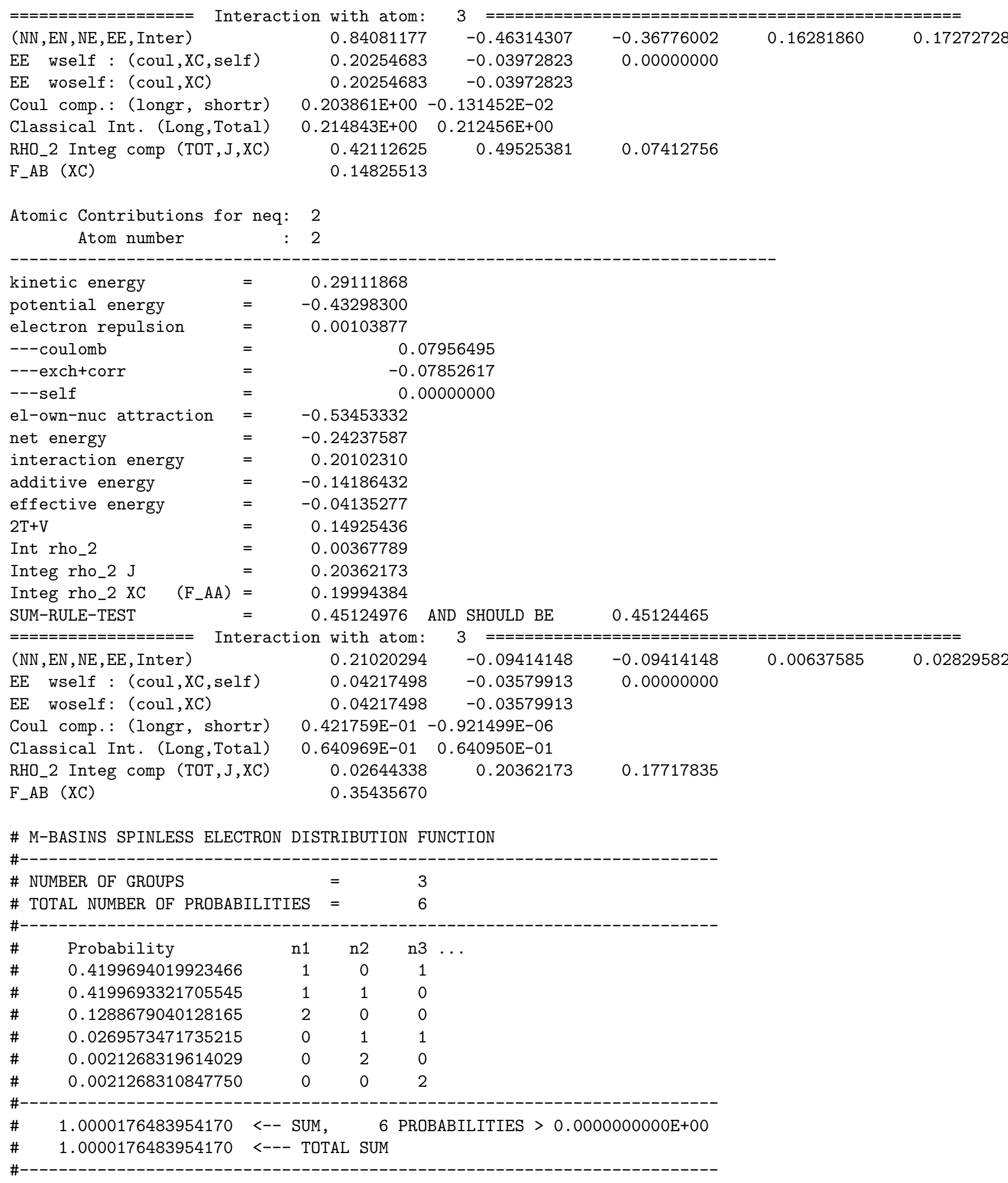

Average populations and localization indices

$\begin{array}{lll}\#<\mathrm{n}(1)> & = & 1.0976745422 \\ \#<\mathrm{n}(2)> & = & 0.4511803433 \\ \#<\mathrm{n}(3)> & = & 0.4511804113 \\ \#<\mathrm{n}(2) \mathrm{n}(1)> & = & 0.4199693322\end{array}$




\begin{tabular}{|c|c|c|c|c|c|c|}
\hline$\#<\mathrm{n}(3)$ & $\mathrm{n}($ & 1) $>$ & $=$ & 0.4199694020 & & \\
\hline$\#<n\left(\begin{array}{ll}3\end{array}\right)$ & $\mathrm{n}($ & 2) $>$ & $=$ & 0.0269573472 & & \\
\hline$\#<\mathrm{n}($ & $\mathrm{n}($ & 2) $n(1)>$ & $=$ & 0.0000000000 & & \\
\hline lta_ & 1 & 1) & $=$ & 0.9471535925 & $\%$ Localization = & 86.2873 \\
\hline $1 \mathrm{ta} \mathbf{s}_{-}$ & 2 & 2) & $=$ & 0.1993100382 & $\%$ Localization = & 44.1752 \\
\hline lta_ & 3 & 3) & $=$ & 0.1993101014 & $\%$ Localization = & 44.1753 \\
\hline
\end{tabular}

Delocalization indices, Eq. (28) J. Chem. Phys. 126, 094102 (2007)

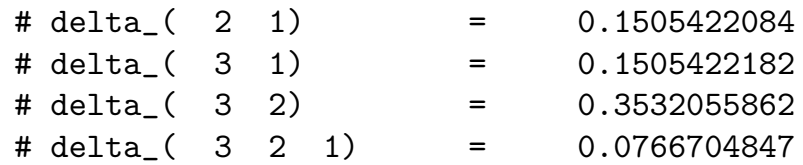

\# ALLSPINS command has been read in from the input file

\#

\# Fragment A formed by atoms 1

\# Fragment B formed by atoms 23

\begin{tabular}{|c|c|c|c|c|}
\hline \# & $\left\langle\mathrm{S}_{-} \mathrm{A}^{\wedge} 2\right\rangle=$ & 0.88696825 , & $<\mathrm{S}_{-} \mathrm{AB}^{\wedge} 2>=$ & 0.21056312 \\
\hline & $\operatorname{rho}^{-1}(r 1 ; r 1)$ & 0.82314629 & rho^1(r1;r1) & 0.00000000 \\
\hline$\#$ & $r h o^{\wedge} 2(r 1, r 2 ; r 1, r 2)$ part $=$ & -0.06382196 & rho`2(r1,r2;r1,r2) part $=$ & -0.21056312 \\
\hline \# & $r h o^{\wedge} 2(r 1, r 2 ; r 2, r 1)$ part $=$ & 0.12764392 , & $r h o^{\wedge} 2(r 1, r 2 ; r 2, r 1)$ part $=$ & 0.42112625 \\
\hline \# & $\left\langle\mathrm{S}_{-} \mathrm{B}^{\wedge} 2\right\rangle=$ & 0.69192761 & & \\
\hline & $r h o^{\wedge} 1(r 1 ; r 1)$ & 0.67686697 & & \\
\hline & $r h o^{\wedge} 2(r 1, r 2 ; r 1, r 2)$ part $=$ & -0.01506064 & & \\
\hline & $r h o^{\wedge} 2(r 1, r 2 ; r 2, r 1)$ part $=$ & 0.03012127 & & \\
\hline
\end{tabular}

\# Fragment A formed by atoms 2

\# Fragment B formed by atoms 13

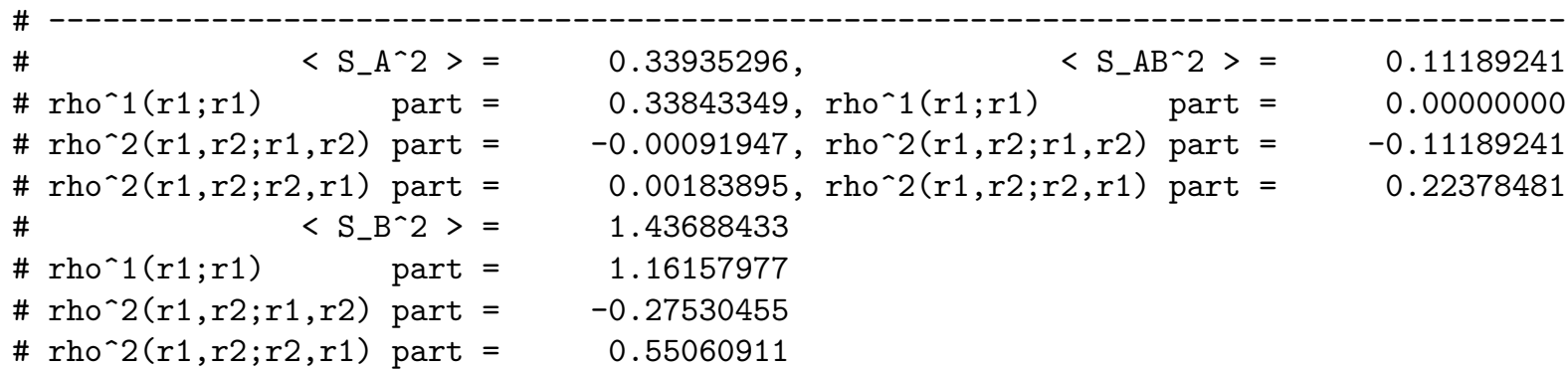

\section{$4.15 \mathrm{H}-\mathrm{He}^{-\mathrm{H}^{2+}} \mathrm{BS}$}

Geometries as in the $\mathrm{H}-\mathrm{He}-\mathrm{H}$ calculations. Real space calculations unnecessary.

EIGENVECTORS

$\begin{array}{ccccc}1 & 2 & 3 & 4 & 5 \\ -1.7988 & -0.7232 & -0.7040 & -0.2183 & -0.1574 \\ \text { A1 } & \text { A1 } & \text { B2 } & \text { A1 } & \text { B2 }\end{array}$

NATURAL ORBITALS IN ATOMIC ORBITAL BASIS

$\begin{array}{ccccc}1 & 2 & 3 & 4 & 5 \\ 1.9796 & 0.0135 & 0.0028 & 0.0020 & 0.0019 \\ \mathrm{~A} 1 & \mathrm{~A} 1 & \mathrm{~B} 2 & \mathrm{~B} 1 & \mathrm{~A} 1\end{array}$

ENERGY COMPONENTS 


$\begin{array}{rr}\text { WAVEFUNCTION NORMALIZATION }= & 1.0000000000 \\ \text { ONE ELECTRON ENERGY }= & -5.5327048134 \\ \text { TWO ELECTRON ENERGY }= & 0.9123610411 \\ \text { NUCLEAR REPULSION ENERGY }= & 1.9756107487 \\ \text { TOTAL ENERGY }= & -2.6447330237 \\ & \\ \text { N-ELECTRON POTENTIAL ENERGY }= & 0.9123610411 \\ \text {-ELECTRON POTENTIAL ENERGY }= & -8.3154471191 \\ \text { TS-NUCLEUS POTENTIAL ENERGY }= & 1.9756107487 \\ \text { TOTAL POTENTIAL ENERGY }= & -5.4274753293 \\ \text { TOTAL KINETIC ENERGY }= & 2.7827423057 \\ \text { VIRIAL RATIO }(\mathrm{V} / \mathrm{T}) & =\end{array}$

\section{$4.16 \mathrm{H}-\mathrm{He}-\mathrm{H}^{2+}$ BT}

Geometries as in the $\mathrm{H}-\mathrm{He}-\mathrm{H}$ calculations. Real space calculations unnecessary.

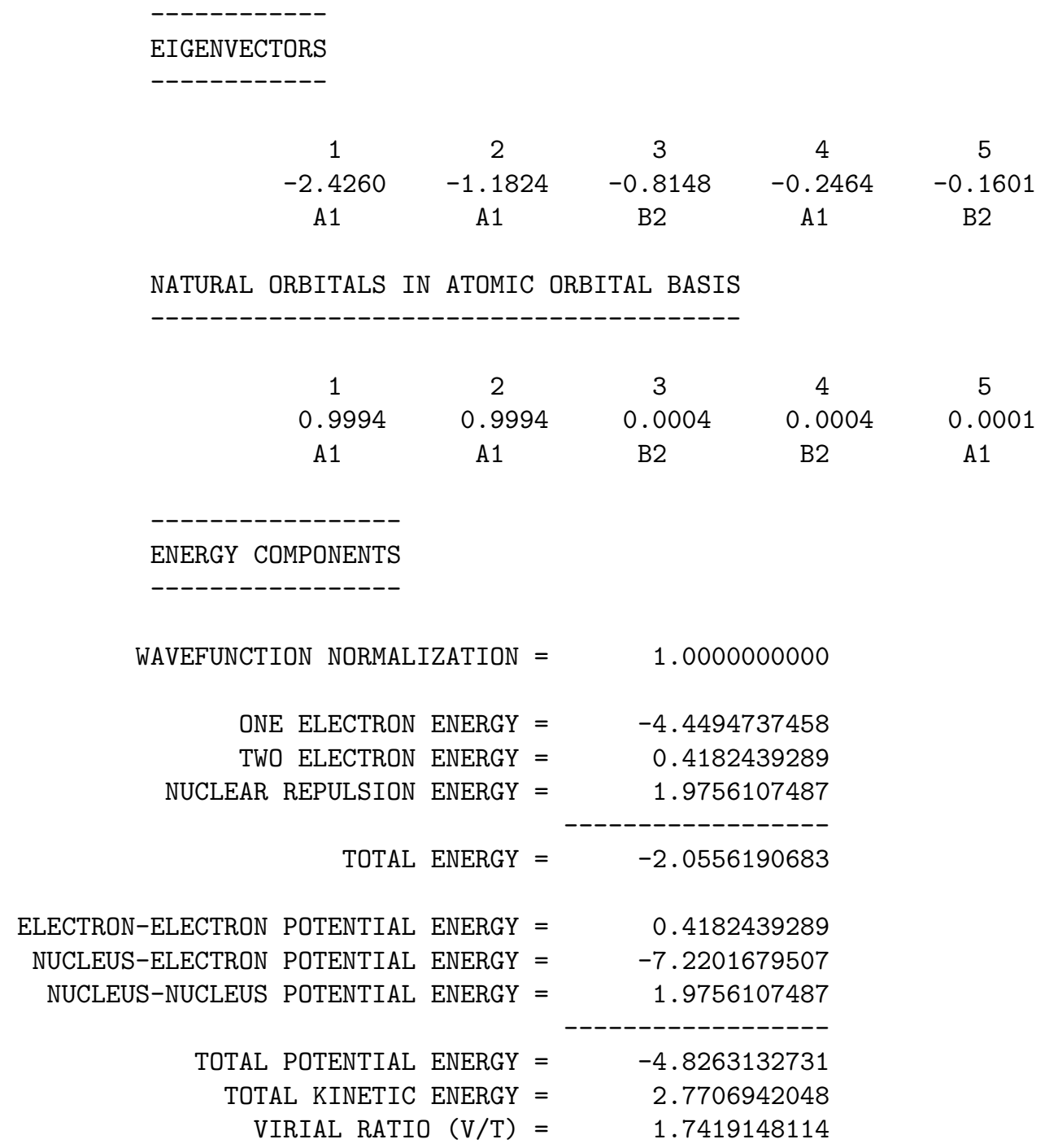




\subsection{Li-Be-Li}

CAS $[7,4] / / 6-311+\mathrm{G}^{* *}$ calculations. $R_{\mathrm{LiBe}}=8.00$ au. All energies in au and angles in degrees.

1. Singlet, triplet energies in $\mathrm{Li}_{2}$

$$
\begin{aligned}
& \text { Angle ESINGLET ETRIPLET } \\
& 180-14.8640162915-14.8640089201 \\
& 170-14.8640166654-14.8640088186 \\
& 160-14.8640179265-14.8640084736 \\
& 150-14.8640205861-14.8640077380 \\
& 140-14.8640258553-14.8640062613 \\
& 130-14.8640365022-14.8640032417 \\
& 120-14.8640590040-14.8639968285 \\
& 115-14.8640789469-14.8639911628 \\
& 110-14.8641090079-14.8639826924 \\
& 100-14.8642253790-14.8639507350 \\
& 95-14.8643348534-14.8639216702 \\
& 90-14.8645056749-14.8638777391 \\
& 85-14.8647728338-14.8638115238 \\
& 80-14.8651897879-14.8637122200 \\
& 75-14.8658351782-14.8635641753 \\
& 70-14.8668172157-14.8633442042 \\
& 65-14.8682675873-14.8630154128 \\
& 60-14.8703092363-14.8625118139 \\
& 50-14.8760687111-14.8603078125 \\
& 40-14.8800768745-14.8528858297 \\
& 30-14.8632556745-14.8247078821 \\
& 20-14.7693660110-14.7275683186 \\
& 10-14.2257515608-14.1595838563
\end{aligned}
$$

2. Singlet, triplet energies in Li-Be-Li

$$
\begin{aligned}
& \text { Angle ESINGLET ETRIPLET } \\
& 180-29.4355480285-29.4350697655 \\
& 170-29.4355376664-29.4350712209 \\
& 160-29.4355066397-29.4350759487 \\
& 150-29.4354550889-29.4350850004 \\
& 140-29.4353831903-29.4351000235 \\
& 130-29.4352913819-29.4351230707 \\
& 120-29.4351816115-29.4351561443 \\
& 115-29.4351219943-29.4351767859 \\
& 110-29.4350619088-29.4351999971 \\
& 105-29.4350053140-29.4352252089 \\
& 100-29.4349593395-29.4352512476 \\
& 95-29.4349363814-29.4352760258 \\
& 90-29.4349575404-29.4352961360 \\
& 85-29.4350578987-29.4353063276 \\
& 80-29.4352940327-29.4352987915 \\
& 75-29.4357532099-29.4352619843 \\
& 70-29.4365608029-29.4351782397 \\
& \begin{array}{llll}
60 & -29.4398572061 & -29.4347285476
\end{array}
\end{aligned}
$$

3. Densities at the bond critical point $\rho_{b c p}$ in the singlet and triplet Li-Be-Li
Angle rhob s rhob t

$180 \quad 0.238910258 \mathrm{E}-02 \quad 0.230549032 \mathrm{E}-02$




$\begin{array}{rrr}170 & 0.238719165 \mathrm{E}-02 & 0.230530755 \mathrm{E}-02 \\ 160 & 0.238149998 \mathrm{E}-02 & 0.230487429 \mathrm{E}-02 \\ 150 & 0.237233652 \mathrm{E}-02 & 0.230450644 \mathrm{E}-02 \\ 140 & 0.236008275 \mathrm{E}-02 & 0.230462035 \mathrm{E}-02 \\ 130 & 0.234524516 \mathrm{E}-02 & 0.230563773 \mathrm{E}-02 \\ 120 & 0.232834751 \mathrm{E}-02 & 0.230784711 \mathrm{E}-02 \\ 115 & 0.231930863 \mathrm{E}-02 & 0.230944821 \mathrm{E}-02 \\ 110 & 0.231011185 \mathrm{E}-02 & 0.231138336 \mathrm{E}-02 \\ 105 & 0.230100513 \mathrm{E}-02 & 0.231365771 \mathrm{E}-02 \\ 100 & 0.229237808 \mathrm{E}-02 & 0.231626382 \mathrm{E}-02 \\ 95 & 0.228492857 \mathrm{E}-02 & 0.231920130 \mathrm{E}-02 \\ 90 & 0.227973264 \mathrm{E}-02 & 0.232244225 \mathrm{E}-02 \\ 85 & 0.227857929 \mathrm{E}-02 & 0.232593967 \mathrm{E}-02 \\ 80 & 0.228437179 \mathrm{E}-02 & 0.232960161 \mathrm{E}-02 \\ 75 & 0.230181108 \mathrm{E}-02 & 0.233329603 \mathrm{E}-02 \\ 70 & 0.233864717 \mathrm{E}-02 & 0.233692547 \mathrm{E}-02 \\ 60 & 0.252963063 \mathrm{E}-02 & 0.234534634 \mathrm{E}-02\end{array}$




\section{References}

[1] R. F. W. Bader, Atoms in Molecules, Oxford University Press, Oxford, 1990.

[2] A. D. Becke and K. E. Edgecombe, J. Chem. Phys., 1990, 92, 5397.

[3] M. A. Blanco, A. Martín Pendás and E. Francisco, Journal of Chemical Theory and Computation, 2005, 1, 1096-1109.

[4] D. Menéndez-Crespo, A. Costales, E. Francisco and A. Martín Pendás, Chem. Eur. J., 2018, 26, 9101-9112.

[5] E. Francisco, A. Martín Pendás and M. A. Blanco, J. Chem. Phys., 2007, 126, 094102-1-094102-13.

[6] A. Martín Pendás, E. Francisco and M. A. Blanco, J. Chem. Phys., 2007, 127, 144103.

[7] C. Outeiral, M. A. Vincent, A. Martín Pendás and P. L. A. Popelier, Chem. Sci., 2018, 9, 5517-5529.

[8] E. Francisco, A. Martín Pendás, M. García-Revilla and R. Álvarez Boto, Comput. Theor. Chem., 2013, 1003, 71-78.

[9] A. Martín Pendás, E. Francisco and M. A. Blanco, Phys. Chem. Chem. Phys., 2007, 9, 1087-1092.

[10] A. Martín Pendás and E. Francisco, Phys. Chem. Chem. Phys., 2018, 20, 16231-16237.

[11] A. E. Clark and E. R. Davidson, The Journal of Chemical Physics, 2001, 115, 7382-7392.

[12] A. Martín Pendás and E. Francisco, Physical Chemistry Chemical Physics, 2021, 23, 8375-8392.

[13] M. W. Schmidt, K. K. Baldridge, J. A. Boatz, S. T. Elbert, M. S. Gordon, J. H. Jensen, S. Koseki, N. Matsunaga, K. A. Nguyen, S. J. Su, T. L. Windus, M. Dupuis and J. A. Montgomery, J. Comput. Chem., 1993, 14, 1347.

[14] L. R. K. P. Baybutt and D. G. Truhlar, J. Chem. Phys., 1976, 65, 3826. 